\title{
Wirtschafts- bzw. Unternehmensethik: Eine Analyse des Forschungsstandes
}

„Bei der Unterschiedlichkeit des Anspruchs, der in der Philosophie und der $B W L$ an die Ethik gestellt wird, und der Vielfalt ethischer Positionen ist es nicht verwunderlich, dass in der BWL verschiedenartige und kontroverse Haltungen gegenüber der Unternehmensethik bestehen. Die intensivere Befassung mit Fragen der Unternehmensethik seit ca. 1985 hat zur Entwicklung mehrerer unternehmensethischer Ansätze geführt, in denen sich unterschiedliche Grundkonzeptionen der Ethik und Konzepte zur Begründung von Werten sowie Normen niederschlagen. "1

Nachdem nun im vorigen Kapitel die Relevanz einer reflektierten Beschäftigung mit wirtschaftsethischen Fragen für die Betriebswirtschaftslehre als praktischnormative Disziplin erarbeitet wurde, befasst sich die Arbeit im Folgenden mit dem aktuellen Forschungsstand der Wirtschafts- und insbesondere der Unternehmensethik. Nach einer terminologischen Präzisierung einiger zentraler Begriffe erfolgt eine Systematisierung der Forschungsrichtungen der Ethik sowie die Einordnung der Wirtschaftsethik als Teildisziplin der angewandten Ethik. Darüber hinaus wird das klassische Dreiebenenmodell der Wirtschaftsethik präsentiert, in welchem die Unternehmensethik auf der Mesoebene verankert wird. Hieran anschließend wird der Forschungsstand im Bereich der Wirtschafts- bzw. Unternehmensethik vorgestellt, wobei gerade die umfangreich ausgearbeiteten Konzeptionen des deutschsprachigen Raums, welche zur Bildung verschiedener Wirtschaftsethikschulen geführt haben, detailliert eruiert werden. Des Weiteren wird auch die konzeptionell deutlich pragmatischer ausgerichtete sowie praxisorientiertere Forschung im angelsächsischen Raum erörtert und der Forschung des deutschsprachigen Raums gegenübergestellt. Nach einer kritischen Reflexion des gegenwärtigen Forschungsstands wird die Notwendigkeit der Entwicklung einer neuen, genuinen Mesoethik auf systemtheoretischer Basis verdeutlicht.

\footnotetext{
${ }^{1}$ Küpper, H.-U. (2011), S. 107.

(C) Der/die Autor(en) 2022

F. Fuchs, Subjektunabhängige, analytische Unternehmensethik, https://doi.org/10.1007/978-3-658-36480-9_3
} 


\subsection{Begriffliche Grundlagen}

Die Termini Ethik, Moral und Ethos stellen zweifellos zentrale Begrifflichkeiten der Ethikforschung dar. Während hinsichtlich der Substantive noch ein weitestgehender Konsens im wissenschaftlichen Kontext, aber auch meist in der alltagssprachlichen Verwendung besteht, werden gerade die verwandten Adjektive „ethisch“ und ,moralisch“ vielfach synonym und undifferenziert gebraucht ${ }^{2}$ - insbesondere im englischsprachigen Raum. ${ }^{3}$ Aufgrund der Relevanz dieser Termini sowie zu deren konzeptionellen Präzisierung werden diese Zentralbegrifflichkeiten daher im Folgenden näher erörtert.

\subsubsection{Ethik, Moral und Ethos}

Ihren historischen Ursprung finden die genannten Begrifflichkeiten in der klassischen Antike. Der Begriff der Ethik lässt sich hierbei auf den griechischen

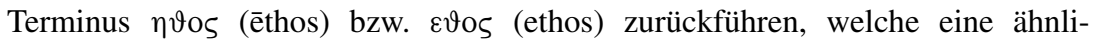
che, aber nicht deckungsgleiche Bedeutung aufweisen. Der Begriff des $\eta \vartheta$ bezeichnete dabei ursprünglich den gewöhnlichen bzw. natürlichen Wohn- und Aufenthaltsort ${ }^{4}$ eines Lebewesens. ${ }^{5}$ Im Laufe der Zeit verschob sich der Begriffsinhalt hin zu verinnerlichten Normen im Sinne von „Gewohnheit“, „Sitte“ bzw. „Brauch“, wobei ebenso im starken Maße ein tugendhafter Charakter hervorgehoben werden kann. Bedeutsam ist bereits hier, dass $\eta \vartheta ૦ \varsigma$ sowohl im neutralen,

\footnotetext{
${ }^{2}$ NoLL spricht in diesem Zusammenhang sogar von einem „Begriffswirrwarr“ (Noll, B. (2013), S. 27). Vgl. hierzu auch die Überlegungen in Holzmann, R. (2019), S. 3; Reichelt, M. (2018), S. 17; Küpper, H.-U. (2016), S. 15; Küpper, H.-U. (2015), S. 323; Oermann, N. O. (2015), S. 11; Noll, B. (2013), S. 27; Hentze, J. / Thies, B. (2012), S. 10; Clausen, A. (2009), S. 27; Horster, D. (2009), S. 7; Lenk, H. (1997), S. 6; Steinmann, H. / Löhr, A. (1994a), S. 8; Frankena, W. K. (1973), S. 5-6.

${ }^{3}$ So wird z. B. auch in der englischsprachigen Literatur häufig ,immoral“ und ,unethical“ undifferenziert verwendet. Vgl. z. B. Carroll, A. B. (2000), S. 37-38, welcher die Begriffe synonym gebraucht. Vgl. auch Waluchow, W. J. (2003), S. 13-14; Frankena, W. K. (1973), S. 6.

${ }^{4}$ ROMMERSKIRCHEN verweist hier beispielhaft auf den etymologischen Ursprung des Begriffs, der sich auf eine Hirtenwiese bezog, auf welcher die Menschen ihr Handeln nachhaltig konsensualistisch ausrichten müssen, da die Ressourcen beschränkt sind und daher ein funktionsfähiges Ethos zur Konfliktvermeidung notwendig ist. Vgl. Rommerskirchen, J. (2019), S. 17.

5 Vgl. Rommerskirchen, J. (2019), S. 17; Höffe, O. (2018), S. 9; Charpenel, E. (2017), S. 28; Andersen, S. (2005), S. 1.
} 
klassifikatorischen Sinne, d. h. als Zuordnung eines Gegenstandes zum Gebiet der Ethik zugehörig, als auch in einem wertenden Sinne gebraucht werden kann. ${ }^{6}$ Eine ähnliche Bedeutung weist gleichfalls der verwandte Begriff des $\varepsilon \vartheta \circ \varsigma$ auf, welcher ebenso Sitten und Gebräuche bezeichnet, jedoch enger auszulegen ist, da hier eine sehr viel stärkere Konnotation hinsichtlich der Externalität der Moralvorstellungen existiert ${ }^{7}$, während $\eta \vartheta ० \varsigma$ auch eine Verinnerlichung der Normen impliziert. Der Begriff der Moral geht wiederum auf die lateinische Übersetzung der griechischen Termini in ,,mos" zurück $^{8}$ und bezeichnet ähnlich wie bei beiden zuvor genannten Begriffen die gebräuchlichen Sitten an einem Ort. ${ }^{9}$ Aus der aufgezeigten etymologischen Entwicklung ist die enge Verbindung der Terminologien evident, so dass die alltagssprachlich teilweise etwas undifferenzierte Begriffsverwendung, gerade im adjektivischen Gebrauch, historisch durchaus gerechtfertigt werden könnte.

Allerdings ist festzustellen, dass, bereits aus Präzisionsgründen, in der fachsprachlichen Verwendung hinsichtlich der Begriffe Ethik, Moral sowie Ethos ein klarer terminologischer Konsens besteht. Unter Moral können in diesem Sinne die in einer gewissen Gesellschaft verbreiteten Normvorstellungen über die Frage, was ,als Handlung, Zustand oder Haltung für gut und wünschenswert $[\ldots]$ gehalten wird“"10 aufgefasst werden, wobei diese Normen unbedingte

\footnotetext{
${ }^{6}$ Vgl. Hübner, D. (2018), S. 11. Siehe hierzu auch die Überlegungen zur Differenzierung zwischen „ethisch“ und „moralisch“ im nachfolgenden Unterkapitel 3.1.2.

${ }^{7}$ Der Begriff des $\varepsilon \vartheta ૦ \varsigma$ könnte daher im Sinne der heutigen Begriffsauffassung von „Moral“ gedeutet werden, während im Begriff des $\eta \vartheta ૦ \varsigma$ durch die Verinnerlichung von Normen der moderne Begriff des „Ethos“ enthalten ist. Vgl. hierzu auch PIEPER, welche betont: „Das $\varepsilon \vartheta ० \varsigma$ wird dann zum $\eta \vartheta \circ \varsigma$ im Sinne von Charakter; es verfestigt sich zur Grundhaltung der Tugend.“ (Pieper, A. (2017), S. 22). Vgl. ähnlich auch Fenner, D. (2020), S. 16.

${ }^{8}$ Die erste Übersetzung wird vielfach CICERO zugeschrieben. Vgl. Conrad, C. A. (2020), S. 11; Rommerskirchen, J. (2019), S. 19; Härle, W. (2018b), S. 9; Wahler, H. (2018), S. 7; Burkhardt, H. (2017), S. 14; Ricken, F. (2013), S. 16.

${ }^{9}$ Vgl. Fenner, D. (2020), S. 16-17; Rommerskirchen, J. (2019), S. 17; Härle, W. (2018b), S. 8-9; Höffe, O. (2018), S. 10; Hübner, D. (2018), S. 11-12; Veith, W. (2018), S. 4; Wahler, H. (2018), S. 7-8; Burkhardt, H. (2017), S. 12-14; Pieper, A. (2017), S. 21-22; Ricken, F. (2013), S. 16; Heidenreich, F. (2012), S. 18; Hepfer, K. (2008), S. 14; Schrey, H.-H. (1991), S. 15-16; Wundt, W. (1912), S. 20-22.

${ }^{10}$ Göbel, E. (2020), S. 31; Göbel, E. (2017), S. 26. Vgl. ähnlich auch Arnold, D. G. / Beauchamp, T. L. / Bowie, N. E. (2020), S. 1; Bak, P. M. (2014), S. 2.
} 
Geltung beanspruchen. ${ }^{11}$ Solche Normvorstellungen sind folglich überindividuell und können damit im Sinne des „overlapping consensus“12 nach RAWLS ${ }^{13}$ als gemeinsam geteilte Wertvorstellungen ${ }^{14}$ begriffen werden. Das Ethos ist hierbei in heutiger Terminologie wesentlich enger als die Moral aufzufassen und bezeichnet die individuell verinnerlichten moralischen Vorstellungen. ${ }^{15}$ Dabei stehen Moral und Ethos in steter Interaktion. So beeinflussen gesellschaftliche Moralvorstellungen ebenso das Ethos von Individuen, wie auch vice versa die persönliche Haltung von Menschen auf Dauer die Moral der Gesellschaft verändern kann. Diesen Zusammenhang visualisiert auch nochmals die nachfolgende Abbildung 3.1.

\section{Abbildung 3.1}

Zusammenhang zwischen Moral als äußere Normen und Ethos als verinnerlichte Normen ${ }^{16}$

\section{Internalisierung}

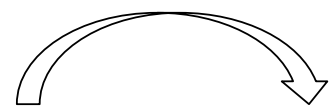

Äußere Normen

(Moral)

Innere Normen (Ethos)

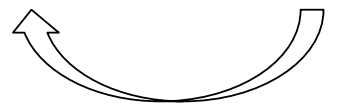

Veränderung

${ }^{11}$ Vgl. Hübner, D. (2018), S. 13. Vgl. hierzu auch Birnbacher, D. (2013), S. 13; Rehbock, T. (2011), S. 233; Solomon, R. C. (2010), S. 18.

12 Eine spannende Überlegung ergibt sich hierbei in der Frage, wie weit dieser ,overlapping consensus" reicht, welche im Rahmen dieser Arbeit insbesondere vor dem Hintergrund der Überlegungen zum Weltethos und zur Common Morality Theory wieder aufgegriffen wird. Vgl. hierzu Abschnitt 4.5.2.

${ }^{13}$ Vgl. hierzu Rawls, J. (2005), S. 133-172; Rawls, J. (1999), S. 340; Rawls, J. (1987), S. 1. ${ }^{14}$ Zur Diskussion des Wertbegriffs sowie einer Abgrenzung des ethischen zum ökonomischen Wertbegriff vgl. Dietzfelbinger, D. (2015), S. 45 sowie Hartmann, N. (1962), S. 250253.

${ }^{15}$ Vgl. Conrad, C. A. (2020), S. 12; Göbel, E. (2020), S. 34; Bak, P. M. (2014), S. 4.

${ }^{16}$ In Anlehnung an Göbel, E. (2020), S. 35. Äußere Normen können auch rechtlicher Natur sein, hiervon sei jedoch im Rahmen dieser Ausführungen abstrahiert. 
In Abgrenzung hierzu wird in der Literatur Ethik vielfach als die „Wissenschaft von der Moral ${ }^{* 17}$, d. h. die systematische bzw. kritische Reflexion ${ }^{18}$ über die Moral als Untersuchungsgegenstand ${ }^{19}$, aufgefasst. ${ }^{20}$ Aus diesem Grunde wird die Ethik vielfach auch als Philosophie der Moral $^{21}$ bzw. als Moralphilosophie bezeichnet. ${ }^{22}$ Wie die vorigen Ausführungen jedoch zeigen, erscheint eine begriffliche Verengung auf die Moral als Forschungsgegenstand allerdings noch zu kurz zu greifen, da hierbei persönliche Überzeugungen (Ethos) ausgeklammert blieben. Daher folgt die Arbeit der Auffassung GöBELS, dass als Ethik die systematische Reflexion von Moral und Ethos, d. h. normativen Werthaltungen auf kollektiver wie individueller Ebene ${ }^{23}$, aufzufassen ist. ${ }^{24}$ Die Ethik befasst sich folglich mit einem Metadiskurs, in welchem individuelle wie kollektive Moralvorstellungen Gegenstand der wissenschaftlichen Analyse bzw. Begründung sind. In diesem Sinne kann Ethik nach LINGNAU als „,die Lehre der Rechtfertigung von

${ }^{17}$ Hübner, D. (2018), S. 17, in der Quelle ebenfalls herv.

18 Vgl. Andersen, S. (2005), S. 1.

${ }^{19}$ Eine solche Beziehung findet sich auch beispielsweise bei der Ökonomik wieder, welche als Untersuchungsgegenstand die Ökonomie aufweist oder in der Psychologie in Bezug auf die Psyche.

${ }^{20}$ Vgl. auch Höffe, O. (2018), S. 10-11; Pieper, A. (2017), S. 24; Birnbacher, D. (2013), S. 2; Vieth, A. (2006), S. 12. Eine solche Differenzierung findet z. T. im angelsächsischen Bereich nicht statt (so z. B. bei Ferrell, O. C. / Fraedrich, J. / Ferrell, L. (2017), S. 5; Shaw, W. H. (2017), S. 4; Solomon, R. C. (2010), S. 12-13). Vgl. hierzu auch Hübner, D. (2018), S. 20. Es existieren jedoch auch einige englischsprachige Quellen, welche eine ähnliche Systematisierung vornehmen, vgl. z. B. Arnold, D. G. / Beauchamp, T. L. / Bowie, N. E. (2020), S. 1; Crane, A. et al. (2019), S. 8; Velasquez, M. G. (2014), S. 8 und S. 13; Frankena, W. K. (1973), S. 4-5.

${ }^{21}$ Dem eigenen Selbstverständnis nach ist die Philosophie Wissenschaft, da sie systematisch und nach Vernunftgründen vorgeht. Vgl. hierzu Schöndorf, H. (2010a), S. 360.

${ }^{22}$ Vgl. Arnold, D. G. / Beauchamp, T. L. / Bowie, N. E. (2020), S. 1; Fenner, D. (2020), S. 19 und S. 23; Gerhardt, V. (2018), S. 100; Reichelt, M. (2018), S. 17; Shaw, W. H. (2017), S. 3; Birnbacher, D. (2013), S. 2; Ricken, F. (2013), S. 18; Hentze, J. / Thies, B. (2012), S. 13; Rehbock, T. (2011), S. 235; Dutt, A. K. / Wilber, C. K. (2010), S. 4; Celikates, R. / Gosepath, S. (2009), S. 7; Knoepffler, N. (2006), S. 10; Schweppenhäuser, G. (2006), S. 15; Fisher, J. (2004), S. 398; Lenk, H. (1997), S. 6; Steinmann, H. / Löhr, A. (1994a), S. 8 und S. 11; Rothschild, K. W. (1992), S. 2; Hoerster, N. (1982), S. 9; Frankena, W. K. (1973), S. 4; Cathrein, V. (1911), S. 1.

${ }^{23}$ PAUER-STUDER verwendet in diesem Sinne analog zur Abgrenzung von Ethos und Moral auch die Differenzierung zwischen „Individual-“ und „Sozialmoral“ (vgl. Pauer-Studer, H. (2020), S. 16-18).

${ }^{24}$ Vgl. Göbel, E. (2020), S. 36. 
Normen, d. h. das methodisch disziplinierte Nachdenken darüber, welche Normen zur Begründung bzw. Rechtfertigung von Handlungen herangezogen werden sollen“25 definiert werden. Ähnlich, wenngleich auf die Moral als Objekt der Ethik fokussiert, begreift auch FRANKENA ,ethics as philosophy that is concerned with morality and its problems and judgments, or with moral problems and judgments. “26

Die Ethik gehört nach der Klassifikation des ARISTOTELES ${ }^{27}$ zur praktischen Philosophie ${ }^{28}$, welche sich in Abgrenzung zur theoretischen Philosophie ${ }^{29}$, die auf Wissen als reine Erkenntnis abzielt, mit dem Handeln des Menschen (d. h. der menschlichen Praxis) befasst. ${ }^{30}$ Die Begriffsverwendung des Terminus „Ethik“ lässt sich dabei bis zu SOKRATES zurückverfolgen ${ }^{31}$, welcher ihn im Kontext der Untersuchung von guten und gerechten Handlungen verwendete. ${ }^{32}$ Als Begründer einer eigenständigen akademischen Disziplin, die mittlerweile eine fast 2500-jährige Geschichte aufweist ${ }^{33}$, gilt dagegen ARISTOTELES ${ }^{34}$, der mit seiner Nikomachischen Ethik ${ }^{35}$ eine bedeutende Tugendlehre konzipierte.

\footnotetext{
${ }^{25}$ Lingnau, V. / Schäffer, U. (2009), S. 284. Vgl. ähnlich auch Holzmann, R. (2019), S. 6; Hepfer, K. (2008), S. 10; Löhr, A. (1996), S. 53; Steinmann, H. / Löhr, A. (1994a), S. 8.

${ }^{26}$ Frankena, W. K. (1973), S. 5.

${ }^{27}$ Vgl. Aristoteles (1989), Buch VI, Kapitel 1. Vgl. zur Übersicht Höffe, O. (2014), S. 32-33. ${ }^{28}$ Hierzu gehören auch die Ökonomik sowie die Politik. Vgl. Göbel, E. (2020), S. 71; Höffe, O. (2014), S. 34; Rohls, J. (1999), S. 1.

${ }^{29}$ Beispiele hierzu sind die Logik, Physik, Mathematik, Metaphysik sowie die Theologie. Vgl. Pieper, A. (2017), S. 21; Höffe, O. (2014), S. 31 und S. 34.

${ }^{30}$ Vgl. Höffe, O. (2008b), S. 9-10.

${ }^{31}$ Zur Einführung in die historischen Grundlagen der Ethik vgl. die Ausführungen bei Attfield, R. (2012), S. 1-35; Düwell, M. / Hübenthal, C. / Werner, M. H. (2011), S. 3-11 sowie umfassend Rohls, J. (1999); MacIntyre, A. C. (1998); Wundt, M. (1985); Köstlin, K. R. (1975).

${ }^{32}$ Vgl. Rommerskirchen, J. (2019), S. 16.

${ }^{33}$ Vgl. Arnold, D. G. / Beauchamp, T. L. / Bowie, N. E. (2020), S. 33.

${ }^{34}$ Vgl. Holzmann, R. (2019), S. 6; Rommerskirchen, J. (2019), S. 16; Pieper, A. (2017), S. 21-22; Oermann, N. O. (2015), S. 11; Hentze, J. / Thies, B. (2012), S. 13; Noll, B. (2010), S. 25; Höffe, O. (2008a), S. 71; Rohls, J. (1999), S. 1.

$35 \mathrm{Vgl}$. Aristoteles (2015).
} 


\subsubsection{Ethisch und Moralisch}

Während in Bezug auf die genannten Substantive Ethik und Moral sowie Ethos ein relativ weitgehender begrifflicher Konsens in der wissenschaftlichen Literatur vorherrscht, gilt dies speziell im Zusammenhang mit den Adjektiven (un-) ethisch bzw. (un-)moralisch und den korrespondierenden Substantivierungen des Ethischen bzw. Moralischen nicht mehr. ${ }^{36}$

Eine Durchsicht sowohl deutsch- als auch englischsprachiger Fachbeiträge zeigt, dass die Begriffsverwendung in mehrere Klassen gegliedert werden kann. So verwenden viele Autoren die beiden Begrifflichkeiten relativ synonym ${ }^{37}$ oder benutzen lediglich einen der beiden Begriffe, meist jedoch, ohne die potentiellen begrifflichen Differenzen zu problematisieren. ${ }^{38}$ Dagegen vertritt beispielsweise HÜBNER die Auffassung, dass die beiden Adjektive moralisch und ethisch streng mit der aufgezeigten Begriffsauffassung der Substantive Moral und Ethik zu verknüpfen sind. Der Begriff „moralisch“ impliziert dabei eine (oft wertende ${ }^{39}$ ) Übereinstimmung mit geltenden Moralvorstellungen, der Begriff „ethisch“ konträr hierzu eine wertfreie Zuweisung eines Untersuchungsobjektes zum Gegenstandsbereich der Ethik. Daher wäre beispielsweise die Bestimmung guter ${ }^{40}$ Charaktereigenschaften ein ethisches Problem, die Wirkung der Gravitation allerdings nicht. ${ }^{41}$ In diesem Zusammenhang betont HUBNER, dass „unethisch“ keine gute Wortverwendung darstelle, da analog keine ,verständliche Anwendung [...] für die Wörter , unphysikalisch` oder , unbiologisch ““42

\footnotetext{
${ }^{36}$ Dies gilt insbesondere für den englischsprachigen Bereich, in dem der Terminus ,ethical behaviour" geläufig ist und häufig im Sinne von „moral behaviour“ verwendet wird. Vgl. Hübner, D. (2018), S. 20.

${ }^{37}$ Vgl. hierzu auch explizit Birnbacher, D. (2013), S. 1; Birnbacher, D. (2011b), S. 9.

38 Vgl. z. B. Quante, M. (2017), S. 12-13; Gellerman, S. W. (1986), S. 85-86; Hoerster, N. (1982), S. 9. Vgl. hierzu auch die kritische Rezeption bei Frankena, W. K. (1973), S. 5-6.

${ }^{39}$ Vgl. Hübner, D. (2018), S. 14.

${ }^{40}$ Zum Begriff des „Guten“ vgl. Hofmann-Riedinger, M. (2011), S. 387-389.

${ }^{41}$ Vgl. Hübner, D. (2018), S. 18. Vgl. ähnlich auch Pieper, A. (2017), S. 23.

${ }^{42}$ Hübner, D. (2018), S. 18.
} 
existiere. Vielmehr sei in diesem Kontext die Verwendung von „nichtethisch“43 vorzuziehen. ${ }^{44}$

HABERMAS und andere Ethiker in der Tradition KANTS verwenden dagegen teilweise „ethisch“ im Sinne von Werthaltungen, die sich auf das „Sollen“ gegenüber sich selbst zur Erzielung eines guten Lebens beziehen, während „moralisch“ sich auf das richtige Handeln bzw. Verantwortung gegenüber anderen bezieht. ${ }^{45}$ Diese der üblichen Begriffsverwendung zuwiderlaufende Definition des Ethischen als Individualmoral kann jedoch möglicherweise dadurch erklärt werden, dass „ethisches Handeln“ in diesem Kontext auf das Ethos rekurriert und nicht auf die Ethik als wissenschaftliche Disziplin. ${ }^{46}$

Schließlich kann auch ein Begriffsverständnis, welches die Ethik - durchaus in der Tradition KANTS - als vernunftbegründetes Nachdenken über Moral auffasst, zugrunde gelegt werden. Eine solche Position wird prinzipiell von KÜPPER vertreten. Er weist zwar darauf hin, dass die Begriffe „moralisch“ und „ethisch“ vielfach synonym gebraucht werden, entschließt sich dann aber dazu, den Begriff „ethisch“ für aus normativer Perspektive Zulässiges zu verwenden, weil dieser Begriff ,,in seiner Bedeutung und Anspruch weniger belastet ist ${ }^{\star 47}$ als der Begriff des Moralischen. ${ }^{48}$ Zudem betone der Begriff des Ethischen die Wissenschaftlichkeit in der Reflexion normativer Aussagen. ${ }^{49}$

An dieses Begriffsverständnis kann nun angeknüpft werden, wobei im Rahmen dieser Arbeit ein im Vergleich zu KÜPPER verfeinertes Argument zur Verwendung des Begriffes ,ethisch“ zum Einsatz kommt, insbesondere auch, um eine Konfusion mit der rein klassifikatorischen Begriffsverwendung, wie z. B. von HÜBNER hervorgehoben, zu vermeiden. Ausgangspunkt ist hierbei ebenfalls die Überlegung, dass wissenschaftliche Aussagen bestimmten Anforderungen unterliegen, welche sie von bloßen Meinungen abgrenzen. Wie im vorigen Kapitel dargelegt

\footnotetext{
${ }^{43}$ Der korrespondierende Begriff im Kontext der Moral wäre dabei amoralisch statt unmoralisch. Vgl. Birnbacher, D. (2013), S. 8-9. Vgl. auch Knoepffler, N. (2006), S. 10, der jedoch den Begriff ,außermoralisch“ vorschlägt, sowie Vieth, A. (2006), S. 11, welcher den Begriff ,nichtmoralisch“ im Sinne von HÜBNERS Auffassung des Begriffs „,nichtethisch“ gebraucht.

${ }^{44}$ Vgl. Hübner, D. (2018), S. 18.

${ }^{45}$ Vgl. Habermas, J. (2015), S. 104-105. Diese Unterscheidung findet sich auch bei anderen in der kantischen Tradition stehenden Ethikern wie RAWLS. Vgl. auch Celikates, R. / Gosepath, S. (2009), S. 12; Schweppenhäuser, G. (2006), S. 28.

46 Vgl. Hübner, D. (2018), S. 20-21.

${ }^{47}$ Küpper, H.-U. (2011), S. 18. Vgl. hierzu auch Ott, K. (2018), S. 7.

48 Vgl. ähnlich auch Birnbacher, D. (2013), S. 1.

${ }^{49}$ Vgl. Küpper, H.-U. (2011), S. 18.
} 
wurde, müssen wissenschaftliche Aussagen intersubjektiv nachvollziehbar und prüfbar sein, sie müssen innerhalb eines wissenschaftlichen Systems eingeordnet sowie mittels wissenschaftlicher Methoden gewonnen werden. ${ }^{50}$ Die konkrete Prüfbarkeit einer Aussage hängt jedoch entscheidend von ihrer Art ab. So gilt, wie diskutiert wurde, im Rahmen normativer Aussagen, dass deren Richtigkeit weder empirisch noch formal begründet werden kann, sondern immer im Austausch von Gründen zwischen den argumentierenden Individuen beruht. ${ }^{51}$ Folglich gelangt man zur Notwendigkeit der Begründung bzw. des Austauschs von Gründen. ${ }^{52}$ Gründe sind in der Praxis der zwischenmenschlichen Verständigung von großer Bedeutung. Sie sind in vielen Bereichen der Kommunikation, insbesondere bei der Rechtfertigung von Standpunkten sowie von Handlungen zentral. BRANDOM fasst dies pointiert zusammen ${ }^{53}$ : ,Asserting is giving reasons [...]."54 Dies impliziert ,making claims whose availability as reasons for others is essential to their assertional force. Assertions are essentially fit to be reasons. "55

Diese Überlegungen korrespondieren auch mit dem für die aufklärerische Philosophie zentralen Begriff ${ }^{56}$ des rationalen Handelns ${ }^{57}$, welcher wiederum durch die Existenz eines die Handlung erklärenden, guten Grundes definiert ist. ${ }^{58}$ Handlungsgründe weisen zwei wesentliche Funktionen auf: Sie müssen zum einen innerhalb der handelnden Person ein Motiv darstellen (motivationale Bedingung), andererseits aber auch intersubjektiv als Rechtfertigungsgrund dienen können

${ }^{50}$ Vgl. hierzu auch die Ausführungen bei Bardmann, M. (2019), S. 64-65; Kornmeier, M. (2007), S. 4-5.

51 Vgl. Küpper, H.-U. (2011), S. 41-42.

52 NIDA-RUMELIN spricht hier auch vom „Geben und Nehmen von Gründen“ (Nida-Rümelin, J. (2016b), S. 16), welches eine Übersetzung der gebräuchlichen englischen Formulierung des ,giving and taking reasons“ darstellt (vgl. z. B. Martin, A. M. (2014), S. 122; Buchanan, A. (2013), S. 137). Vgl. auch Brandom, R. B. (1994), S. 158, der dies als ,giving and asking for reasons" formuliert.

53 Ähnlich betont auch PAUER-STUDER: „Rechtfertigung unseres Tuns bedeutet immer, Gründe anzuführen, die auch Kraft für andere haben und die von anderen vernünftigerweise nicht zurückgewiesen werden können.“ (Pauer-Studer, H. (2020), S. 241).

${ }^{54}$ Brandom, R. B. (1994), S. 168.

55 Brandom, R. B. (1994), S. 168, in der Quelle ebenfalls herv.

${ }^{56}$ Der Begriff der Rationalität selbst gilt dabei als ,ein Zentralbegriff der Philosophie und Erkenntnistheorie.“ (Burkatzki, E. / Löhr, A. (2015), S. 13).

${ }^{57}$ In diesem Kontext ist die Abgrenzung von Irrationalität und Arationalität bedeutsam. Während Irrationalität den Verstoß gegen eine Rationalitätsnorm bezeichnet, meint der Begriff der Arationalität eine Nichtzugehörigkeit zum Bereich der Rationalität. Vgl. Davidson, D. (1999), S. 209; Gosepath, S. (1999), S. 8; Gosepath, S. (1992), S. 21.

58 Vgl. Nida-Rümelin, J. (2012), S. 11. 
(normative Bedingung). ${ }^{59}$ Hieran anknüpfend können in der Literatur zwei Hauptpositionen hinsichtlich des Begriffes „Grund“ unterschieden werden. Während die internalistische Position annimmt, dass die motivationale Funktion mit ihrer Rechtfertigung übereinstimmt und postuliert, dass sämtliches Handeln mit subjektiven Wünschen beginnt, vertritt die externalistische Position die Auffassung, dass sehr wohl subjekt-unabhängige Gründe außerhalb der motivlichen Situation der handelnden Person existieren, welche den normativen Status einer Handlung definieren (z. B. in Form eines kategorischen Imperativs). Eine solche Position findet sich im Kontext der Ethik beispielsweise bei der deontologischen Konzeption KANTS. ${ }^{60}$

In Anschluss an NIDA-RÜMELIN soll im Rahmen dieser Arbeit ein „Grund“ als eine Basisbegrifflichkeit (ähnlich wie „das Gute“) aufgefasst werden ${ }^{61}$, die nicht weiter sinnvoll elementar zu definieren ist, allerdings, wie oben ausgeführt wurde, durch ihre motivationalen und rechtfertigenden Eigenschaften charakterisiert werden kann. NIDA-RÜMELINS Rationalitätskonzept beruht dabei auf einer externalistischen Theorie der Gründe ${ }^{62}$, also der Annahme, dass Gründe nicht rein subjektive Motive darstellen, sondern objektiven Charakter annehmen. ${ }^{63}$ So besitzen hinsichtlich der Angemessenheit und Überzeugungskraft eines Grundes in einer spezifischen Situation Individuen zwar eine bestimmte Meinung, die allerdings, analog zur Meinung über die Richtigkeit einer Tatsache, den objektiven Kern eines Grundes nicht berührt. ${ }^{64}$ Gute Gründe sind hierbei Gründe, welche von einer Vielzahl an Individuen in einer bestimmten Situation als überzeugend erachtet bzw. faktisch geteilt werden ${ }^{65}$. Diese sind folglich Gründe, welche eine hohe Überzeugungskraft aufweisen, bzw. ,reziprok-allgemein nicht

\footnotetext{
${ }^{59}$ Vgl. Gosepath, S. (2012), S. 147; Heuer, U. (2001), S. 11; Gosepath, S. (1999), S. 10-11.

${ }^{60}$ Vgl. Gosepath, S. (1999), S. 13-14.

${ }^{61}$ Vgl. Gosepath, S. (2012), S. 150-151. Vgl. auch Horster, D. (2009), S. 101-103.

${ }^{62}$ Vgl. Nida-Rümelin, J. (2001), S. 73.

${ }^{63}$ Vgl. Nida-Rümelin, J. (2008), S. 20.

${ }^{64}$ Vgl. Nida-Rümelin, J. (2001), S. 35 und S. 78.

${ }^{65}$ Vgl. Nida-Rümelin, J. (2016a), S. 95.
} 
zurück-weisbar[]“66 sind. Solche Gründe können sich die kommunizierenden Personen zu eigen machen ${ }^{67}$ und sie können neben der internalistisch motivationalen Wirkung auch der Rechtfertigung von Handlungen dienen. ${ }^{68}$

Basierend auf diesen Vorüberlegungen sollen nun die Überlegungen zur terminologischen Präzision nach HUBBNER mit einem erweiterten Begriffsverständnis (so z. B. KUPPER) kombiniert werden. Der Terminus ,ethisch“ soll im Weiteren klassifikatorisch als dem Gebiet der ethischen Reflexion zugehörig gebraucht werden (z. B. im Sinne einer ,ethischen“ Problemstellung). Als Beurteilung, dass eine Handlung, eine Gesinnung bzw. eine Folge begründet zulässig bzw. wünschenswert sei, soll im Anschluss an diese Vorüberlegungen in dieser Arbeit aus terminologischen Präzisionsgründen jedoch eine adverbiale Begriffsverwendung wie ,ethisch fundiert“ oder ,ethisch begründet“" gebraucht werden, um eine Konfusion mit der rein klassifikatorischen Begriffsverwendung zu vermeiden. ${ }^{69}$ Der Begriff der ethischen Fundiertheit kann dabei vernunftbasiert aufgefasst werden, als auf eine normative Aussage referierend, welche mit guten Gründen (also insbesondere auch anerkannten Ethikkonzeptionen) gerechtfertigt werden kann. ${ }^{70}$ Es handelt sich folglich um ein systematisch begründetes und differenziertes Urteil. Hierbei spielt eine analytische Perspektive, welche das Argumentieren mit solchen Gründen ermöglicht, eine bedeutende Rolle, wie später im Rahmen der zu konzipierenden Unternehmensethik weiter ausgeführt wird. Dahingegen stellt das Moralische bzw. die Aussage, etwas sei „moralisch“, lediglich eine Übereinstimmung mit spezifischen Normenvorstellungen dar, welche empirisch in einer

\footnotetext{
${ }^{66}$ Forst, R. (2007), S. 41.

${ }^{67}$ NIDA-RUMELIN spricht hier von einer Fähigkeit, ,sich von Gründen affizieren zu lassen“ (Nida-Rümelin, J. (2016a), S. 257) bzw. einem „Zueigenmachen von Gründen“ (NidaRümelin, J. (2005b), S. 54) als Spezifikum der menschlichen Freiheit und Verantwortungsfähigkeit.

${ }^{68}$ Handlungen werden von NIDA-RUMELIN gerade durch die Existenz von Gründen gegenüber reinem Verhalten abgegrenzt (vgl. Nida-Rümelin, J. (2001), S. 75, S. 82 und S. 151). Die Auffassung von Handeln als zielorientierter intentionaler Tätigkeit, im Vergleich zum bloßen Verhalten, ist in der Literatur der Ethik und in der Soziologie weitverbreitet (vgl. Fenner, D. (2020), S. 38-39; Holzmann, R. (2019), S. 5; Nida-Rümelin, J. (2016a), S. 281).

${ }^{69}$ Einer differenzierenden Taxonomie folgt z. B. ebenso VÖNEKY, welcher betont, ,,dass [...] es auch , unethische ' Moral, d. h. unberechtigte moralische Urteile geben kann. ,Unethische Moral' sind Moralvorstellungen einer Gesellschaft, Gemeinschaft oder eines Individuums, die nicht durch ethische Kriterien gerechtfertigt werden können." (Vöneky, S. (2010), S. 28). ${ }^{70}$ Ausgenommen sei die Anwendung dieser Begriffstaxonomie in Fällen der direkten Zitation, in welcher die Originalterminologien im Weiteren beibehalten seien.
} 
bestimmten sozialen Einheit (wie einer Gruppe) existieren, ohne jedoch notwendigerweise universale Geltung aus guten Gründen, wie beispielsweise einen Rekurs auf allgemein anerkannte Basisnormen, beanspruchen zu können. Diese Überlegungen fasst nochmals die nachfolgende Abbildung 3.2 zusammen.

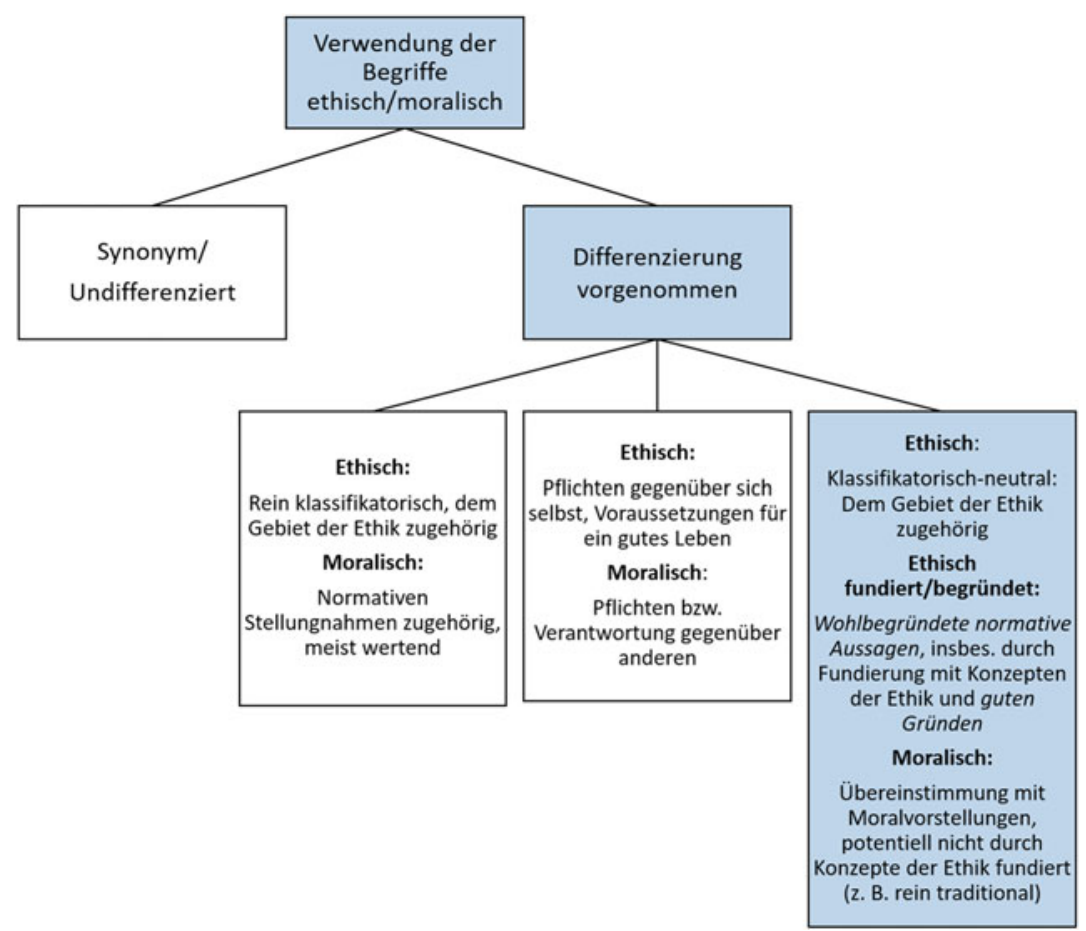

Abbildung 3.2 Taxonomie der Verwendungsmöglichkeiten der Begriffe „ethisch“ und „moralisch“ 


\subsection{Systematisierung der Ethik: Von der allgemeinen zur angewandten Ethik}

\subsubsection{Forschungsrichtungen der Ethik}

Versucht man die vielfältigen, möglichen Zugänge zur Ethik als wissenschaftlicher Disziplin zu systematisieren, so erscheint in einem ersten Schritt eine Differenzierung zwischen allgemeiner ${ }^{71}$ und angewandter Ethik fruchtbar. ${ }^{72}$ Während die allgemeine Ethik die konzeptionellen, aber auch empirischen Grundlagen der eigenen Disziplin erarbeitet sowie diese ggf. auch systematisch reflektiert, findet im Kontext der angewandten Ethik ein Transfer dieser Grundlagen auf eine fachspezifische Problemdomäne statt. ${ }^{73}$

Die allgemeine ethische Forschung wird häufig in drei Hauptrichtungen unterteilt. ${ }^{74}$ Die deskriptive Ethik befasst sich in diesem Zusammenhang mit konkret vorhandenen moralischen Normen, welche in einem gewissen Kulturkreis oder innerhalb einer Gemeinschaft existieren (,was ist“) ${ }^{75}$ Sie entspricht folglich einer empirischen Wissenschaft ${ }^{76}$, welche vorhandene Moralen untersucht, ohne

\footnotetext{
${ }^{71}$ Von einigen Autoren wird diese auch als philosophische Ethik bezeichnet. So z. B. bei Hübner, D. (2018), S. 21-22; Quante, M. (2017), S. 16; Fenner, D. (2010), S. 2-3. PIEPER bezeichnet diese auch als ,autonome Ethik“ (vgl. Pieper, A. (2017), S. 78) und NIDARUMELIN als ,theoretische Ethik“ (vgl. Nida-Rümelin, J. (2008), S. 37, vgl. auch Grübler, G. (2011), S. 308).

${ }^{72}$ Vgl. Fenner, D. (2020), S. 21-22; Ricken, F. (2013), S. 20; Thurnherr, U. (2010), S. 7-11; Celikates, R. / Gosepath, S. (2009), S. 12; Vieth, A. (2006), S. 7; Pieper, A. / Thurnherr, U. (1998), S. 9. Vgl. auch Knoepffler, N. (2006), S. 10, welcher diesen jedoch die Meta-Ethik direkt gegenüberstellt.

${ }^{73}$ In diesem Sinne betonen auch ZIMMERLI und AßLÁNDER, dass es Aufgabe einer angewandten Ethik sei, ,die formalen apriorisch entwickelten Überlegungen der Ethik auf konkrete Anwendungssituationen zu beziehen." (Zimmerli, W. C. I Aßländer, M. S. (2005), S. 303).

74 Vgl. Fenner, D. (2020), S. 22; Henning, T. (2019), S. 27-28; Härle, W. (2018b), S. 1012; Hübner, D. (2018), S. 21-23; Ott, K. (2018), S. 28; Veith, W. (2018), S. 5; Quante, M. (2017), S. 16; Rüther, M. (2015), S. 21; Bak, P. M. (2014), S. 7-12; Hentze, J. / Thies, B. (2012), S. 25; Düwell, M. / Hübenthal, C. / Werner, M. H. (2011), S. 2; Rehbock, T. (2011), S. 236-237; Rich, A. (2006), S. 11; Waibl, E. (2005), S. 35; Kreikebaum, H. (1996a), S. 11; Frankena, W. K. (1973), S. 4. Vgl. auch Göbel, E. (2020), S. 36-38, welche auch noch viertens die Methodenlehre als Teildisziplin der Ethik aufführt.

${ }^{75}$ Vgl. Hübner, D. (2018), S. 22.

${ }^{76}$ So wird diese von HENTZE und THIES auch ,empirische Ethik“ genannt (vgl. Hentze, J. / Thies, B. (2012), S. 25).
} 
jedoch die Untersuchungsergebnisse zu bewerten. Aus diesem Grund wird vielfach die Frage aufgeworfen, ob es sich bei einer solchen Ethik überhaupt noch um Philosophie oder nicht eher um eine Disziplin der empirischen Sozialforschung oder der Ethnologie handele. ${ }^{77}$ In der normativen Perspektive geht es dagegen um die Begründung von Moral. Die normative Ethik fokussiert demnach auf die Begründung und Legitimation von bestimmten Wertvorstellungen (,was sein soll“ und „,warum“). Sie benötigt daher einen anderen Zugang als die deskriptive Ethik. ${ }^{78}$ Während die deskriptive Ethik empirisch überprüf- und (im Sinne POPPERS) falsifizierbare ${ }^{79}$ Ergebnisse liefert, muss die normative Ethik auf Argumente zurückgreifen, welche nicht notwendigerweise intersubjektive Geltung beanspruchen können. ${ }^{80}$ Hierzu ist, wie bereits erörtert wurde, folglich der Austausch von Gründen zentral. Schließlich existiert mit der Metaethik auch eine philosophische Reflexion über den Status der Ethik und ihrer Begriffe. ${ }^{81}$ Während die Ethik dementsprechend die systematische Reflexion über Moral darstellt, repräsentiert die Metaethik eine Reflexion der Ethik selbst. ${ }^{82}$ Sie entspricht in diesem Sinne einer „Philosophie der Moralphilosophie“. So wird in diesem Kontext beispielsweise die ethische Bedeutung des Begriffes ,gut" diskutiert. ${ }^{83}$ Die Aufgabe der Metaethik kann auch in der klassischen, z. B. von ALBERT aufgegriffenen, Unterscheidung zwischen Objekt- und Metasprache repräsentiert werden. Die Metaethik verhält sich dabei zur Ethik wie die Metasprache zur Objektsprache. ${ }^{84}$ Sie steht damit in einem ähnlichen Verhältnis wie die Ethik als Metasprache zu den Normen, welche wiederum das Objekt der Ethik darstellen. ${ }^{85}$

\footnotetext{
77 Vgl. Fenner, D. (2020), S. 20; Göbel, E. (2020), S. 36; Hepfer, K. (2008), S. 23-24.

${ }^{78}$ Vgl. Hübner, D. (2018), S. 22.

${ }^{79}$ Vgl. hierzu Popper, K. R. (2005), S. 16-19.

${ }^{80} \mathrm{Zu}$ einer wissenschaftstheoretischen Reflexion vgl. Küpper, H.-U. (2011), S. 41-44.

${ }^{81}$ Wie die Literatur hervorhebt, handelt es sich bei der Metaethik im Vergleich zu den beiden anderen Forschungssträngen der Ethik (und insbesondere der normativen Ethik) um eine noch recht junge Disziplin. Vgl. hierzu Fenner, D. (2020), S. 20, welche betont, dass die Metaethik auch als „Wissenschaftstheorie der Ethik“ aufgefasst wird. Vgl. auch Lang, P. C. / Helferich, C. (2012), S. 485; Düwell, M. / Hübenthal, C. / Werner, M. H. (2011), S. 3.

${ }^{82}$ Vgl. Pauer-Studer, H. (2020), S. 205-206; Hübner, D. (2018), S. 37; Mizzoni, J. (2017), S. 184-188; Rüther, M. (2015), S. 14-16; Van Roojen, M. (2015), S. 1-3; Fisher, A. (2014), S. 1-4; Birnbacher, D. (2013), S. 58-59; Davis, P. (2008), S. 9-10; Hepfer, K. (2008), S. 15; Vieth, A. (2006), S. 13; Andersen, S. (2005), S. 7; Seiffert, H. (2001), S. 71-72; Frankena, W. K. (1973), S. 95-96. Vgl. auch die umfassende Einführung bei Kühler, M. (2016).

${ }^{83}$ Vgl. hierzu die weitere Diskussion in Hübner, D. (2018), S. 51-59.

${ }^{84}$ Vgl. Albert, H. (1979a), S. 473-476.

${ }^{85}$ Vgl. Düwell, M. / Hübenthal, C. / Werner, M. H. (2011), S. 2. Vgl. ähnlich auch NidaRümelin, J. (1995), S. 139.
} 
Im Rahmen dieser Arbeit werden insbesondere die deskriptive und normative Ethik von Relevanz sein, wohingegen metaethische Fragestellungen im Weiteren vernachlässigt werden.

Der allgemeinen Ethik kann wiederum die angewandte Ethik gegenübergestellt werden, welche eine wesentlich stärkere Praxisorientierung aufweist und sich durch Anwendung der allgemeinen Erkenntnisse auf eine spezifische Problemdomäne auszeichnet. Dabei sind hinsichtlich der Anwendungsbreite einer angewandten Ethik kaum Grenzen gesetzt: Diese reichen von medizinischen, technologischen, sozialethischen bis hin $\mathrm{zu}$ wirtschaftsethischen Fragestellungen. Die aufgezeigten klassifikatorischen Überlegungen fasst auch nochmals die nachfolgende Abbildung 3.3 zusammen.

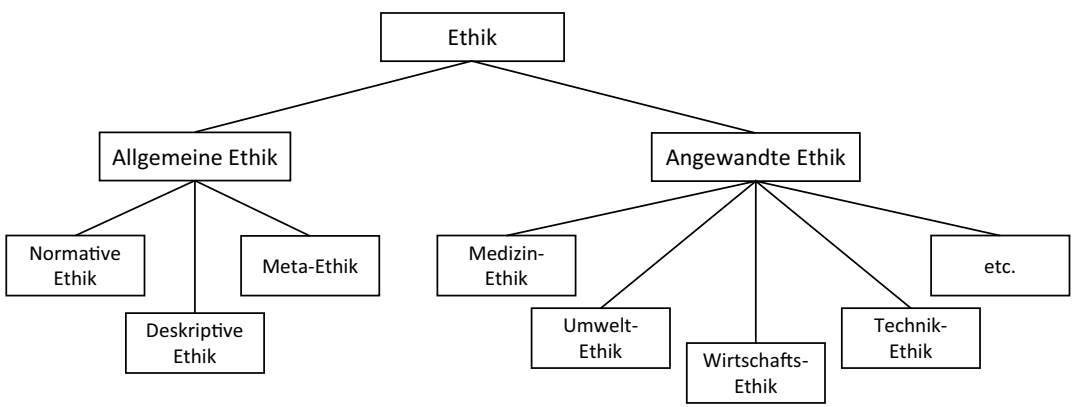

Abbildung 3.3 Überblick über die verschiedenen Formen der Ethik ${ }^{86}$

\subsubsection{Grundzüge der normativen Ethik als traditionelle Ausprägung der allgemeinen Ethik}

\subsubsection{1 Überblick über die Hauptströmungen}

Die älteste Forschungsrichtung der allgemeinen Ethik stellt die normative Ethik dar, auf welche im Weiteren näher eingegangen wird. Wie einleitend bereits erörtert wurde, befasst sich die normative Ethik mit normativen Aussagen, welche folglich auf ein "Sollen“ ausgerichtet sind. Pointiert kann man den Gegenstandsbereich dieser Richtung der Ethik nach KANT mit der Klärung der Frage:

\footnotetext{
${ }^{86}$ Vgl. ähnlich auch Fenner, D. (2020), S. 22; Pech, J. C. (2007), S. 34; Pieper, A. / Thurn-
} herr, U. (1998), S. 9. 
„Was soll ich thun?“87 zusammenfassen. Zur Beantwortung dieser Fragestellung hat die philosophische Forschung innerhalb ihrer langen Entstehungsgeschichte vielfältige, teils widerstreitende und unvereinbar erscheinende Ansätze bzw. Strömungen hervorgebracht. ${ }^{88}$ Dabei ist jedoch auch die Klassifikation dieser vielfältigen Konzeptionen selbst in der Literatur nicht unumstritten. Eine weitverbreitete, klassische Taxonomie differenziert hierbei die normativen Ethiken im Sinne einer Zweiteilung in deontologische, d. h. Pflichtenethiken ( $\delta$ źov [déon] = Pflicht), und teleologische Ethiken ( $\tau$ É $\mathrm{NOS}$ [télos] = Ziel) ${ }^{89}$

Die teleologischen Ethiken definieren das ethisch Richtige ${ }^{90}$ in dieser Klassifikation dabei als „Beitrag zur Realisierung oder Erhaltung eines Guten [.].“91 Im Rahmen teleologischer Ethiken sind daher primär die Folgen, d. h. die Erreichung eines anzustrebenden Zielzustandes ethischer Maßstab, während beispielsweise Motiven oder Handlungen alleine kein intrinsischer Wert zugemessen wird. Eine bekannte, wenn nicht sogar die bekannteste, teleologische Ethik stellt der Utilitarismus dar ${ }^{92}$, welcher vor allem von BENTHAM geprägt wurde. ${ }^{93}$ Grundgedanke ist bei BENTHAM, dass die Summe des Glücks als ethischer Maßstab gilt, nicht jedoch individuellen Motive oder Handlungen als solche. Zur ethischen Bewertung wird daher lediglich ,the greatest happiness of the greatest number“94 zugrunde gelegt. Dieser Ansatz wird in der Literatur auch als hedonistischer Utilitarismus bezeichnet. ${ }^{95}$ Neben der Fokussierung auf das Glück als zu maximierende Zielgröße wurden im Laufe der Zeit diverse Abänderungen

\footnotetext{
${ }^{87}$ Kant, I. (1968c), B 833, in der Quelle gesperrt herv.

${ }^{88}$ Vgl. nochmals Arnold, D. G. / Beauchamp, T. L. / Bowie, N. E. (2020), S. 33.

${ }^{89}$ Vgl. z. B. Pauer-Studer, H. (2020), S. 15; Düwell, M. / Hübenthal, C. / Werner, M. H. (2011), S. 16; Hepfer, K. (2008), S. 30; Kreikebaum, H. (2006), S. 16; Noll, B. (2002), S. 1516; Kreikebaum, H. (1996a), S. 12; French, W. A. / Granrose, J. (1995), S. 10-12; Steinmann, H. / Löhr, A. (1994a), S. 13; Frankena, W. K. (1973), S. 14.

${ }^{90}$ Dies ist vom „Guten“, insbesondere bei deontologischen Ethiken seit KANT, zu unterscheiden. Vgl. Hofmann-Riedinger, M. (2011), S. 387. Vgl. zu dieser Differenzierung auch Ross, W. D. (2002), S. 1-15 und S. 65-74. Bei älteren Ansätzen, z. B. der klassischen Antike, ist eine solche Unterscheidung jedoch nicht in den Theorien vorgesehen und eine nachträgliche Anwendung dieser Differenzierung somit kritisch zu sehen (vgl. Düwell, M. / Hübenthal, C. / Werner, M. H. (2011), S. 17).

${ }^{91}$ Düwell, M. / Hübenthal, C. / Werner, M. H. (2011), S. 16. Vgl. einführend auch Hübenthal, C. (2011).

92 Vgl. Hübner, D. (2018), S. 211. Vgl. ähnlich auch Pieper, A. (2017), S. 164.

93 Vgl. Hübner, D. (2018), S. 237.

94 Bentham, J. (2010), S. 393.

${ }^{95}$ Vgl. Smart, J. J. C. / Williams, B. (1998), S. 12.
} 
des Nützlichkeitskalküls ${ }^{96}$ entwickelt. So kann neben einer Fokussierung auf die Nützlichkeit einer konkreten Handlung (s. g. Akt-Utilitarismus) auch die Nützlichkeit im Falle, dass verallgemeinert so gehandelt würde (s. g. RegelUtilitarismus), zugrunde gelegt werden. ${ }^{97}$ Zudem kann anstatt des hedonistischen Strebens nach Glück auch allgemein eine Maximierung des Nutzens, welcher anhand von (prinzipiell beliebigen) Präferenzen modelliert wird, stattfinden. Gerade letztere Variante des Utilitarismus, der s. g. Präferenz-Utilitarismus, nimmt insbesondere in der Ökonomik mit der traditionellen Maximierung von Nutzenfunktionen ${ }^{98}$ eine zentrale Position ein ${ }^{99}$ - wenngleich von traditionellen Vertretern des Fachs häufig eine Verbindung zur Ethik bestritten wird. ${ }^{100}$

In Abgrenzung zu den teleologischen Ethiken werden zumeist die deontologischen Ethiken genannt. Kerncharakteristikum deontologischer Ethiken ist „die Auffassung, dass Handlungen aufgrund anderer Charakteristika als ihrer konkreten Folgen [.] richtig oder falsch sind." ${ }^{101}$ Der bedeutendste ${ }^{102}$ Vertreter der deontologischen Ethiken ist hierbei IMMANUEL KANT, welcher das richtige Handeln aus dem richtigen Motiv des Pflichtgefühls heraus und der Orientierung an universalisierbaren Maximen betonte. Das Pflichtgemäße muss damit in der Terminologie KANTS aus Pflichtgefühl getan werden und nicht etwa aus Affekt oder gar aus eigennützigen Überlegungen. Nur die richtige Handlung um ihrer selbst willen hat daher nach KANT einen ethischen Wert. ${ }^{103}$ Dabei spielt der gute Wille

\footnotetext{
${ }^{96}$ So stammt auch der Begriff des Utilitarismus von lateinischen ,utilitas“ bzw. englischen „utility“ ab. Vgl. Hübner, D. (2018), S. 212.

97 Vgl. Hübner, D. (2018), S. 215 und S. 220; Vieth, A. (2018), S. 84-86; Höffe, O. (2013), S. 28-40; Singer, P. (2011), S. 232; Horster, D. (2009), S. 41-46; Lyons, D. (2002), S. 119121; Smart, J. J. C. / Williams, B. (1998), S. 9-12; Slote, M. (1995), S. 58-59; Frankena, W. K. (1973), S. 35-43.

${ }^{98}$ So z. B. in der mikroökonomischen Produktions- oder Konsumptionstheorie (vgl. z. B. Breyer, F. (2020), S. 144-148; Siebe, T. (2016), S. 118; Herdzina, K. / Seiter, S. (2015), S. 74; Varian, H. R. (2014), S. 74-78; Hall, R. E. / Lieberman, M. (2013), S. 152; Piekenbrock, D. / Hennig, A. (2013), S. 98) sowie in der klassischen normativen Entscheidungstheorie (vgl. z. B. Bamberg, G. / Coenenberg, A. G. / Krapp, M. (2019), S. 32-33; Göbel, E. (2016), S. 110-118; Laux, H. / Gillenkirch, R. M. / Schenk-Mathes, H. Y. (2014), S. 74-76; Obermaier, R. / Saliger, E. (2013), S. 26-31; Wessler, M. (2012), S. 1).

${ }^{99}$ Vgl. Höffe, O. (2013), S. 9; Holstein, L. (2003), S. 175-176; Rothschild, K. W. (1992), S. 1.

${ }^{100}$ Vgl. hierzu nochmals die Ausführungen zur historischen Reflexion normativer Aussagen in der Betriebswirtschaftslehre im zweiten Kapitel dieser Arbeit.

${ }^{101}$ Düwell, M. / Hübenthal, C. / Werner, M. H. (2011), S. 16.

102 Vgl. Hentze, J. / Thies, B. (2012), S. 37.

${ }^{103}$ Vgl. Kant, I. (1968b), S. 397-398.
} 
nach KANT die zentrale Rolle bei der Bewertung von Handlungen ${ }^{104}$, was ihm den Vorwurf eingebracht hat, lediglich Gesinnungsethiker ${ }^{105}$ zu sein. Eine solche Auffassung greift jedoch zu kurz, denn der ,gute Wille“ ist keineswegs beliebig oder rein subjektiv zu begreifen, sondern wird im Rahmen von KANTS deontologischer Konzeption durch den kategorischen Imperativ präzisiert. ${ }^{106}$ D. h. ein guter Wille ist nach KANT ausschließlich der Wille, der aus Pflichtgefühl ,nur nach derjenigen Maxime [handelt], durch die du zugleich wollen kannst, daß sie ein allgemeines Gesetz werde." ${ }^{107}$

Die aufgezeigten zwei zentralen Theorietraditionen der normativen Ethik finden sich in der Literatur mit vielfältigen ähnlichen Systematisierungen wieder. So wird beispielsweise die teleologische Ethik oftmals auch Folgen- oder Verantwortungsethik genannt. Die Deontologie wird dagegen häufig als Pflichtenethik bezeichnet. Obwohl bei genauerer Betrachtung die Begrifflichkeiten nicht immer vollständig synonym aufzufassen sind, ergeben sich dennoch große Ähnlichkeiten mit den beiden großen Theorietraditionen. ${ }^{108}$ Aus den genannten Abgrenzungskriterien dieser zentralen normativen Theorierichtungen wird allerdings nicht unmittelbar klar, ob nicht noch weitere normative Ethiken existieren können, die nach den aufgezeigten Definitionen ,weder deontologisch noch teleologisch sind." 109 Aus diesem Grund wurde die aufgezeigte Begriffsabgrenzung von FRANKENA ${ }^{110}$ und RAWLS ${ }^{111}$ weiter konkretisiert und zu einer klaren Dichotomie verengt. Die Autoren definieren hierbei diejenigen Ansätze als teleologische Ethiken, ,die das moralisch Richtige als Funktion des vormoralisch Guten bestimmen" ${ }^{112}$, wobei das vormoralisch Gute dadurch definiert wird, dass

${ }^{104}$ So schreibt etwa KANT in seiner Grundlegung zur Metaphysik der Sitten: „Es ist überall nichts in der Welt, ja überhaupt auch außer derselben zu denken möglich, was ohne Einschränkung für gut könnte gehalten werden, als allein ein guter Wille." (Kant, I. (1968b), S. 393, in der Quelle fett herv).

105 Der Begriff „Gesinnungsethik“ sollte zudem, wie z. B. von GÖBEL oder HÜBNER praktiziert, besser in Verbindung mit Tugendethiken genutzt werden, da hier der Fokus auf die motivliche Komponente der Handlung gelegt wird (vgl. Göbel, E. (2020), S. 30 und S. 40-41; Hübner, D. (2018), S. 90).

106 Vgl. Göbel, E. (2020), S. 40-44; Blöser, C. (2014), S. 203; Höffe, O. (2012), S. 162; Fetzer, J. (2004), S. 77-78; Kaulbach, F. (1978), S. 309.

${ }^{107}$ Kant, I. (1968b), S. 421, in der Quelle gesperrt herv. Vgl. auch Kant, I. (1968e), S. 30.

108 Vgl. Hentze, J. / Thies, B. (2012), S. 32.

${ }^{109}$ Düwell, M. / Hübenthal, C. / Werner, M. H. (2011), S. 16.

${ }^{110}$ Vgl. Frankena, W. K. (1973), S. 14-17.

111 Vgl. Rawls, J. (1999), S. 21-22.

112 Düwell, M. / Hübenthal, C. / Werner, M. H. (2011), S. 16. 
es zur Begründung selbst keine ethischen Kategorien benötigt. So wäre z. B. Glück eine gegebene Zielgröße, welche exogen bestimmt ist. Deontologische Theorien werden in Abgrenzung hiervon durch die Negation der teleologischen Ethik bestimmt, so dass in dieser Taxonomie kein Raum für eine möglicherweise dritte Kategorie normativer Ethiken verbleibt. ${ }^{113}$

Es lassen sich jedoch überzeugende Gründe anführen, welche eine Zweiteilung normativer Ethiken als nicht hinreichend bewerten. Diese zeigen, warum eine dritte Ethiktradition von den bisherigen Hauptrichtungen abgegrenzt werden sollte, weil diese nicht sinnvollerweise unter einen der beiden anderen Theoriestränge subsumiert werden kann. So wird von einigen Autoren, wie beispielsweise HÜBNER $^{114}$ oder HENTZE und THIES ${ }^{115}$ eine Dreiteilung normativer Ethiken begründet. Diese Autoren unterscheiden neben den deontologischen und teleologischen Ethiken die Tugendethik in der Tradition des ARISTOTELES ${ }^{116}$ als dritte normative Grundkonzeption. ${ }^{117}$ Die Tugendethik stellt aus dieser Perspektive eine eigenständige normative Theorietradition dar, welche sich weder der Deontologie noch der Teleologie zurechnen lässt. Fokussiert man auf die Begründung der Ethik durch ARISTOTELES, so erscheint seine Tugendethik zwar auf den ersten Blick teleologisch, da er als oberstes Ziel die Glückseligkeit ( $\varepsilon \cup 3 \delta \alpha \iota \mu$ oví $\alpha$ [eudaimonía]) postuliert. ${ }^{118}$ Dieses Ziel ist jedoch nicht Grundlage der ethischen Bewertung von Handlungsfolgen (Zuständen), wie z. B. im Utilitarismus,

${ }^{113}$ Vgl. Düwell, M. / Hübenthal, C. / Werner, M. H. (2011), S. 17.

114 Vgl. Hübner, D. (2018), S. 88.

115 Vgl. Hentze, J. / Thies, B. (2012), S. 31.

116 Tugendethiken existierten bereits vor ARISTOTELES, beispielsweise bei seinem akademischen Lehrer Platon. Vgl. Hübner, D. (2018), S. 101-111. Dennoch kann, wie die häufige Referenz zeigt, die Tugendethik des ARISTOTELES als eine der bedeutendsten Konzeptionen und als grundlegend in der Philosophiegeschichte erachtet werden.

117 Vgl. zu einer solchen Auffassung auch Göbel, E. (2020), S. 40-41; Härle, W. (2018b), S. 76-85; Quante, M. (2017), S. 126-130; Birnbacher, D. (2013), S. 302-306; Fisher, C. I Lovell, A. (2013), S. 104. Vgl. auch Rommerskirchen, J. (2019), S. 40, der unter teleologischen Ethiken jedoch nur die aristotelische Ethik subsumiert. Vgl. zudem Höffe, O. (2018), S. 53-54; Suchanek, A. (2007), S. 15-20, welche sogar zwischen vier Grundmodellen unterscheiden. Vgl. ähnlich auch Nida-Rümelin, J. (1995), S. 85.

${ }^{118}$ Vgl. Hübner, D. (2018), S. 111. So ordnet auch ROMMERSKIRCHEN die Ethik des ARISTOTELES den teleologischen Ethiken zu, definiert utilitaristische Theorien jedoch als eigenständige Forschungstradition (vgl. Rommerskirchen, J. (2019), S. 40). 
sondern in dieser Perspektive liegt die Glückseligkeit bereits im Vollzug der richtigen Handlung an sich. ${ }^{119}$ Andererseits ist die aristotelische Ethik auch nicht der Deontologie zuzurechnen ${ }^{120}$, wenngleich diese durchaus deontologische Elemente enthält. ${ }^{121}$ Vielmehr steht im Mittelpunkt das Streben ${ }^{122}$ des Menschen nach charakterlicher Vollkommenheit bzw. der Aneignung der richtigen Motive zum Ausbau tugendhaften Handelns. ${ }^{123}$ Dabei ist in der ,reinen“ Tugendethik die Tugend ${ }^{124}$ in Abgrenzung zur Deontologie oder Teleologie keinesfalls nur als Mittel zum Handeln aus Pflicht oder zur Zielerreichung im Sinne des Utilitarismus anzusehen. Vielmehr weist sich die Tugendethik gerade dadurch aus, dass sie der Ausbildung der charakterlichen Vortrefflichkeit bzw. der Tugenden einen Wert an sich zuschreibt bzw. diese als primäre ethische Kategorie definiert. ${ }^{125}$ Die vorausgegangenen Überlegungen fasst nochmals die nachfolgende Abbildung 3.4 zusammen.

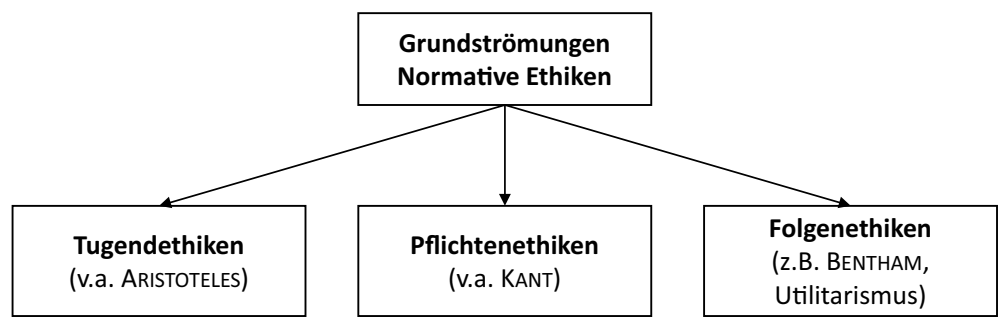

Abbildung 3.4 Die drei zentralen Strömungen der normativen Ethik

Neben diesen drei normativen Hauptrichtungen existieren noch weitere normative Strömungen innerhalb der Ethik, wie beispielsweise die Diskursethik als

${ }^{119}$ Vgl. Hübner, D. (2018), S. 114. ARISTOTELES spricht hier vom tugendhaften Tätigsein „,der Seele im Sinn der Gutheit [...], [d]enn das gute Handeln selbst ist Ziel.“ (Aristoteles (2015), 1102a und 1140b).

${ }^{120}$ Vgl. Hübner, D. (2018), S. 115.

121 Vgl. Rapp, C. (2011b), S. 79.

122 Die Tugendethik wird deshalb auch als Strebensethik bezeichnet (vgl. Hentze, J. / Thies, B. (2012), S. 33).

${ }^{123}$ Vgl. Hübner, D. (2018), S. 116; Hentze, J. / Thies, B. (2012), S. 33-34.

${ }^{124}$ Die Tugend kann dabei im Kern als Merkmal eines anzustrebenden Charakters aufgefasst werden. Vgl. Halbig, C. (2013), S. 34. Hierdurch ergibt sich ersichtlicherweise eine Verbindung zu den Handlungsmotiven einer Person.

125 Vgl. Hübner, D. (2018), S. 146-147. 
prozedurale Ethik oder auch der Ethische Egoismus, die Goldene Regel der Reziprozität sowie die stärker deskriptiv ausgerichtete Common Morality Theory ${ }^{126}$, welche postuliert, dass im Kern alle Menschen ähnliche Grundwerte teilen. ${ }^{127}$ Gerade letzterer Ansatz scheint dabei von besonderer Relevanz, welcher im Rahmen dieser Arbeit mit der Weltethos-Forschung ${ }^{128}$ eine Lösung zur Kritik normativer Ansätze offeriert, indem das Postulat einer ethischen Letztbegründung verworfen und mit einem pragmatisch-deskriptiven Ansatz substituiert wird. Die aufgezeigten Überlegungen werden im folgenden Unterkapitel nun in Grundzügen dargestellt, bevor diese wiederum im vierten Kapitel nochmals detaillierter zur Entwicklung einer eigenständigen Konzeption der Unternehmensethik wiederaufgegriffen werden.

\subsubsection{Bezug zum Handlungsprozess und die Grenzen der normativen Ethik}

Die zuvor aufgezeigte Dreiteilung der Ethiken ist nicht nur aus genuin konzeptionell-ethischer Perspektive fruchtbar. Vielmehr lassen sich die vorausgegangenen Überlegungen auch mit dem für Fragen der Verantwortungszuweisung bedeutsamen wie schillernden Begriff der Handlung verknüpfen. Im Kontext der sozialwissenschaftlichen wie philosophischen Forschung wird dabei vielfach der Begriff der Handlung vom reinen Verhalten abgegrenzt. In diesem Sinne bezeichnet das Handeln ein bewusst gesteuertes, intentionales, d. h. auf ein bestimmtes Ziel gerichtetes Tun, während reines Verhalten auch nicht-intentional sein kann und z. B. allgemeine Körperfunktionen widerspiegelt. ${ }^{129}$ Durch den Zusammenhang der Intentionalität ist somit eine Verantwortungszuschreibung an das

${ }^{126}$ Vgl. Arnold, D. G. / Beauchamp, T. L. / Bowie, N. E. (2020), S. 29-32; Beauchamp, T. L. / Childress, J. F. (2013), S. 2-5 und S. 410-411.

${ }^{127}$ Vgl. so z. B. die Systematisierungen in Arnold, D. G. / Beauchamp, T. L. / Bowie, N. E. (2020); Pauer-Studer, H. (2020); Crane, A. et al. (2019); Holzmann, R. (2019); Hübner, D. (2018); Boonin, D. / Oddie, G. (2010); Dutt, A. K. / Wilber, C. K. (2010); Celikates, R. / Gosepath, S. (2009); Düsing, K. (2005).

${ }^{128} \mathrm{Vgl}$. hierzu detailliert Abschnitt 4.5.2.

${ }^{129}$ Vgl. Fenner, D. (2020), S. 38-39; Brühl, R. (2017), S. 49; Steinmann, H. / Löhr, A. (2015), S. 277-278; Sombetzki, J. (2014), S. 47; Lehner, F. (2011), S. 131; Andersen, S. (2005), S. 11; Seiffert, H. (2001), S. 15-16; Günther, A. (1998), S. 221; Hubig, C. (1995), S. 42; Müller-Koch, U. (1991), S. 55-56. Vgl. auch die berühmte Definition WEBERS zum sozialen Handeln: ,,S Soziales “ Handeln aber soll ein solches Handeln heißen, welches seinem von dem oder den Handelnden gemeinten Sinn nach auf das Verhalten anderer bezogen wird und daran in seinem Ablauf orientiert ist.“ (Weber, M. (1976), S. 1, in der Quelle gesperrt herv.). 
handelnde Subjekt ${ }^{130}$ möglich ${ }^{131}$, da dieses sich potentiell auch hätte anders entscheiden können, sofern in der gegebenen Situation das Subjekt über ausreichend Dispositions- und Handlungsfreiheit verfügte. So betont auch ESSER: „Handeln ${ }^{132}$ in einer Situation ist [.] immer eine Entscheidung zwischen Alternativen. “133

In einem vereinfachten, in ähnlicher Darstellung auch im Bereich der Motivationsforschung weitverbreiteten Schema, ${ }^{134}$ kann dabei der Handlungsprozess in drei Phasen unterteilt werden. In einem ersten Schritt steht hierbei das Subjekt mit seinen charakterlichen Dispositionen bzw. Motiven im Fokus. ${ }^{135}$ In einem zweiten Schritt erfolgt dann eine Handlung, welche drittens zu bestimmten Folgen führt. Diese drei zentralen Ablaufschritte können nun wiederum den drei zentralen ethischen Grundströmungen zugeordnet werden. So befassen sich die Tugendethiken, z. B. klassisch im Falle von ARISTOTELES, im Kern mit den Dispositionen bzw. Motiven des Handelnden, während die Deontologien in der Tradition KANTS auf die Pflicht zu gewissen Handlungen fokussieren. Schließlich betonen Teleologien, wie z. B. der gerade in der Ökonomik weitverbreitete Utilitarismus ${ }^{136}$, die Bedeutung, eine Bewertung von Handlungen nur an den Konsequenzen, nicht jedoch an den Motiven oder der Handlung selbst, festzumachen. Diese Überlegungen fasst nochmals die nachfolgende Abbildung 3.5 zusammen. ${ }^{137}$

$\mathrm{Zu}$ klären wäre allerdings, in welchem Verhältnis die aufgezeigten Ethiken stehen. Im Falle einer Kongruenz der Beurteilungen scheint dies unproblematisch. So wären Handlungen, welche von allen Konzeptionen als richtig eingestuft werden, grundsätzlich auszuführen, Handlungen, welche von allen Konzeptionen

${ }^{130}$ Zur kritischen Würdigung des Subjektbegriffs und insbesondere auch zur Frage, wer als Subjekt in diesem Sinne gelten kann, vgl. die Ausführungen in Abschnitt 4.2.

131 Vgl. Fenner, D. (2020), S. 39.

${ }^{132} \mathrm{Zu}$ beachten ist, dass aus ethischer Perspektive auch eine Unterlassung als relevante Handlung, z. B. aufgrund positiver Handlungspflichten, gelten kann. Vgl. hierzu Fenner, D. (2020), S. 55-57; Härle, W. (2018b), S. 64.

${ }^{133}$ Esser, H. (1999), S. 5, in der Quelle ebenfalls herv.

${ }^{134}$ Vgl. z. B. Heckhausen, J. / Heckhausen, H. (2018), S. 6; Lingnau, V. / Willenbacher, P. (2013), S. 9. Vgl. aus soziologischer Perspektive auch Esser, H. (1999), S. 6 und im Kontext der Ethik auch Andersen, S. (2005), S. 12.

${ }^{135} \mathrm{Vgl}$. zum schillernden Begriff des Subjekts die detaillierten Ausführungen in Abschnitt 4.2.

${ }^{136}$ Konsequentialistische Überlegungen finden sich dabei auch in der Controllingforschung, so z. B. in der Controllingkonzeption von WEBER und SCHÄFFER (vgl. Lingnau, V. / Schäffer, U. (2009), S. 284).

${ }^{137}$ Vgl. zu ähnlichen Überlegungen auch Noll, B. (2013), S. 60. 


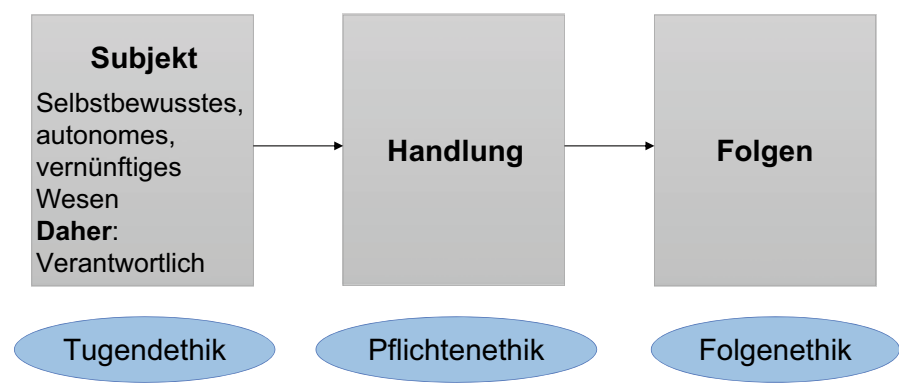

Abbildung 3.5 Der Zusammenhang der drei zentralen normativen Strömungen zum Handlungsprozess

kritisiert würden, wären zu unterlassen. Praktisch jedoch können aus den 3 Perspektiven insgesamt $2^{3}=8$ verschiedene Beurteilungskombinationen entstehen. In diesem Sinne kann z. B. eine Person aus ,guten“ Intentionen, wie dem Schutz von Arbeitsplätzen, in der Öffentlichkeit lügen und damit das Unternehmen retten. ${ }^{138}$ Aus tugendethischer Perspektive wie auch aus konsequentialistischer Sicht mag ein solches Handeln nun vertretbar sein, aus Sicht der kantischen Pflichtenethik hingegen nicht, da hier die Handlung des unwahrhaftigen Aussagens grundsätzlich und an sich einen Verstoß gegen ethische Handlungspflichten darstellt. Bereits in diesem Beispiel zeigt sich also, dass ein Handlungsprozess von verschiedenen Ethiken vollständig unterschiedlich bewertet werden kann.

Insgesamt lässt sich daher feststellen, dass die normative Ethikforschung zweifellos bedeutsame Beiträge zur Begründung normativer Standpunkte geliefert hat, jedoch vielfach das Problem für den Entscheider birgt, keine hinreichend sichere und allgemein gültige Begründung für die Zulässigkeit einer Handlung in der Praxis erzielen zu können. Hierdurch läuft eine rein normativ fundierte Legitimierung von Handlungen stets Gefahr, für praktische Entscheidungsprozesse „leer“ zu sein, d. h. letztlich nur verschiedene potentiell widerstreitende Argumente aufzuzeigen $^{139}$, aber kein fundiertes Urteil über die Zulässigkeit von Handlungen zu ermöglichen. ${ }^{140}$ In diesem Sinne betonte bereits COHN in den 1920er Jahren,

${ }^{138}$ Vgl. zu einem ähnlichen Beispiel Göbel, E. (2020), S. 39.

${ }^{139}$ Zudem sind die normativen Standpunkte selbst nicht letztbegründbar, wie das bereits diskutierte Münchhausen-Trilemma zeigt. Vgl. hierzu die Ausführungen in Abschnitt 2.5.1.

${ }^{140}$ Eine Möglichkeit wäre hier aus konzeptioneller Sicht die „Gesamtschau“ aller normativen Standpunkte, um aus diesen ein Urteil zu fällen (vgl. hierzu die Überlegungen der Triple Font Theory in Arjoon, S. (2007)). Diesen Ansatz verfolgen auch einige angelsächsische 
dass die Probleme normativer Ethik „trotz der ungeheuren Geistesarbeit, welche die größten Denker der Menschheit auf dieselben angewendet haben, ihrer Lösung nicht wesentlich näher gerückt sind. Während in den meisten anderen Wissenschaften ohne Zweifel im Laufe der Zeit eine [...] außerordentlich große Entwicklung nachgewiesen werden kann, [...] erörtert die Ethik noch ganz dieselben Fragen, die schon Sokrates [...] mit seinen Zuhörern besprach." ${ }^{\text {141 }}$ Zudem gäbe es „,im buchstäblichen Sinne kaum zwei Philosophen, die in Bezug auf die Prinzipien und die weitere Ausarbeitung der Ethik übereinstimmen, und ein jeder hat, bevor er sein eigenes positives System darstellt, erst alle andern, früheren und gleichzeitigen Moralbegründungen widerlegt." ${ }^{\text {142 }}$

Schließlich laufen rein normative Argumentationsstrategien, wie bspw. die wirtschaftsethische Schule von HOMANN zurecht betont ${ }^{143}$, vielfach Gefahr, in der Praxis als „Moralisierung“ wahrgenommen zu werden und können damit gerade die klassische Intention, in einem praktisch wirksamen Diskurs, die Lebensumstände der Menschen zu verbessern, nicht erfüllen. Ein Ansatz, welcher diese Schwierigkeiten zu umgehen vermag, könnte daher nicht mit einer rein normativen Begründung beginnen, sondern müsste von einer verstärkt deskriptiven Perspektive ausgehen, welche folglich zur praktischen Wirksamkeit die realen Moralvorstellungen mitberücksichtigt, diese aber auch durchaus kritisch in einem zweiten Schritt prüfen kann. Dieser Gedanke wird nochmals in der Ausarbeitung der eigenen unternehmensethischen Konzeption aufgegriffen und dort - insbesondere auch gegen den häufig artikulierten ethischen Relativismusvorwurf einer deskriptiven Perspektive - kritisch detaillierter vorgestellt. ${ }^{144}$

In einem weiteren Schritt ist im Rahmen dieser Arbeit jedoch zuerst der Forschungsstand der wirtschafts- und unternehmensethischen Forschung zu erarbeiten. Nach einer Reflexion der Grenzen einer rein normativen Ethik ist daher in einem nächsten Schritt, die angewandte Ethik als domänenspezifische Forschung im Bereich der Ethik zu erläutern. Dies ist Gegenstand des nachfolgenden Abschnitts.

Frameworks zur Integration dieser verschiedenen normativen Ethiken (so z. B. Fisher, C. / Lovell, A. (2013), S. 103). Problematisch bleibt allerdings auch hier, dass im Falle widersprüchlicher Evaluationen letztlich ein normatives Metaurteil notwendig bleibt, welches die Argumente ggf. priorisiert bzw. darüber entscheidet, wie die unterschiedlichen Standpunkte $\mathrm{zu}$ aggregieren wären.

${ }^{141}$ Cohn, G. (1923), S. 71-72.

142 Cohn, G. (1923), S. 73.

${ }^{143}$ Vgl. hierzu detailliert Abschnitt 3.4.3.

${ }^{144}$ Vgl. hierzu insbesondere die Ausführungen in Abschnitt 4.4 und 4.5. 


\subsubsection{Angewandte Ethik als domänenspezifische Applikation}

Neben der aufgezeigten Möglichkeit im Rahmen der allgemeinen Ethik grundlegende Erkenntnisse über das ethische Sollen oder die faktische Wirklichkeit der Moral in einer sozialen Einheit zu erfahren, hat sich mit der zunehmenden Ausdifferenzierung der modernen Gesellschaften ${ }^{145}$ die Notwendigkeit ergeben, in spezifischen Problemdomänen spezialisierte Teildisziplinen der Ethik als angewandte Ethiken zu entwickeln. In diesem Sinne betont auch HöFFE die öffentliche Relevanz der angewandten Ethik: „Das heutige Interesse an der Ethik richtet sich vor allem auf die Fragen der Wirtschaft, der Umwelt und der Medizin samt Technik. Derart angewandte Ethiken erfreuen sich eines bleibenden öffentlichen [...] Interesses." ${ }^{146}$ Die Teildisziplin der angewandten Ethik besitzt dabei eine fachhistorisch lange Tradition, welche sich bis in die Antike zurückverfolgen lässt, jedoch durch die zwischenzeitlich starke Formalisierung der Ethik und den Fokus auf abstrakte Grundlagenforschung vernachlässigt wurde. ${ }^{147}$ Als eigenständige Disziplin wurde die angewandte Ethik nach weitverbreiteter Auffassung in den 1970er Jahren im angelsächsischen Raum explizit wieder aufgegriffen, in welchem Zeitraum auch der Begriff „,applied ethics“ zunehmende Verbreitung erfuhr. ${ }^{148}$ Der deutschsprachige Begriff der angewandten Ethik stellt in diesem Zusammenhang eine direkte Übersetzung des englischen Terminus dar. ${ }^{149}$ Den Initialfunken zur Wiederaufnahme einer explizit problemorientierten Ethikforschung lieferte dabei im angelsächsischen Raum die in dieser Zeit intensive Kontroverse um die Nutzung neuer biomedizinischer Möglichkeiten ${ }^{150}$, welche eine Diskussion um die Zulässigkeit dieser Maßnahmen initiierte. Hierbei stand insbesondere im Fokus, keine abstrakten, sondern konkretisierte und zugleich ethisch fundierte Antworten auf drängende Fragen, wie z. B. zu Eingriffen in Genstrukturen, geben zu können. ${ }^{151}$

145 Vgl. Preyer, G. (2018), S. 162-164; Staubmann, H. (2015), S. 291; Villányi, D. / Junge, M. / Brock, D. (2009), S. 371; Schimank, U. (2007), S. 244; Gerecke, U. (1998), S. 19.

146 Höffe, O. (2018), S. 7.

${ }^{147}$ Vgl. Bayertz, K. (2014), S. 146; Fenner, D. (2010), S. 13-15; Almond, B. (1995), S. 1-4; Singer, P. (1986), S. 1-2.

${ }^{148}$ Der Begriff selbst ist jedoch älter und lässt sich bis in die 1950er Jahre zurückverfolgen. Vgl. Stoecker, R. / Neuhäuser, C. / Raters, M.-L. (2011), S. 2.

${ }^{149}$ Vgl. Stoecker, R. / Neuhäuser, C. / Raters, M.-L. (2011), S. 2; Honnefelder, L. (1999), S. 273.

${ }^{150}$ Vgl. Winkler, E. R. / Coombs, J. R. (1993), S. 1.

${ }^{151}$ Vgl. Honnefelder, L. (1999), S. 275. 
Die Notwendigkeit, zusätzlich zur klassischen Ethik auch angewandte Ethiken zu betreiben, ergab sich folglich aus der Problematik, dass viele traditionelle Konzepte der Ethik nur sehr allgemeine Handlungsregeln zu geben vermögen ${ }^{152}$, welche jedoch nochmals in den spezifischen Problemkontexten ${ }^{153}$, die sich z. B. in der biomedizinischen Forschung (z. B. Menschenwürde) oder im Wirtschaftskontext (z. B. Wettbewerb) ergeben, differenzierter betrachtet werden müssen. So zeigt sich der Unterschied zu einer allgemeinen Ethik darin, dass diese das Handeln nach ethischen Maßstäben allgemein betrachtet, während eine angewandte bzw. Bereichsethik das Handeln in einem gewissen fachspezifischen Kontext analysiert. Ein zentrales Kennzeichen der angewandten Ethiken ist daher auch, dass eine fundierte ethische Analyse der betrachteten Gegenstände grundsätzlich bereichsspezifisches Wissen voraussetzt. ${ }^{154}$ In diesem Sinne subsumiert auch REICHELT: „Dieser Ruf verbindet sich seither mit der Forderung an die Ethik, nicht mehr nur theoretische, sondern vielmehr praktische Antworten auf die Herausforderungen der Gegenwart zu geben [...].“155 Ähnlich betont auch TETENS: „Mit allgemeinen Moralprinzipien sind wir nicht schon auf alle moralisch relevanten Situationen vorbereitet, vor die uns das Leben stellt." ${ }^{156}$ Auch NIDA-RUMELIN hebt die Relevanz einer spezifisch problemorientierten ethischen Forschung hervor und betont „daß für verschiedene Bereiche menschlicher Praxis unterschiedliche normative Kriterien angemessen sind, die sich [...] nicht auf ein einziges System moralischer Regeln und Prinzipien reduzieren lassen.“157 Während sich die allgemeine Ethik als ,reine“ Ethik folglich mit grundlegenden Fragen der Moral (bzw. des Ethos) beschäftigt, sind die Fragestellungen der angewandten Ethik themenspezifisch und problemorientiert.

Obschon sich in diesem Kontext in der Literatur ein dominierender Gebrauch des Terminus ,,angewandte Ethik“ feststellen lässt, finden sich auch weitere korrespondierende Bezeichnungen dieser Fachrichtung. So existiert in einigen Werken

\footnotetext{
152 Vgl. Fenner, D. (2010), S. 15-16.

153 Zum Überblick über die verschiedenen Themenfelder der angewandten Ethik vgl. Thurnherr, U. (2010).

154 Vgl. Fenner, D. (2010), S. 46.

155 Reichelt, M. (2018), S. 16.

156 Tetens, H. (2011), S. 21.

${ }^{157}$ Nida-Rümelin, J. (2005a), S. 63.
} 
auch die Bezeichnung „praktische Ethik“158, „konkrete Ethik“159, „Bereichsethik“160, ,Bindestrich-Ethik ${ }^{\star 161}$ oder ,problemorientierte Ethik ${ }^{\text {"162 }}{ }^{163}$ Dabei ist insbesondere der Begriff der praktischen bzw. angewandten Ethik einer intensiven Kritik unterzogen worden, welche von einer rein terminologischen Auseinandersetzung bis hin zur Infragestellung der Notwendigkeit einer speziell angewandten ethischen Forschung an sich reicht. ${ }^{164}$ In diesem Sinne argumentieren aus begrifflicher Perspektive z. B. manche Kritiker, dass der Begriff der angewandten Ethik eine Tautologie darstelle, da die Ethik immer schon eine angewandte, d. h. auf die menschliche Praxis bezogene Disziplin sei. ${ }^{165}$ So betont z. B. FISCHER, dass „die Rede von einer Angewandten Ethik als tautologisch erscheinen [muss]: Eine jede Ethik ist auf Anwendung hin entworfen [...]." ${ }^{166}$ Aus dieser Grundhaltung heraus plädiert beispielsweise NIDA-RUMELIN, statt des Terminus der angewandten Ethik, die Bezeichnung Bereichsethik zu verwenden, welche das Spezifische dieser ethischen Teildisziplin besser widerzuspiegeln vermöge.

Fasst man diese terminologischen Kontroversen zusammen, so lässt sich feststellen, dass der Begriff einer angewandten oder praktischen Ethik im begrifflich strengen Sinne möglicherweise zwar redundant erscheint. Dennoch ist in diesem Zusammenhang ersichtlich, dass sich der Terminus im Sinne einer bereichsspezifischen ethischen Untersuchung klar fachwissenschaftlich als Konvention durchgesetzt hat, ohne dass es je im Kern zu einer ernsthaften Konfusion hinsichtlich des Begriffsinhalts gekommen wäre. Zudem steht der Begriff in Referenz zur ebenfalls vorherrschenden angelsächsischen Bezeichnung applied ethics. Aus diesem Grunde soll auch im Rahmen dieser Arbeit der Terminus beibehalten

\footnotetext{
158 Vgl. z. B. Singer, P. (2011), S. 1.

159 Vgl. z. B. Pieper, A. (2017), S. 78; Siep, L. (2008), S. 47.

${ }^{160}$ Vgl. z. B. Nida-Rümelin, J. (2005a), S. 63.

161 Vgl. z. B. Dietzfelbinger, D. (2015), S. 38; Oermann, N. O. / Weinert, A. (2014), S. 67, welche die Bereichsethik synonym zum Terminus der Bindestrich-Ethik auffassen und diese als Unterkategorie der angewandten Ethik betrachten, sowie Rademacher, L. (2010), S. 284; Vieth, A. (2006), S. 55, welche ebenfalls den Begriff der Bereichsethik synonym zur Bindestrich-Ethik gebrauchen.

162 Vgl. z. B. Bayertz, K. (2014), S. 156.

${ }^{163}$ Vgl. zur Übersicht auch Reichelt, M. (2018), S. 21; Honnefelder, L. (2016), S. 644; Grübler, G. (2011), S. 303.

164 Vgl. Düwell, M. / Hübenthal, C. / Werner, M. H. (2011), S. 21; Fenner, D. (2010), S. 8-9.

165 Vgl. hierzu Schweidler, W. (2018), S. 11; Stoecker, R. / Neuhäuser, C. / Raters, M.-L. (2011), S. 3; Heinrichs, B. (2006), S. 113-114; Vieth, A. (2006), S. 19; Honnefelder, L. (1999), S. 273-274.

${ }^{166}$ Fischer, P. (2006), S. 14.
} 
werden, wobei im Weiteren der Fokus auf die Domäne der Wirtschaft gelegt wird. Hierbei lässt sich feststellen, dass gerade im deutschsprachigen Raum einige grundlegende konzeptionelle Arbeiten zur Wirtschafts- bzw. Unternehmensethik existieren. Da auch hier die Begriffsverwendung in der Literatur nicht vollständig konsistent vorgenommen wird, erfolgt im nachfolgenden Kapitel eine detaillierte Analyse der Begriffe der Wirtschafts- und Unternehmensethik, wobei auch die korrespondierenden englischen Termini der business- bzw. corporate ethics erörtert und zu den vorgenannten Begriffen in Verbindung gesetzt werden.

\subsection{Die drei Ebenen der Wirtschaftsethik und die Einordnung der Unternehmensethik}

Als besonders bedeutsames Teilgebiet der angewandten Ethik hat sich in den letzten Jahrzehnten im deutsch- wie auch englischsprachigen Raum die Wirtschaftsbzw. Unternehmensethik ${ }^{167}$ entwickelt. Dabei zeigt sich bereits in deutschsprachigen Veröffentlichungen, dass die Begriffe Wirtschafts- und Unternehmensethik nicht einheitlich verwendet werden, weshalb im Folgenden ein kurzer Literaturüberblick gegeben wird, bevor die grundlegenden und bis heute wirkmächtigen Konzepte der Wirtschaftsethik näher erörtert werden.

In der Literatur hat sich dabei eine klassische Dreiteilung ${ }^{168}$ wirtschaftsethischer Überlegungen entwickelt ${ }^{169}$, welche gerade im deutschsprachigen Raum

${ }^{167}$ Wichtig ist hierbei zu betonen, dass der Begriff der Wirtschaftsethik mehrdeutig ist. Neben der fachwissenschaftlichen Verwendung (Wirtschaftsethik als wissenschaftliche Disziplin), wie dieser auch im Rahmen dieser Arbeit zumeist Anwendung findet, kann der Terminus auch die Umsetzung in der Wirtschaftspraxis bezeichnen. Vgl. hierzu konzeptionell auch Enderle, G. (1996), S. 34, welcher dies auch für die business ethics betont und dieser zudem den Diskurs über wirtschaftsethische Fragen als begriffliche Interpretationsebene hinzufügt. Vgl. konzeptionell ähnlich auch De George, R. T. (2010), S. 9-17.

${ }^{168}$ Eine solche Differenzierung findet sich auch in wissenschaftstheoretischen Werken, so z. B. bei Brühl, R. (2017), S. 47-48 und in der Organisationssoziologie, z. B. bei Preisendörfer, P. (2016), S. 181; Sandhu, S. (2012), S. 87.

${ }^{169}$ Nach ZIMMERLI und AßLÄNDER stammt die Systematik ursprünglich aus dem amerikanischen Schrifttum (vgl. Zimmerli, W. C. / Aßländer, M. S. (2005), S. 322) und findet sich noch bei einigen klassischen amerikanischen Autoren der Wirtschaftsethik wieder (vgl. z. B. De George, R. T. (1987), S. 204; Matthews, J. B. / Goodpaster, K. E. / Nash, L. L. (1985), S. 509). In den aktuellen, weitverbreiteten Lehrbüchern der „,business ethics“ wird diese Differenzierung erkennbarerweise jedoch nicht mehr explizit fortgeführt. Vgl. hierzu ähnlich auch die Ausführungen in den aktuellen Auflagen bei De George, R. T. (2010); Goodpaster, K. E. / Nash, L. L. / De Bettignies, H.-C. (2006), welche diese Differenzierung ebenfalls nicht mehr ausführen. 
eine bis heute andauernde konzeptionelle Verbreitung gefunden hat. ${ }^{170}$ So können wirtschaftsethische Überlegungen in einem ersten Schritt auf die Gestaltung der wirtschaftlichen Rahmenordnung fokussieren (Makroebene). Darüber hinaus befassen sich wirtschaftsethische Betrachtungen auch insbesondere mit Unternehmen als korporativen Akteuren auf der Mesoebene. Dies hebt auch FENNER hervor: „Auf der Mesoebene hingegen fokussiert die Wirtschaftsethik ihr Augenmerk auf die Unternehmen als Organisationen oder Institutionen, die Träger von kollektiver Verantwortung sein können. "171 Schließlich stehen auf Mikroebene wirtschaftsethischer Überlegungen die handelnden Individuen im Fokus. Klassische Beispiele solcher Individualethiken umfassen beispielsweise die Konsumenten-, Produzenten- oder Investorenethik. ${ }^{172}$

Die Ebenen sind dabei nicht unabhängig voneinander, sondern durch intensive Verzahnung gekennzeichnet. So wirkt z. B. die geltende Rahmenordnung auf die Handlungen von Unternehmen wie auch Individuen ein, wobei Individuen wiederum in ihrem Handeln durch bewusste und unbewusste organisationsinterne Institutionen bzw. Organisationskulturen geprägt sind. In umgekehrter Richtung kann jedoch auch konstatiert werden, dass die untergeordneten Ebenen auf die übergeordneten Aggregate einwirken. So sind bspw. in freiheitlichen und partizipativen Gesellschaften Individuen in nicht unerheblichem Maße in die Gestaltung von Normen auf der staatlichen wie auch der organisationalen Ebene involviert und gerade Großkonzerne können durch Lobbyismus entscheidenden Einfluss auf die staatlichen und überstaatlichen Rahmensetzungstätigkeiten nehmen.

Wie diese drei klassischen Ebenen einer auf den Problembereich „Wirtschaft“ fokussierten angewandten Ethik jedoch genau zu benennen sind, differiert durchaus. In einer ersten, teilweise in der Literatur, so z. B. von HENTZE und THIES ${ }^{173}$ oder auch STEINMANN und LOHR ${ }^{174}$ gebrauchten Abgrenzung, bezeichnet der Terminus der Wirtschaftsethik lediglich die Makroebene, d. h. die Ebene der wirtschaftlichen Rahmenordnung. Wirtschaftsethik fokussiert folglich primär auf die Gestaltung der (v. a. staatlich definierten) Rahmennormen für Individuen wie auch Kollektive. Die darunterliegende Mesoethik wird dabei begriffstaxonomisch

${ }^{170}$ Vgl. Conrad, C. A. (2020), S. 15; Göbel, E. (2020), S. 99; Nietsch-Hach, C. (2016), S. 54; Renz, P. S. / Frischherz, B. / Wettstein, I. (2015), S. 3-4; Küpper, H.-U. (2011), S. 31; Neuhäuser, C. (2011c), S. 162; Jähnichen, T. (2008), S. 13; Pech, J. C. (2007), S. 47; Zimmerli, W. C. / Aßländer, M. S. (2005), S. 319.

${ }^{171}$ Fenner, D. (2010), S. 350.

172 Vgl. Göbel, E. (2020), S. 100-105.

173 Vgl. Hentze, J. / Thies, B. (2012), S. 53-73.

${ }^{174}$ Vgl. Steinmann, H. / Löhr, A. (1994a), S. 103-105 und S. 199. Vgl. zudem Steinmann, H. (2004), S. 105; Löhr, A. (1996), S. 51. 
als Unternehmensethik klassifiziert, während die ethischen Überlegungen auf der Mikroebene in der Regel als Individualethik bezeichnet werden. ${ }^{175}$

Wenngleich eine solche Differenzierung in der deutschsprachigen Literatur durchaus eine gewisse Rezeption erfahren hat, so erscheint ein zweiter ${ }^{176}$, noch weiter verbreiteter begrifflicher Zugang zu den drei Ebenen konzeptionell fruchtbarer. Denn grundsätzlich ist an der aufgezeigten Differenzierung problematisch, dass diese durch die Einengung der Bezeichnung Wirtschaftsethik auf die Makroebene keinen plausiblen Überbegriff mehr offeriert, welcher alle drei Ebenen einer auf den Problembereich der Wirtschaft ausgerichteten Ethik umfassen würde. ${ }^{177}$ Folglich ist es sinnvoll, in einem zweiten taxonomischen Verständnis, mit dem Begriff der Wirtschaftsethik nicht lediglich die kritische Analyse der wirtschaftlichen Rahmenordnung zu verbinden, sondern vielmehr „Wirtschaftsethik“ als Überbegriff für eine auf die Wirtschaft als Problemgegenstand fokussierte, angewandte Ethik zu erachten. Dieses Begriffsverständnis betont auch GÖBEL: „Wirtschaftsethik ist Anwendung der Ethik auf den Sachbereich Wirtschaft." 178 In dieser Taxonomie wird die Makroebene dann als Rahmenordnungs- bzw. Ordoethik bezeichnet, während, wie auch analog zur vorigen Begriffsauffassung, der Terminus der Unternehmensethik weiterhin auf die Mesoebene wirtschaftsethischer Überlegungen fokussiert und die Individualethik auf die im Wirtschaftskontext handelnden Individuen ${ }^{179}$ abzielt. ${ }^{180}$ Einer solchen begrifflichen Auffassung soll im Rahmen dieser Arbeit gefolgt werden. Die

175 Vgl. auch Dietzfelbinger, D. (2015), S. 16; Clausen, A. (2009), S. 26-27; Gerum, E. / Mölls, S. (2009), S. 304; Preuss, L. (1999), S. 407; Kreikebaum, H. (1996a), S. 14; Enderle, G. (1991), S. 180 sowie implizit durch den Titel auch bei Aaken, D. v. / Schreck, P. (2015). ${ }^{176}$ In diesem Sinne betont auch PANTHER: „Der Begriff ,Wirtschaftsethik“ scheint in zwei Bedeutungen verwendet zu werden: Einmal umfassend, zur Bezeichnung einer Ethik des funktional ausdifferenzierten Bereichs Wirtschaft, zum anderen als Ethik der Wirtschaftsordnung." (Panther, S. (2005), S. 67).

177 So muss auch DIETZFELBINGER als Überbegriff auf die Bezeichnung „Ethik der Ökonomie“ (Dietzfelbinger, D. (2015), S. 16) ausweichen.

178 Göbel, E. (2020), S. 99, in der Quelle fett herv.

${ }^{179}$ Teilweise wird in der Literatur auch eine konzeptionelle Einengung auf Führungskräfte bzw. Manager vorgenommen, so dass in diesem Kontext dann der Begriff der Managementoder Führungsethik gebraucht wird. Vgl. z. B. Neuhäuser, C. (2011c), S. 164.

180 Vgl. Göbel, E. (2020), S. 99-116; Ulrich, P. (2016a), S. 309-311; Noll, B. (2013), S. 4445; Küpper, H.-U. / Sandner, K. (2011), S. 29-31; Neuhäuser, C. (2011c), S. 162; Fenner, D. (2010), S. 350-351. Vgl. auch Zimmerli, W. C. / Aßländer, M. S. (2005), S. 322, welche hervorheben, dass die Makroebene auch als „Wirtschaftsethik im engeren Sinne“ aufgefasst werden kann. 
vorausgegangenen Überlegungen, insbesondere auch zur gegenseitigen Beeinflussung der wirtschaftsethischen Ebenen, verdeutlicht nochmals die folgende Abbildung 3.6.

Abbildung 3.6 Die drei Ebenen der Wirtschaftsethik und ihre gegenseitige Beeinflussung ${ }^{181}$

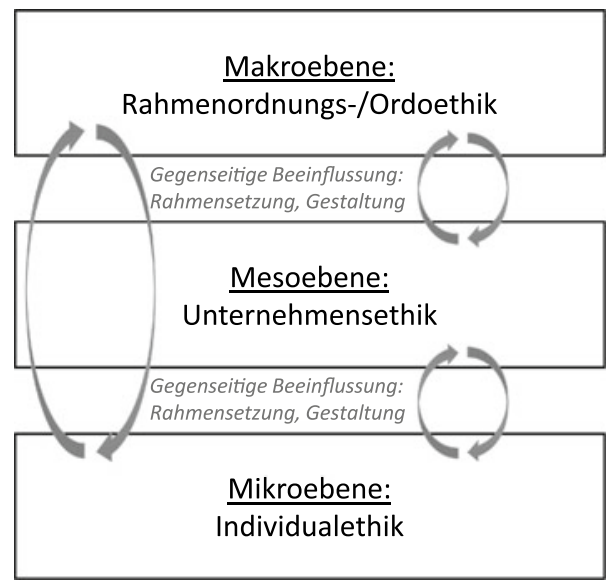

Betrachtet man den angelsächsischen Bereich, so erscheint in einem ersten Schritt der in diesem Kontext häufig gebrauchte Begriff der business ethics ${ }^{182}$ in etwa dem deutschen Terminus der Wirtschaftsethik zu entsprechen, also das gesamte Feld der wirtschaftsethischen Forschung sowie auch die Einbindung ethischer Überlegungen in die Wirtschaftspraxis zu bezeichnen. Eine intensivere Auseinandersetzung legt jedoch dar, wie auch die späteren Ausführungen noch detaillierter zeigen, dass die angelsächsische Forschung nicht nur eher pragmatisch orientiert ist und weniger auf grundlegende konzeptionelle Fragen fokussiert, sondern zudem auch die strikte Trennung in die drei Ebenen der Wirtschaftsethik in der gegenwärtigen Forschung dort eher unüblich ist. So zeigt die Forschungspraxis der business ethics, dass diese im Vergleich zum obigen deutschsprachigen Verständnis zumeist in einem engeren Sinne auf Unternehmen und insbesondere sehr stark individualethisch auf das Managerhandeln in

181 Vgl. grundlegend auch Göbel, E. (2020), S. 116.

182 Vgl. Matten, D. / Palazzo, G. (2008), S. 51. 
Unternehmen abzielt ${ }^{183}$, so dass z. B. ordoethischen Überlegungen ein deutlich geringerer Stellenwert zukommt. ${ }^{184}$ Diese Ausrichtung mag gerade auch die Schwierigkeiten erklären, mit welchen deutschsprachige Autoren in der Übersetzung ihrer Werke konfrontiert sind. ${ }^{185}$ Um eine vergleichbare Differenzierung zu erzielen, werden dabei in Übersetzungen häufig verwandte, aber im Angelsächsischen eher weniger geläufige, Begrifflichkeiten wie corporate ethics und economic ethics genutzt, um eine ähnliche Systematisierung wie in der deutschsprachigen Forschung zu erzielen. Das genaue Begriffsverständnis differiert jedoch in der Übersetzung der ursprünglich deutschsprachigen Literatur. So wird beispielsweise in der englischen Übersetzung des Werkes von PETER UlRICH Wirtschaftsethik im integrativen Sinne als ,economic ethics“ bezeichnet, andere wirtschaftsethische Ansätze allerdings als „,business ethics“, während „,corporate ethics“186 wiederum als Übersetzung der Bezeichnung „Unternehmensethik“ genutzt wird. ${ }^{187}$ Diese Übersetzung folgt jedoch keinem übergreifenden Konsens. So gebraucht beispielsweise LUTTGE die Bezeichnung „economic ethics“ zwar ebenfalls als wirtschaftsethischen Überbegriff, nutzt allerdings wiederum den Begriff der business ethics ${ }^{188}$ im Sinne von „Unternehmensethik“. ${ }^{189}$ Obschon der Begriff der economic ethics als Übersetzung des deutschen Wirtschaftsethikbegriffs durchaus eine gewisse Verbreitung gefunden hat ${ }^{190}$, so muss doch konstatiert werden, dass dieser nach wie vor im englischsprachigen Schrifttum eine eher geringe Verwendung ${ }^{191}$ findet. Aus diesem Grund soll im Weiteren

\footnotetext{
${ }^{183}$ Vgl. Nietsch-Hach, C. (2016), S. 53; Heidenreich, F. (2012), S. 89.

184 In diesem Sinne betonen auch STEINMANN und LOHR: ,The institutional segmentation of the debate has also led to the fact that the discussion of the field in Germany doesn't strictly correspond to the international term ,business ethics'" (Steinmann, H. / Löhr, A. (1996), S. 23, in der Quelle ebenfalls herv.).

185 So hebt auch der Übersetzer des Werkes „Integrative Wirtschaftsethik“ von PETER ULRICH ins Englische, JAMES FEARNS, hervor: ,A number of concepts frequently used in the German literature on economic ethics have no direct equivalent in English or carry connotations and associations which may not be immediately evident to an English reader." (Ulrich, P. (2008a), S. XII).

186 Vgl. zu dieser Begriffsauffassung auch Enderle, G. (1996), S. 40.

187 Vgl. Ulrich, P. (2008a), S. XII. Vgl. auch Ulrich, P. (2000), S. 42.

188 Diesem Begriffsverständnis folgt z. B. auch die Übersetzung bei De George, R. T. (1992).

${ }^{189}$ Vgl. Lütge, C. (2005), S. 113.

${ }^{190}$ Vgl. terminologisch z. B. auch Dierksmeier, C. (2016).

${ }^{191}$ Vgl. zu einigen hervorzuhebenden Ausnahmen, z. B. Hole, J. (2016); Barrera, A. (2007); Graafland, J. J. (2006); Rich, A. (2006). Diese stammen jedoch vielfach aus dem Kontext des europäischen Diskurses. Vgl. auch Cowton, C. J. / Haase, M. (2008), welche ,economic-“ und „,business ethics“ relativ synonym gebrauchen sowie DeMartino, G. F. / McCloskey, D.
} 
business ethics im obigen aufgezeigten Sinne als Überbegriff für Wirtschaftsethik $^{192}$ und corporate ethics im Sinne einer auf die Mesoebene bezogenen Wirtschaftsethik, d. h. als Unternehmensethik, aufgefasst werden.

Nachdem nun die Grundlagen hinsichtlich der drei Ebenen der Wirtschaftsethik erarbeitet wurden, stellt sich die Frage, welche wirtschaftsethischen Konzeptionen im Laufe der Zeit entwickelt wurden. Daher werden im Folgenden die zentralen und bis heute wirkmächtigen deutsch- wie auch englischsprachigen Konzeptionen vorgestellt und abschließend einer kritischen Würdigung unterzogen.

\subsection{Zentrale deutschsprachige Konzeptionen}

\subsection{1 Überblick}

Eine Betrachtung der Entwicklung deutschsprachiger Wirtschaftsethikkonzeptionen sowie des aufgezeigten wissenschaftstheoretischen Diskurses um die Notwendigkeit bzw. Zulässigkeit von Werturteilen in der betriebswirtschaftlichen Forschung zeigt, dass bis in die 1970er Jahre hinein originär wirtschaftsethische Überlegungen im betriebswirtschaftlichen Kontext kaum repräsentiert waren bzw. lediglich als „Außenseitermeinungen“193 aufgefasst wurden. Folglich existierten bis zu Beginn der 1980er Jahre kaum eigenständige wirtschaftsethische Konzeptionen im deutschsprachigen Raum. ${ }^{194}$ Seit dieser Zeit, und insbesondere seit Mitte der 1980er und dem Beginn der 1990er Jahre, findet jedoch eine verstärkte konzeptionelle Beschäftigung mit wirtschaftsethischen Fragestellungen statt. ${ }^{195}$ Wie die weiteren Ausführungen zeigen, ist, analog zu den bereits diskutierten Spezifika der betriebswirtschaftlichen Grundlagenforschung, auch für die deutschsprachige Wirtschaftsethik eine umfangreiche konzeptionelle Auseinandersetzung prägnant, welche mit einem intensiven und bis heute anhaltenden

N. (2016), welche wiederum ,economic ethics“ im Rahmen einer Berufsethik für Ökonomen diskutieren.

192 So konstatiert auch KUPPER: „Im englischen Sprachgebrauch hat sich für den Begriff der Wirtschaftsethik die Bezeichnung ,Business Ethics' weitgehend durchgesetzt.“ (Küpper, H.U. (2011), S. 31).

193 Steinmann, H. / Löhr, A. (2002a), S. 515.

${ }^{194}$ Vgl. Steinmann, H. / Löhr, A. (2002a), S. 515.

195 Vgl. Küpper, H.-U. (2011), S. 107; Steinmann, H. / Löhr, A. (2002b), S. 95; Steinmann, H. / Kustermann, B. (1996), S. 12; Kaufmann, F.-X. (1992), S. 20. 
Diskurs über die „richtige“ wirtschaftsethische Konzeption korrespondiert. In diesem Sinne betont auch PREUSS den ,wide scope business ethics has reached in German speaking countries; works which in their majority are not yet available in English translation. [...] Although business ethics is a relatively new field, the scope of ethical theory in the German academic literature has reached considerable dimensions, both in quantity and quality." 196

Die Gründe, welche zu einer intensiveren Auseinandersetzung mit wirtschaftsethischen Fragestellungen im deutschsprachigen Raum führten, sind dabei vielfältig. ${ }^{197}$ Neben einem bereits in den 1970er Jahren gestiegenen Umweltbewusstsein der Bevölkerung ${ }^{198}$ kann auch eine zunehmende Einforderung unternehmerischer Verantwortung seitens der Bevölkerung im Zuge diverser Unternehmensskandale in den 1980er Jahren ${ }^{199}$ konstatiert werden. In diesem Sinne betont auch STEINMANN: „Unternehmensethik ist keine Erfindung von Akademikern, die nach einem neuen Betätigungsfeld suchen. Der Ruf nach einer ethischen Orientierung betriebswirtschaftlichen Handelns ist vielmehr über die Jahre hin aus der praktischen Erfahrung vieler Skandale entstanden [...]." ${ }^{200}$ Dabei lassen sich auch eine zunehmend kritische Bevölkerung und eine intensive Diskussion feststellen, wie STEINMANN und LOHR hervorheben: „As far as practice within companies is concerned, the public has become more sensitive to questions of Business Ethics through a number of serious scandals. [...] The topic of Business Ethics has gained greater popularity through massive coverage by the mass-media. ${ }^{\text {201 }}$

Insbesondere seit Mitte der 1980er sowie dem Beginn der 1990er Jahre folgte auch die deutschsprachige Forschung der Entwicklung im angelsächsischen Raum

\footnotetext{
196 Preuss, L. (1999), S. 407 und S. 417.

197 Vgl. zur Übersicht auch Lenk, H. / Maring, M. (1996), S. 1-2.

198 Vgl. Koslowski, P. (1990a), S. 34.

${ }^{199}$ Vgl. Steinmann, H. / Löhr, A. (2002a), S. 513; Löhr, A. (1996), S. 49; Steinmann, H. / Löhr, A. (1988), S. 299.

200 Steinmann, H. (2004), S. 106, in der Quelle ebenfalls herv. Vgl. ähnlich auch Steinmann, H. / Löhr, A. (2002b), S. 100. Ähnliches gilt, wenngleich historisch bereits früher, für den angelsächsischen Kontext. Vgl. hierzu auch Vogel, D. (1992), S. 31-34.

${ }^{201}$ Steinmann, H. / Löhr, A. (1992), S. 140, in der Quelle ebenfalls herv.
} 
mit einer Vielzahl ${ }^{202}$ an detailliert ausgearbeiteten Konzeptionen. ${ }^{203}$ Im Weiteren werden nun die bis heute wirkmächtigsten Positionen vorgestellt. Unter diesen werden zum Teil nochmals bedeutsame Subkonzeptionen der Literatur erarbeitet. Häufig wird hierbei klassisch zwischen den drei zentralen Ethikkonzeptionen von SteinmanN und LOHR, HomanN und seinen Schülern sowie PeTER UlRich differenziert. ${ }^{204}$ Diese zentralen Strömungen werden im Weiteren konzeptionell detaillierter vorgestellt, bevor weitere, bis heute wirkmächtige Ansätze erörtert werden. Diese Überlegungen fasst nochmals die nachfolgende Abbildung 3.7 zusammen.

Darüber hinaus existieren auch weitere bekannte Autoren der deutschsprachigen Wirtschaftsethik, welche jedoch nicht im Detail im Rahmen dieser Arbeit diskutiert werden können. Neben den bereits einführend aufgezeigten normativen Strömungen der BWL sowie einigen klassischen Arbeiten sozialethischer Autoren wie beispielsweise VON NELL-BREUNING ${ }^{205}$, kann insbesondere auch KosLOWSKI genannt werden, welcher sich schon zu Beginn der 1980er Jahre intensiv mit originär wirtschaftsethischen Fragestellungen befasste. KOSLOWSKI hat dabei jedoch, ähnlich wie auch in den meisten angelsächsischen Werken, keine umfassende und eigenständige Ethikkonzeption entwickelt,

202 So bemerken auch STEINMANN und LOHR pointiert: „Especially in Germany, there has been a flood of publications since the late 1980 s, which has led to a very distinctive discussion of ethics, economics, and business [...]. This literature is actually built on a long term tradition of research in the field of ethics and business in Germany throughout the whole of this century." (Steinmann, H. / Löhr, A. (1996), S. 22, in der Quelle ebenfalls herv.). Wichtig erscheint in diesem Zusammenhang jedoch nochmals hervorzuheben, dass hierbei der gesamte deutschsprachige Raum miteinbezogen werden muss. Vgl. hierzu auch Preuss, L. (1999), S. 407.

${ }^{203}$ Einen umfassenden Überblick, insbesondere aus unternehmensethischer Perspektive, gibt dabei Küpper, H.-U. (2011), S. 107. In diesem Sinne betont auch KUPPER: „Inzwischen ist auf dem Weg zur Anerkennung der Unternehmensethik in der BWL einiges erreicht. Zuerst vorsichtig seit 1990 und deutlicher nach 2000 gibt es in ihr eine intensivere Auseinandersetzung mit unternehmensethischen Konzepten und Problemen.“ (Küpper, H.-U. (2015), S. 317).

${ }^{204}$ Vgl. Göbel, E. (2020), S. 83-96; Ulrich, P. (2016a), S. 103-135; Löhr, A. (2004), Sp. 1515-1516; Ulrich, P. (1989), S. 21. Vgl. auch die Ausführungen bei Neuhäuser, C. (2011c), S. 161, welcher zusätzlich viertens die Governanceethik nach WIELAND hervorhebt. Vgl. auch die Klassifikationen bei Holzmann, R. (2019), S. 72; Heidenreich, F. (2012), S. 52, welche jedoch nur die Konzeptionen von UlRICH, HOMANN sowie WIELAND betonen.

205 Vgl. z. B. die Überlegungen in Nell-Breuning, O. v. (1987); Nell-Breuning, O. v. (1975); Nell-Breuning, O. v. (1960). 


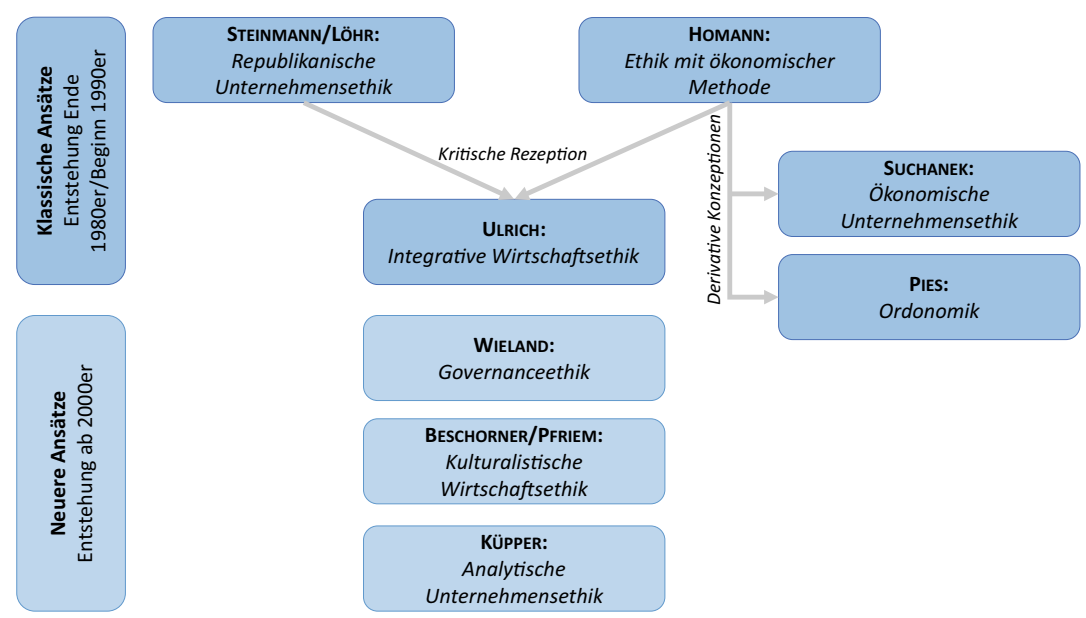

Abbildung 3.7 Zentrale Wirtschaftsethikkonzeptionen des deutschsprachigen Raums

sondern wendet vielmehr, ähnlich wie auch BowIE oder SISON ${ }^{206}$, deontologische oder tugendethische Konzepte auf wirtschaftliche Fragestellungen $a^{207}$, wobei bei KOSLOWSKI vielfach ein Fokus auf volkswirtschaftlichen Überlegungen liegt. ${ }^{208}$ Gleiches gilt auch für die relativ neuen Beiträge von GESANG, welcher wirtschaftsethische Fragen auf der globalen Ebene durch ein utilitaristisches Kalkül betrachtet. ${ }^{209}$ Schließlich sei mit den Arbeiten KREIKEBAUMS auch eine dezidiert theologisch fundierte Konzeption genannt, welche sich aus einer christlichen Perspektive mit entscheidungsethischen Fragen beschäftigt. ${ }^{210}$

\footnotetext{
206 Vgl. hierzu die Ausführungen in Abschnitt 3.5.

${ }^{207}$ Vgl. z. B. Koslowski, P. (1988), S. 43-44.

${ }^{208}$ Vgl. z. B. die klassischen Ausführungen in Koslowski, P. (1998); Koslowski, P. (1994); Koslowski, P. (1992); Koslowski, P. (1990a); Koslowski, P. (1990b).

${ }^{209}$ Vgl. Gesang, B. (2016), S. 34-37.

${ }^{210}$ Vgl. konzeptionell z. B. Kreikebaum, H. (1996a), S. 249-264 sowie zur christlichen Prägung der Konzeption Kreikebaum, H. (1996b), S. 119; Kreikebaum, H. (1991), S. 219.
} 


\subsubsection{Die republikanische Ethik Steinmanns und LöHRS}

Eine der frühesten und bis heute verbreitetsten Wirtschaftsethikkonzeptionen stammt von STEINMANN und LÖHR, welche vor allem auf die wirtschaftsethische Mesoebene (Unternehmensethik) fokussieren. Zentrales Ziel der Konzeption ist hierbei die gesellschaftliche Friedenssicherung als oberste Maxime. ${ }^{211}$ In diesem Zusammenhang kritisieren STEINMANN und LÖHR die unkritische Übernahme des ökonomischen Prinzips im Wirtschaftskontext und insbesondere auch die unreflektierte Unterstellung, dass das Gewinnprinzip gesellschaftlich grundsätzlich legitimiert sei. Vielmehr repräsentiere gerade das Gewinnstreben kein bedingungslos akzeptiertes, sondern vielmehr ein bedingtes Ziel, welches stets unter dem gesellschaftlichen Vorbehalt der Legitimität stehen muss. ${ }^{212}$ Ihre Ethikkonzeption beruht dabei auf der durch die „Lebenserfahrung gewonnene[n] Einsicht, daß Ethik um des Friedens willen in Gang gebracht werden muß [...].“213 In diesem Sinne sei „das Gewinnprinzip in der Marktwirtschaft immer unter Friedensvorbehalt ${ }^{\star 214}$ anzuwenden, wodurch das Management aufgefordert ist, sich konstant um die gesellschaftliche Legitimität des erwerbswirtschaftlichen Strebens zu bemühen, wobei insbesondere auch stets kritisch die Mittel zu prüfen sind, mit welchen die unternehmerischen Gewinne erzielt werden. ${ }^{215}$ Fasst man diese Überlegungen zusammen, so kommt man zur folgenden Definition der Konzeption nach STEINMANN und LÖHR: „Unternehmensethik umfaßt alle durch dialogische ${ }^{216}$ Verständigung mit den Betroffenen begründeten bzw. begründbaren materialen und prozessualen Normen, die von einer Unternehmung zum

\footnotetext{
${ }^{211}$ Vgl. Steinmann, H. / Löhr, A. (2015), S. 269; Steinmann, H. (2011), S. 101; Steinmann, H. (2008b), S. 135; Steinmann, H. / Löhr, A. (1997), S. 23; Steinmann, H. / Löhr, A. (1995a), S. 143; Steinmann, H. / Löhr, A. (1994a), S. 76.

212 Vgl. Steinmann, H. / Löhr, A. (2015), S. 292.

213 Steinmann, H. / Löhr, A. (1994a), S. 76. Vgl. hierzu auch Löhr, A. (1996), S. 57.

214 Steinmann, H. / Löhr, A. (2015), S. 292, in der Quelle ebenfalls herv. Vgl. auch Steinmann, H. / Löhr, A. (1997), S. 30.

215 Vgl. Steinmann, H. / Löhr, A. (2015), S. 269-271; Steinmann, H. (2004), S. 106; Steinmann, H. / Löhr, A. (1988), S. 308-309; Steinmann, H. / Löhr, A. (1987), S. 454-455.

${ }^{216}$ STEINMANN und LÖHR verwenden die Termini „Dialog“ und „Diskurs“ weitgehend synonym und bezeichnen ihre Konzeption folglich sowohl als Dialog-, aber auch als Diskursethik. Vgl. Steinmann, H. / Löhr, A. (2015), S. 269; Steinmann, H. (2011), S. 101; Steinmann, H. (2008b), S. 137; Löhr, A. (2004), Sp. 1514.
} 
Zwecke der Selbstbindung verbindlich in Kraft gesetzt werden, um die konfliktrelevanten Auswirkungen des Gewinnprinzips bei der Steuerung der konkreten Unternehmensaktivitäten zu begrenzen.“"217

Ihre Konzeption beruht wesentlich auf den diskursethischen Überlegungen von APEL und HABERMAS, welche ethisch die Notwendigkeit des Einbezugs aller Betroffenen in einen Diskurs fordern, da nicht abstrakt oder monologisch ${ }^{218}$, sondern nur in einem herrschaftsfreien Diskurs die Legitimität von Handlungen oder Entscheidungen geprüft werden kann. Wie STEINMANN und LÖHR betonen, ist neben der Zwanglosigkeit auch die Unvoreingenommenheit sowie auch die Sachverständigkeit der Teilnehmer von zentraler Bedeutung. ${ }^{219}$ In diesem Sinne gilt mit HABERMAS folglich das Ideal des ,Zwanglosen Zwang[s]“220 des besseren Arguments. Dabei unterscheiden STEINMANN und LÖHR zudem zwischen Argumentation und Appellen, welches auch für die weiteren Ausführungen dieser Arbeit fruchtbar erscheint. So gilt im Gegensatz zur diskursiven Argumentation, dass im Rahmen von Appellen „der Redende seine eigene Subjektivität nicht zur Disposition [stellt]. Das Redeziel ist nicht die wechselseitige Überzeugung, sondern die geschickte Überredung des Gegenüber [sic!] [...].“221 Dagegen erscheint aus legitimatorischer Perspektive gerade der freie Diskurs ${ }^{222}$ elementar, um sicherzustellen, dass das unternehmerische Erwerbsstreben stets nur mit legitimen Mitteln realisiert wird. In diesem Kontext heben die Autoren hervor, dass

${ }^{217}$ Steinmann, H. / Löhr, A. (1991a), S. 10. Vgl. ähnlich auch Steinmann, H. / Löhr, A. (1997), S. 33; Steinmann, H. / Löhr, A. (1996), S. 50; Steinmann, H. / Löhr, A. (1989), S. 106; Steinmann, H. / Löhr, A. (1988), S. 310; Steinmann, H. / Oppenrieder, B. (1985), S. 173-174. ${ }^{218}$ Vgl. hierzu auch Schreyögg, G. / Koch, J. (2020), S. 105-110; Steinmann, H. (2008a), S. 347; Steinmann, H. / Scherer, A. G. (2000), S. 170.

219 Ähnlich wie auch in der klassischen diskursethischen Literatur (vgl. z. B. Habermas, J. (2015), S. 161-162; Apel, K.-O. (1992), S. 34-37) heben auch STEINMANN und LÖHR hervor, dass diese Annahmen Idealbedingungen repräsentieren, welche in der Realität nie vollständig zu erreichen, aber dennoch als Richtlinie zur Bewertung realer Diskurse von Bedeutung seien (vgl. Steinmann, H. / Löhr, A. (1994a), S. 78-81). Vgl. für eine Zusammenstellung zentraler Eigenschaften eines idealen Diskurses auch Ulrich, P. (1981), S. 68.

${ }^{220}$ Habermas, J. (2015), S. 123.

${ }^{221}$ Steinmann, H. / Löhr, A. (1994a), S. 77.

222 In diesem Sinne betont auch BESCHORNER: ,[D]iscourse-ethical approaches can be called upon for justifying moral principles." (Beschorner, T. (2006), S. 127). 
ein solcher Diskurs stets auf dem Austausch und der Prüfung guter Gründe basieren muss und keinesfalls auf der Ausübung von Macht. ${ }^{223}$ Folglich postulieren STEINMANN und LOHR: „Konflikte mit den von unternehmerischen Entscheidungen Betroffenen (Stakeholdern) sollen im Grundsatz friedlich, das heißt durch argumentative Verständigung statt durch Machtgebrauch [...] beigelegt werden." ${ }^{224}$ Dabei steht stets das Gemeinwohl (res publica) ${ }^{225}$ im Fokus, welches den Entscheidungsträger in seinen Handlungen zu leiten habe und zugleich der Konzeption auch ihre Bezeichnung als ,republikanische Ethik“226 verleiht. In diesem Sinne fordern STEINMANN und LÖHR: „Privatunternehmer bleiben immer Republikaner, d. h. der öffentlichen Sache verpflichtet.“227

Zusätzlich intendiert die Unternehmensethik von STEINMANN und LÖHR eine Transzendierung rein legalistischen Denkens, welches lediglich als grundlegendes normatives Fundament aufgefasst werden könne: „Diese begrifflichen Präzisierungen machen insgesamt deutlich: die Unternehmensethik fordert sowohl zu einer situationsgerechten verantwortlichen Anwendung des Gewinnprinzips auf, wie auch zu einer kritisch-loyalen Ergänzung des Rechts.“228 Hinsichtlich der konkreten, handlungsleitenden Normen betonen STEINMANN und LOHR aber auch die Grenzen der einzelstaatlichen Regulierung 229 in der globalisierten Wirtschaftswelt, in welcher die Staaten alleinig kaum mehr als dominante normsetzende Akteure auftreten (können). Gerade aus dieser Perspektive entsteht folglich die Notwendigkeit, als Unternehmen selbst Verantwortung zu übernehmen, ,nicht zuletzt deshalb, weil ein Weltgesetzgeber für globale Konfliktregelungen nicht

${ }^{223}$ Der Begriff der Macht ist überaus schillernd. Eine klassische sozialwissenschaftliche Definition stammt von MAX WEBER, welcher Macht als ,,jede Chance, innerhalb einer sozialen Beziehung den eigenen Willen auch gegen Widerstreben durchzusetzen, gleichviel worauf diese Chance beruht" (Weber, M. (1976), S. 28) definierte.

${ }^{224}$ Steinmann, H. / Löhr, A. (2015), S. 269, in der Quelle ebenfalls herv. Vgl. ähnlich auch Steinmann, H. (2008b): „Peaceful resolution of conflicts does not result from the use of power (in its manifold forms) [...].“ (S. 135).

${ }^{225}$ Vgl. Steinmann, H. / Löhr, A. (2015), S. 269; Steinmann, H. (2008b), S. 143-144; Steinmann, H. / Löhr, A. (1997), S. 33; Steinmann, H. / Löhr, A. (1996), S. 49; Steinmann, H. / Löhr, A. (1994b), S. 148.

${ }^{226}$ Die Bezeichnung beruht dabei ursprünglich auf den Überlegungen von Lorenzen, P. (1991), S. 50 und S. 62-63. Vgl. hierzu auch Steinmann, H. / Löhr, A. (1997), S. 33.

227 Steinmann, H. / Löhr, A. (1994a), S. 96.

228 Steinmann, H. / Löhr, A. (1994a), S. 118. Vgl. hierzu auch Steinmann, H. (2008a), S. 339; Steinmann, H. / Löhr, A. (1995b), S. 227.

${ }^{229}$ Vgl. Steinmann, H. (2011), S. 102; Steinmann, H. (2008a), S. 339; Steinmann, H. / Oppenrieder, B. (1985), S. 173. 
existiert.“230 Dies gilt insbesondere, da viele Großkonzerne nicht nur in nicht unerheblichem Maße an staatlicher oder überstaatlicher Rahmenordnungssetzung partizipieren, sondern auch durch freiwillige Selbstbeschränkungen umfangreiche Eigenregulierung auf globaler Ebene betreiben: „Empirische Untersuchungen zeigen hier, dass es oft gerade Akteure aus der Privatwirtschaft sind, welche mangels eines Weltgesetzgebers die Initiative für selbstdisziplinierende transnationale Regulierungen ergreifen, neben Konsumenten- und Umweltgruppen.“231 Diese Perspektive wird auch im angelsächsischen Bereich geteilt. So heben ebenso CRANE und MATTEN hervor, dass gerade große, global tätige Unternehmen nicht unerheblich an der weltweiten Gestaltung von Wirtschaftsnormen beteiligt sind: ,[W]hat we have also increasingly witnessed is business itself becoming more involved in standard setting and enforcement, through self-regulation, global codes of conduct, ethical sourcing and so on [...]." ${ }^{\text {232 }}$ In diesem Sinne ist daher die Unternehmensführung stets aufgerufen, sich für eine Verbesserung der globalen Wirtschaftsordnung mit größerer Teilhabe und Einflussnahme aller Stakeholdergruppen einzusetzen. So fordere die unternehmensethische Konzeption nach STEINMANN und LOHR zwar ,primär subsidiäre Lösungen; wo dies aber nicht möglich ist, gehört es zur unternehmerischen Pflicht, Lösungsversuche auf übergeordneten Regelungsebenen in Gang zu bringen." ${ }^{233}$

Fasst man die Überlegungen von STEINMANN und LÖHR zusammen, so ergibt sich ein starker Fokus auf die kritische Würdigung der Mittel, welche dem erwerbswirtschaftlichen Streben ${ }^{234}$ zugrunde liegen. Diese Mittel müssen unter diskursivem Einbezug (idealtypisch) aller Stakeholder einer kritischen Prüfung unterzogen werden, wobei die Unternehmensführung dazu angehalten ist, keine Gewinne anzustreben, welche als illegitim aufgefasst werden würden. Aus diesem Grunde sollte sich das Unternehmen als good corporate citizen im Sinne des gesellschaftlichen Friedens selbst in seinem Gewinnstreben beschränken und auf einen illegitimen Gewinnanteil verzichten. In diesem Sinne fordert die

\footnotetext{
${ }^{230}$ Steinmann, H. (2011), S. 100.

${ }^{231}$ Steinmann, H. (2004), S. 105-106, in der Quelle ebenfalls herv.

${ }^{232}$ Crane, A. / Matten, D. (2004), S. 362-363. Vgl. auch Wettstein, F. / Waddock, S. (2005), S. 304.

${ }^{233}$ Steinmann, H. / Löhr, A. (1994a), S. 108.

234 Bei STEINMANN und LÖHR als „Gewinnstreben“ aufgefasst. Es sei nochmals angemerkt, dass im Rahmen dieser Arbeit, wie einleitend bereits ausgeführt wurde, Unternehmen durch den Fokus auf Wertschöpfung als erwerbswirtschaftliches Prinzip aufgefasst werden. Vgl. hierzu die Ausführungen in Abschnitt 2.6.4.2.
} 
Konzeption nach STEINMANN und LÖHR folglich eine kritisch reflektierte bzw. „situationsgerechte Anwendung des Gewinnprinzips. “235

Obschon die grundlegenden Überlegungen der Konzeption von STEINMANN und LÖHR in der Literatur eine breite Rezeption erfahren haben ${ }^{236}$, hat sich an der Auffassung, Ethik könne als Selbstbeschränkung des Gewinnstrebens aufgefasst werden, auch deutliche Kritik formuliert, wobei kritische Stimmen einen solchen Ansatz als ,praxisorientiertes Kompensationsmodell“237, „Unterdrückungsmodell“238 oder gar als „,Reparaturethik“239 bezeichnen, welcher konzeptionell einer moralisch defizitären Wirtschaftspraxis gegenübergestellt werde. $^{240}$ Eine dieser kritischen Positionen stammt von HOMANN und seinen Schülern, welche im Folgenden näher erörtert wird.

\subsubsection{Die Rahmenordnungsethik Homanns}

\subsubsection{Konzeptionelle Grundlagen}

Einen anderen Zugang zur Wirtschaftsethik nimmt HOMANN ein. Er positioniert seine Konzeption explizit gegen Ansätze, wie z. B. von STEINMANN und LÖHR ${ }^{241}$, welche er als rein ,appellativ“ und damit für die wirtschaftliche Praxis als zu wenig überzeugend kritisiert. Zwar heben STEINMANN und LOHR gerade hervor, gegenüber den Stakeholdern nicht appellativ, sondern diskursiv argumentierend zu verfahren, die Forderung selbst jedoch, dass das Unternehmen zum Erhalt des gesellschaftlichen Friedens freiwillig auf Gewinne verzichten, sich also selbst restringieren sollte, bleibt freilich ein normatives Postulat, bzw. ein an die Unternehmensführung gerichteter Appell, wobei sich nach HOMANN die praktische Frage stellt, wie dies gegenüber dem Unternehmen überzeugend $\mathrm{zu}$ begründen wäre.

${ }^{235}$ Steinmann, H. / Löhr, A. (1994a), S. 107, in der Quelle fett herv. Vgl. ähnlich auch Löhr, A. (1991), S. 234; Steinmann, H. / Löhr, A. (1988), S. 308.

${ }^{236}$ So begreifen auch GERUM und MöLLS Unternehmensethik explizit als „Begrenzung des Gewinnprinzips" (Gerum, E. / Mölls, S. (2009), S. 303, in der Quelle fett herv.) in Situationen, in welchen andernfalls unmoralisch gehandelt würde.

${ }^{237}$ Ulrich, P. (2016a), S. 103, in der Quelle ebenfalls herv.

${ }^{238}$ Meran, J. (1987), S. 28, in der Quelle unterstrichen herv.

${ }^{239}$ Mittelstrass, J. (1991), S. 104-105.

${ }^{240}$ Vgl. Göbel, E. (2020), S. 83-84.

241 Ähnlich kritisch erachtet HOMANN auch die Konzeption von UlRICH. Vgl. hierzu Homann, K. (1994b), S. 122; Homann, K. / Blome-Drees, F. (1992), S. 179-185. 
Grundüberlegung der Kritik HomANNS ist dabei, dass viele der klassischen Ethiken, welche an das Gute im Menschen appellieren, noch zu sehr in einem alten Denken verhaftet seien, welches auf der Existenz einer traditionellen, relativ (wert)homogenen Gesellschaft beruhe, in welcher die Akteure häufig einander bekannt seien, so dass durch sozialen Druck eine Einhaltung von Normen erzwungen bzw. opportunistisches Verhalten begrenzt werden könne. Solch klassische Ethiken seien jedoch in modernen, durch Wettbewerb und Anonymität gekennzeichneten, funktional ausdifferenzierten Gesellschaften zunehmend unbrauchbar. ${ }^{242}$ Aus diesem Grund habe sich eine in der modernen Gesellschaft wirkmächtige „Wirtschafts- und Unternehmensethik [..] von Moralisieren $^{243}$, Appellieren ${ }^{244}$, Postulieren [...] tunlichst und peinlichst fernzuhalten.“245 Der Wettbewerb sei dabei grundsätzlich legitimierbar, wobei HOMANN auf das Argument von ADAM SMITH zurückgreift ${ }^{246}$, dass der Wettbewerb die beste Allokation von Ressourcen mit einem größten $\mathrm{Ma} ß$ an Wohlfahrt erzeuge. HOMANN rekurriert hierbei stark auf den empirisch gescheiterten Sozialismus, welcher sich als anreizschwache und letztlich unterlegene Mangelverwaltung erwiesen habe. ${ }^{247}$ Anstatt das wenige Vorhandene solidarisch zu teilen, sei es vielmehr geboten, mit ökonomischen Mitteln das Knappheitsproblem effizient zu lösen. HomanN verdeutlicht diese Situation häufig am Beispiel des ST. MARTIN: Anstelle der Teilung des Mantels aus Barmherzigkeit mit dem Bettler (traditionelles ethisches Gebot/Moralappell), welches nur dazu führe, dass nun beide nur einen halben Mantel besäßen, wäre es sinnvoller, ein Unternehmen zu gründen,

${ }^{242}$ Vgl. Homann, K. (2014), S. 45-48; Homann, K. (2002c), S. 182-183; Homann, K. (2002e), S. 49-50; Homann, K. (2002f), S. 6; Homann, K. (1995), S. 6-7; Homann, K. / Blome-Drees, F. (1992), S. 11-13 und S. 20-22.

${ }^{243}$ Vgl. zum Begriff des „Moralisierens“ auch die detaillierten Überlegungen bei MöhringHesse, M. (2013).

${ }^{244}$ HomANN spricht in diesem Zusammenhang sogar teilweise von „Appellitis“ (Homann, K. (1997), S. 34). Vgl. gleichermaßen auch Pies, I. (2015b), S. 2 sowie kritisch auch Gesang, B. (2016), S. 9. Den gleichen Begriff, wenngleich vor einem anderen konzeptionellen Hintergrund (vgl. hierzu auch Homann, K. (1994b), S. 122) gebraucht auch LuHMANN (vgl. Luhmann, N. (1993), S. 134).

${ }^{245}$ Homann, K. / Blome-Drees, F. (1992), S. 18-19.

${ }^{246}$ In diesem Kontext findet sich häufig die Referenz auf die vielzitierte Aussage ADAM SMITHS: „It is not from the benevolence of the butcher, the brewer, or the baker, that we expect our dinner, but from their regard to their own interest. We address ourselves, not to their humanity but to their self-love, and never talk to them of our own necessities but of their advantages." (Smith, A. (2009), S. 20).

${ }^{247}$ Vgl. Homann, K. / Lütge, C. (2013), S. 4 und S. 18; Homann, K. (2002f), S. 3-4; Homann, K. / Blome-Drees, F. (1992), S. 19. 
welches durch wettbewerbsfähige Produktion von Mänteln diesen Mangel beseitige und zugleich den Bettler mit Arbeit versorge, so dass sich dieser den Mantel selbst kaufen könne. ${ }^{248}$ Aus diesen Überlegungen ergibt sich nach HOMANN die vielfach übersehene, originär ethische Qualität der Marktwirtschaft ${ }^{249}$, mit der Schlussfolgerung, dass „Wettbewerb [.] solidarischer als Teilen“ ${ }^{“ 250}$ sei.

Obschon die Marktwirtschaft aus Sicht HomanNs für die moderne Gesellschaft gegenüber allen anderen Wirtschaftssystemen als vorzugswürdig gelten kann, kommt es im Rahmen dieser Wirtschaftsordnung in Kombination mit zunehmender Komplexität ${ }^{251}$ regelmäßig zu Dilemmasituationen ${ }^{252}$, welche Ethikkonzeptionen, die Individuen wie auch Unternehmen zu besserem Handeln auffordern, zu wenig beachteten. ${ }^{253}$ Grundproblem ist in der Realität komplexer moderner Gesellschaften die Möglichkeit zu opportunistischen Entscheidungen, indem sich z. B. Individuen aber auch Kollektive, welche normativ deviant handeln, auf Kosten anderer regelmäßig besserstellen können. ${ }^{254}$ Diese Situation lässt sich im klassischen Gefangenendilemma modellieren, auf welches HOMANN und

\footnotetext{
248 Vgl. Homann, K. (2008), S. 7.

${ }^{249}$ Vgl. Homann, K. (2015), S. 36-37; Homann, K. / Lütge, C. (2013), S. 46-47; Homann, K. (1995), S. 11; Homann, K. (1994b), S. 113; Homann, K. / Blome-Drees, F. (1992), S. $47-$ 50; Homann, K. (1990), S. 40. Vgl. hierzu auch Lütge, C. (2014), S. $28-29$.

${ }^{250}$ Homann, K. / Blome-Drees, F. (1992), S. 16 und S. 26. Vgl. ähnlich auch Homann, K. (1991), S. 45. Diesen Überlegungen folgen auch Vertreter einer traditionellen Betriebswirtschaftslehre, so z. B. ALBACH: ,Die Ethik des Schaffens hat Vorrang vor der Ethik des Teilens." (Albach, H. (2008), S. 8, in der Quelle ebenfalls herv.). Vgl. auch Albach, H. (2005), S. 817.

${ }^{251}$ Vgl. zur konzeptionellen Einführung in den Terminus der Komplexität Lingnau, V. / Brenning, M. (2018), S. 146-147; Lingnau, V. / Brenning, M. (2015), S. 457; Gerling, P. G. (2007), S. 23-25 sowie grundlegend Dörner, D. (2002), S. 58-59; Dörner, D. (1983), S. 19-24.

${ }^{252}$ Vgl. hierzu auch detailliert die Überlegungen in Homann, K. (2002a).

${ }^{253}$ Konzeptionell können dabei nach HoMANN die Rentabilität und die Moralvorstellungen in einer Vierfelder-Matrix gegenübergestellt werden. Während das Zusammenfallen von rentablen und moralischen Handlungen als unproblematisch erachtet werden kann, ergibt sich die klassische Herausforderung für die Ethik im Falle des moralischen oder ökonomischen Konfliktfalls, welcher von klassischen Ethiken zu wenig Beachtung geschenkt werde. Vgl. hierzu konzeptionell Homann, K. (1994b), S. 116-120; Pies, I. / Blome-Drees, F. (1993), S. 753; Homann, K. / Blome-Drees, F. (1992), S. 132-135 sowie grundlegend auch Homann, K. / Lütge, C. (2013), S. 78-79.

${ }^{254}$ Vgl. Homann, K. / Lütge, C. (2013), S. 17-18.
} 
seine Schüler häufig rekurrieren. ${ }^{255}$ Kernproblem ist im Rahmen dieses Dilemmas, dass es für jeden Akteur individuell betrachtet stets vorteilhaftig erscheint, opportunistisch zu handeln. Handeln jedoch beide Akteure in diesem Sinne, so führt dies zu einem insgesamt inferioren Zustand für beide Akteure. Diesen Zusammenhang verdeutlicht nochmals die nachfolgende Abbildung 3.8.

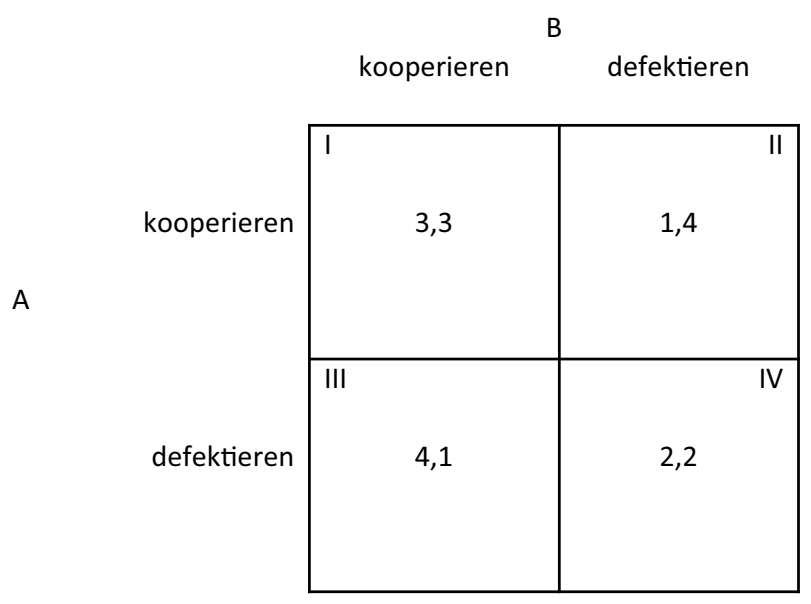

Abbildung 3.8 Auszahlungsmatrix des Gefangenendilemmas ${ }^{256}$

Eine solche unternehmerische konkurrenzinduzierte "Selbstschädigung“ ist dabei durchaus von der Wettbewerbsordnung intendiert, da sie für die Konsumenten zu niedrigeren Preisen ${ }^{257}$ führt. ${ }^{258}$ Eine durch die Unvollkommenheit des Marktes entstehende, fehlende Einpreisung externer Kosten (z. B. durch Umweltschädigungen) führt jedoch zu dem Problem, dass ein idealistisch motiviertes

${ }^{255}$ Vgl. Lütge, C. / Uhl, M. (2017), S. 91-94; Suchanek, A. (2015b), S. 59-63; Homann, K. (2014), S. 72-75; Homann, K. / Lütge, C. (2013), S. 24-27; Suchanek, A. (2007), S. 53-57; Homann, K. / Suchanek, A. (2005), S. 35-38; Homann, K. (2002a), S. 95-97; Homann, K. / Blome-Drees, F. (1992), S. 29-32; Homann, K. (1991), S. 32-33.

${ }^{256}$ Homann, K. / Lütge, C. (2013), S. 26.

${ }^{257}$ Eine Kollusion von Unternehmen (Kartellbildung) ist aus diesem Grunde auch wettbewerbsrechtlich verboten. Vgl. z. B. § 1 GWB (2021) sowie unionsrechtlich auch Art. 101, 102 AEUV (2012).

${ }^{258}$ Vgl. Homann, K. / Lütge, C. (2013), S. 25-31; Homann, K. (1994b), S. 110-111; Homann, K. / Blome-Drees, F. (1992), S. 29-35. 
Abweichen eines einzelnen Unternehmens im Preiswettbewerb ruinöse Konsequenzen für dieses nach sich ziehen kann $^{259}$, wenn sich die anderen Akteure durch weiterhin minimales Umwelt- oder Sozialengagement einen Kosten- und damit auch potentiellen Preisvorteil am Markt verschaffen können. ${ }^{260}$

Moralisieren, d. h. der Appell, solches Handeln zu unterlassen, sei in diesem Kontext überaus unfruchtbar, da dieser zum einen den Wettbewerbsdruck verkenne, welchem sich Unternehmen innerhalb einer Marktwirtschaft ausgesetzt sähen, zum anderen sei jedoch auch der reine Appell ${ }^{261}$, sich dauerhaft schlechterstellen $\mathrm{zu}$ müssen, um moralisch vorzugswürdig $\mathrm{zu}$ handeln, selbst ethisch überaus fragwürdig. HOMANN betont hierbei den alten Rechtsgrundsatz ${ }^{262}$, dass „Sollen“ immer auch „Können“ voraussetzt ${ }^{263}$, welches er auf die genannte Dilemmasituation im marktwirtschaftlichen Wettbewerb überträgt. So erscheine die Forderung, sich selbst im Vergleich zu anderen Akteuren einseitig dauerhaft schlechterstellen zu müssen (z. B. durch freiwilligen Gewinnverzicht

${ }^{259}$ In diesem Zusammenhang wird die ,unsichtbare Hand“ von SMITH folglich zur ,unsichtbaren Faust“ im Sinne des Bevölkerungsgesetzes nach MALTHus (vgl. hierzu Malthus, T. R. (2004), S. 13), da hier die Eigennutzverfolgung zu gesellschaftlich unerwünschten Zuständen (z. B. die Zerstörung der natürlichen Lebensgrundlagen) führt. Vgl. hierzu auch die Überlegungen in Stengel, O. (2016), S. 7; Lingnau, V. (2011a), S. 38; Pies, I. / Sardison, M. (2005), S. 13-14 sowie die Diskussion in Abschnitt 2.7.

${ }^{260}$ Vgl. Homann, K. (2014), S. 50-51; Homann, K. / Blome-Drees, F. (1992), S. 34. Dies gilt natürlich in einem strengen Sinne nur für vollkommene Märkte und entsprechende „Grenzanbieter“, welche keine Möglichkeit zur Differenzierung (z. B. durch nachhaltigere Produktion) besitzen. Vgl. hierzu die kritischen Ausführungen bei Steinmann, H. (2004), S. 111; Steinmann, H. / Löhr, A. (1994a), S. 108; Steinmann, H. / Oppenrieder, B. (1985), S. 176. Vgl. ähnlich auch Koslowski, P. (1998), S. 51-53.

${ }^{261}$ Homann und BLOME-DREES betonen in diesem Zusammenhang, dass ethische Appelle nicht per se ,überflüssig [seien], im Gegenteil, aber sie bedürfen der institutionellen Unterstützung.“ (Homann, K. / Blome-Drees, F. (1992), S. 28).

${ }^{262}$ Dieser lautet: „Ultra posse nemo obligatur“. Der Grundsatz nimmt auch in zahlreichen weiteren Beiträgen zur Ethik eine besondere Bedeutung ein. In diesem Sinne betont auch ENDERLE: „,Sollen“ impliziert bekanntlich ,Können““ (Enderle, G. (1991), S. 150). Ebenso betont auch HolzMANN: „Die Ethik muss also immer berücksichtigen, dass ein Sollen immer auch ein Können voraussetzt.“ (Holzmann, R. (2019), S. 5). Auch STEINMANN teilt prinzipiell diese Überzeugung und betont: „Sollen impliziert Können!“ (Steinmann, H. (2008a), S. 348). Vgl. ähnlich auch Göbel, E. (2017), S. 92; Gerum, E. / Mölls, S. (2009), S. 304; Hepfer, K. (2008), S. 34-35; Steinmann, H. (2004), S. 109; Lenk, H. / Maring, M. (1996), S. 6.

${ }^{263}$ Vgl. Homann, K. (2014), S. 250-252; Homann, K. / Lütge, C. (2013), S. 42; Homann, K. (2008), S. 9; Homann, K. (2002d), S. 257. 
bei SteINMANN und LOHR) geradezu illegitim ${ }^{264}$ und führe gar die Gefahr der Erosion von moralischen Standards mit sich (,der Ehrliche ist immer der Dumme $\left.^{\text {“265 }}\right)^{266}$, wodurch das eigentliche Ziel des Ethikers konterkariert werde: „Wenn die durch bestimmte institutionelle Arrangements gesetzten Sanktionsund Belohnungssysteme von der Art sind, daß moralisches Verhalten - nicht im Einzelfall, sondern - systematisch und dauerhaft mit ökonomischen Nachteilen ,bestraft ' wird, dann wird die Moral in einer komplexen, durch tiefe Arbeitsteilung und anonyme Austauschbeziehungen gekennzeichneten Welt sehr schnell erodieren; sie kann keinen Bestand haben." ${ }^{267}$ Traditionelle Ethiken, welche moralischeres Handeln nur einforderten, allerdings nicht die potentiell negativen Konsequenzen (z. B. nicht durch zunehmende Zahlungsbereitschaft der Kunden zu deckende Zusatzkosten) für die Handelnden und die Möglichkeit der Ausnutzung durch andere beachteten, drohen diese nach HoMANN potentiell zu überfordern, was sogar eigentlich wohlbegründete Anliegen unterminiere. ${ }^{268}$

Um wirtschaftsethische Probleme zu lösen, seien deshalb statt moralischer Appelle, anreizkompatible Strukturen zu entwickeln, so dass sich moralisches Handeln „rechne“ bzw. zumindest nicht bestraft werde. ${ }^{269}$ Um dies zu erzielen,

${ }^{264}$ In diesem Kontext postulieren HoMANN und LUTGE: „Keine Ethik [...] kann vom Einzelnen verlangen, dass er dauerhaft und systematisch gegen seine Interessen verstößt.“ (Homann, K. / Lütge, C. (2013), S. 43). Vgl. hierzu auch Homann, K. (2015), S. 34. Sein Schüler PIES formuliert noch schärfer: „Da jenseits des Könnens ein normatives Sollen nicht sinnvoll formuliert werden kann, verstoßen utopische Appelle in großer Regelmäßigkeit gegen den zutiefst moralischen Grundsatz ,ultra posse nemo obligatur'. Nicht zufällig sind deshalb Klagen über die Schlechtigkeit der Welt, insbesondere der Wirtschaftswelt, die stets mitlaufende Begleitmusik einer Appellitis, die sich darüber wundert und empört, dass ihre moralischen Appelle so wenig Befolgung finden." (Pies, I. (2015b), S. 2).

265 Vgl. ähnlich Homann, K. (2015), S. 30.

${ }^{266}$ Vgl. hierzu auch detailliert Homann, K. (2002f), S. 8-15, welcher vor der „Erosion der Moral durch Moralisieren“ warnt. Vgl. auch Homann, K. (2014), S. 64.

${ }^{267}$ Homann, K. / Blome-Drees, F. (1992), S. 94. In diesem Sinne betont HomanN: „Eine Gruppe, modelltheoretisch ein einzelner, kann eine moralische Institution zum Kippen bringen, weil sie bzw. er alle anderen zu ihrem eigenen Schutz vor größeren Nachteilen auf die Strategie der Defektion zwingen kann.“ (Homann, K. (1994b), S. 126). Vgl. zu dieser Situation der Asymmetrie auch Homann, K. (2015), S. 43; Homann, K. / Lütge, C. (2013), S. 27 und S. 40; Homann, K. / Suchanek, A. (2005), S. 36; Homann, K. (2002f), S. 14; Homann, K. (1990), S. 35.

${ }^{268}$ Vgl. Homann, K. (2015), S. 29-30; Homann, K. (2008), S. 4; Homann, K. (2002f), S. 14 18.

${ }^{269}$ Vgl. Homann, K. (2007), S. 5; Homann, K. (1997), S. 16-17. 
verschiebt HOMANN den Fokus der Ethik von der zentralen Verantwortungszuschreibung auf der Mikro- oder Mesoebene hin zur Makroebene der Wirtschaftsordnung. Die genannte ursprüngliche Dilemmasituation soll folglich dadurch aufgehoben werden, dass durch den Erlass sanktionsbewährter Gesetze für alle gleichermaßen erhöhte Standards gelten ${ }^{270}$, so dass keinem Akteur durch moralisches Handeln ein Nachteil mehr entstehe. Aus diesen Überlegungen kommt HOMANN zu der vielzitierten Schlussfolgerung: „Der systematische Ort der Moral in der Marktwirtschaft ist die Rahmenordnung. " 271 Dabei liegt der Fokus der Konzeption klar in der Frage, wie unter den Bedingungen der modernen Wirtschaft und Gesellschaft Ethik wirksam implementiert werden könne. ${ }^{272}$

Diese ordoethische Konzeption verdeutlicht HOMANN zumeist mit der Differenzierung von Spielregeln und Spielzügen. So fänden, analog zum Fußballspiel, die einzelnen Handlungen (Spielzüge) stets innerhalb von gegebenen Spielregeln statt. Anstelle nun auf die einseitige Änderung der Spielzüge durch Moralappelle zu setzen, fordert HOMANN die anreizkompatible Änderung der Spielregeln, so dass für alle Teilnehmer gleichermaßen veränderte Regeln gälten, wodurch es sich wieder lohne, moralisch zu handeln. ${ }^{273}$ Innerhalb dieser Spielregeln sei dann sämtliches Handeln legitim ${ }^{274}$ und nicht mehr ethisch kritisierbar: „Im Wettbewerb agieren die Unternehmen auf der Ebene der Spielzüge, die grundsätzlich

${ }^{270}$ Vgl. Homann, K. (2015), S. 35; Homann, K. / Lütge, C. (2013), S. 37.

${ }^{271}$ Homann, K. / Blome-Drees, F. (1992), S. 35, in der Quelle ebenfalls herv. Vgl. auch Homann, K. / Lütge, C. (2013), S. 77; Homann, K. / Pies, I. (2012), S. 68; Homann, K. (2002f), S. 7; Homann, K. (1995), S. 11; Homann, K. (1994b), S. 112; Homann, K. (1990), S. 38. Dies bedeutet zwar nicht, dass es keine weiteren Orte der Moral in der Marktwirtschaft gebe (vgl. z. B. Homann, K. / Blome-Drees, F. (1992), S. 37), dennoch wird dem Ansatz einer ethisch begründeten Modifikation der Rahmenordnung ein klares Primat zugesprochen. Vgl. hierzu auch Homann, K. / Blome-Drees, F. (1992), S. 114. Vgl. ähnlich auch die Überlegungen bei Homann, K. (2015): „Systematisch grundlegend ist die Rahmenordnung: Sie ist es, die moralisches Handeln erst ermöglicht.“ (S. 36).

${ }^{272}$ Vgl. Homann, K. / Lütge, C. (2013), S. 13; Homann, K. / Blome-Drees, F. (1992), S. 91. HomANN betont in diesem Zusammenhang häufig, dass zur Verhaltensänderung nicht der Fokus auf die Änderung individueller Handlungspräferenzen, sondern vielmehr auf die Änderung der empirischen Handlungsrestriktionen gelegt werden sollte. Vgl. Homann, K. I Blome-Drees, F. (1992), S. 103.

273 Vgl. Homann, K. (2015), S. 32 und S. 35-36; Homann, K. (2014), S. 62-63; Homann, K. / Lütge, C. (2013), S. 19-20; Homann, K. (2008), S. 14; Homann, K. / Suchanek, A. (2005), S. 40-43; Homann, K. (1994b), S. 111-112; Homann, K. / Blome-Drees, F. (1992), S. 20-29. ${ }^{274}$ In diesem Sinne betonen HOMANN und BLOME-DREES: „Das systemkonforme unternehmerische Gewinnstreben wird der Idee nach [...] durch die Rahmenordnung so kanalisiert, daß es als moralisch legitim angesehen werden kann." (Homann, K. / Blome-Drees, F. (1992), S. 114). 
moralfrei, jedoch nicht unmoralisch sind“275, wodurch es zu einer institutionell begründeten, moralischen Entlastung komme. Dabei folgt die Konzeption „dem Grundsatz, dass keine Ethik vom Einzelnen verlangen kann, dass er dauerhaft und systematisch gegen seine Interessen verstößt.“276

HOMANN fundiert damit seine Konzeption folglich erkennbar mit ökonomischen Überlegungen. Aufbauend auf der Existenz von Wettbewerb und dadurch induzierten Dilemmasituationen intendiert er, seine Ethikkonzeption zur Funktionslogik moderner Ökonomien kompatibel zu gestalten. ${ }^{277}$ Die Grundüberlegung, Ethik nicht gegen die Ökonomie (wie z. B. STEINMANN und LÖHR vorgeworfen), sondern mit den Mitteln der Ökonomie durchzusetzen, stellt damit das Leitparadigma der Konzeption HomanNS dar: „Normative Forderungen und Ideale lassen sich nicht gegen sondern nur durch die moderne Wirtschaft und Gesellschaft geltend machen.“278 In diesem Sinne betrachtet HomanNs Konzeption „,die Ökonomik nicht als Gegensatz zur Ethik, sondern als Fortsetzung der Ethik, aus der sie entstanden ist, mit anderen, besseren Mitteln.“279

Aus diesen Ausführungen wird der starke Fokus auf die Nutzung der Marktkräfte zur Lösung ethischer Fragestellungen bei HoMANN deutlich, welches auch die Bezeichnung als „Ethik mit ökonomischer Methode“ ${ }^{\prime 280}$ verdeutlicht. Als Testkriterium kommt hierbei, wie in der traditionellen Ökonomik üblich, der Homo oeconomicus $^{281}$ zum Einsatz, welcher, wie HOMANN und seine Schüler häufig

275 Homann, K. / Blome-Drees, F. (1992), S. 114.

${ }^{276}$ Homann, K. / Lütge, C. (2013), S. 16, in der Quelle ebenfalls herv.

${ }^{277}$ HomANn begründet seine Argumentation damit erkennbarerweise auf den klassischen Überlegungen der ordoliberalen Ö̈konomik. Vgl. hierzu z. B. paradigmatisch Homann, K. / Pies, I. (2012) sowie die klassischen Überlegungen bei Böhm, F. (1980); Eucken, W. (1975).

${ }^{278}$ Homann, K. / Blome-Drees, F. (1992), S. 111, in der Quelle ebenfalls herv. Vgl. ähnlich auch Homann, K. (2015), S. 24; Homann, K. (2002f), S. 3; Homann, K. (1994b), S. 110.

${ }^{279}$ Homann, K. / Lütge, C. (2013), S. 15. Vgl. hierzu auch Homann, K. (2015), S. 41; Homann, K. (2002b), S. 49; Homann, K. (2002d), S. 250.

${ }^{280}$ Homann, K. / Lütge, C. (2013), S. 13, in der Quelle ebenfalls herv. Vgl. ähnlich auch Homann, K. (2015), S. 23. Die von HomanN begründete, konzeptionelle Richtung der Wirtschaftsethik wird teilweise, gerade auch bei seinen Schülern, als „ökonomische Ethik“ bezeichnet (z. B. bei Suchanek, A. (2007); Pies, I. (2005)). HOMANN steht dieser Bezeichnung selbst jedoch kritisch gegenüber (vgl. hierzu Homann, K. (2015), S. 25).

${ }^{281}$ Unter dem Homo oeconomicus wird in der Regel ein vollständig opportunistischer Agent, welcher alle Handlungen nur zur Maximierung des eigenen Nutzens (i. d. R. in Zahlungsströmen ausgedrückt, vgl. Lingnau, V. (2011a), S. 36) evaluiert und folglich anderen Menschen gleichgültig gegenübersteht, sofern diese nicht instrumentell zur Maximierung des Eigennutzes dienlich erscheinen. Vgl. hierzu auch grundlegend die Ausführungen bei Kirchgässner, G. (2013), S. 13-15 und S. 47-48. Der Homo oeconomicus wird dabei vielfach als das 
betonen, in diesem Ansatz jedoch keinesfalls als reales Menschenbild aufgefasst werden sollte, sondern lediglich als Prüfkriterium opportunismusfester Spielregeln bzw. Institutionen diene: „Der homo oeconomicus stellt daher die Sonde dar, mit deren Hilfe der Ökonom die dauerhafte Funktionsfähigkeit von Institutionen angesichts der immer wieder auftretenden Defektionen untersucht. “282

Zusammenfassend kann damit festgehalten werden, dass HOMANN wirtschaftsethische Problemstellungen über eine makroethische Änderung der wirtschaftlichen Regeln zu lösen intendiert, wobei er besonders die Ausbeutungsgefahr moralisch Handelnder in den anonymen Strukturen der Wirtschaftssysteme der Moderne betont. ${ }^{283}$ Der Fokus der Wirtschaftsethik richtet sich in der Konzeption HomanNS folglich primär auf die Wirksamkeit ethischer Überlegungen in der Wirtschaftsrahmenordnung, wobei unternehmensethischen Fragestellungen nur ein subsidiärer Status im Falle von Regulierungsdefiziten oder unvollständiger Verträge zuerkannt wird $^{284}$, welches sich auch in der Regel in den recht knappen bzw. makroethisch orientierten Ausführungen zu diesem Themenfeld widerspiegelt. ${ }^{285}$

\subsubsection{Konzeptionelle Weiterentwicklung durch Suchanek und Pies}

Die wirtschaftsethische Schule HomanNs hat eine beachtliche Anzahl an Schülern hervorgebracht, welche auf seiner Konzeption aufbauen und teilweise auch eigenständige Weiterentwicklungen des Ansatzes vorangetrieben haben.

Standardmenschenbild der traditionellen, neoklassisch geprägten Ökonomik aufgefasst. Vgl. Kapeller, J. (2008), S. 1. Vgl. umfassend auch den Überblick bei Kühn, C. (2012), S. 113120. Zum historischen Hintergrund des Modells siehe die Ausführungen bei Suchanek, A. / Kerscher, K.-J. (2007), S. 259-263; Persky, J. (1995).

${ }^{282}$ Homann, K. / Blome-Drees, F. (1992), S. 95. Vgl. hierzu auch die Ausführungen in Lütge, C. / Uhl, M. (2017), S. 81-82; Homann, K. (2016), S. 47; Homann, K. (2015), S. 43; Homann, K. (2014), S. 101-105; Homann, K. / Lütge, C. (2013), S. 65-66; Suchanek, A. (2007), S. 179184; Suchanek, A. / Kerscher, K.-J. (2007), S. 253; Homann, K. / Suchanek, A. (2005), S. 418 426; Homann, K. (2003), S. 6; Homann, K. (1997), S. 17-22; Homann, K. (1995), S. 12-13; Homann, K. (1994a), S. 396-401 und S. 405-406; Homann, K. (1994b), S. 125-127.

283 Dies subsumiert HOMANN nochmals wie folgt: „Solange keine systematisch auf das zentrale Problem der Ausbeutbarkeit moralischer Vor- und Mehrleistungen zugeschnittenen Gegenmaßnahmen getroffen sind, sieht sich moralisches Handeln des Einzelnen im Wettbewerb mit ganz besonderen Schwierigkeiten konfrontiert und ist häufig gar nicht möglich.“ (Homann, K. (2014), S. 54).

${ }^{284}$ So dient die Unternehmensethik im Sinne HoMANNS lediglich zur Kompensation von Regulierungslücken, welche durch die Unvollkommenheit der Rahmenordnung (vgl. hierzu Homann, K. / Blome-Drees, F. (1992), S. 114-118) oder durch unvollständige Verträge (vgl. hierzu Homann, K. / Lütge, C. (2013), S. 75-77) entstehen.

${ }^{285}$ Vgl. z. B. die Überlegungen in Homann, K. (2015); Homann, K. / Lütge, C. (2013); Homann, K. (1995); Homann, K. / Blome-Drees, F. (1992). 
Im Weiteren sollen dabei besonders die zentralen Überlegungen zweier Schüler ${ }^{286}$, ANDREAS SUCHANEK sowie INGO PIES, vorgestellt werden, welche die Konzeption HoMANNS um einige Aspekte erweitert haben.

Betrachtet man in einem ersten Schritt das Werk SUCHANEKS, lässt sich feststellen, dass dieser den grundlegenden Überlegungen HoMANNS klar folgt bzw. diese konzeptionell voraussetzt. So betont auch SUCHANEK, dass wirtschaftsethische Problemstellungen in modernen Gesellschaften, welche nicht mehr durch einen weitgehend (z. B. religiös fundierten) Wertekonsens, sondern durch das „Faktum des Pluralismus “287 sowie durch in vielen Bereichen institutionalisierten Wettbewerb geprägt seien, nicht mehr appellativ, sondern vor allem institutionell durch Modifikation der (wirtschaftlichen) Rahmenordnung zu lösen seien. ${ }^{288}$ In diesem Sinne muss im konzeptionellen Verständnis SuchaneKs Moral in modernen Gesellschaften stets ,anreizkompatibel, $d$. $h$. vereinbar mit dem (wohlverstandenen bzw. reflektierten) Eigeninteresse sein.“289 Dabei folgt SUCHANEK im Wesentlichen der klassischen Differenzierung Homanns in Spielzüge und Spielregeln, wobei er diese jedoch noch um den dritten Aspekt eines gemeinsamen Spielverständnisses erweitert. So sei neben der Gestaltung anreizkompatibler Spielregeln auch bedeutsam, dass alle Spieler die Regeln verstünden bzw. stets klar sei, in welchem „Spiel“ sich die Akteure gerade befinden, d. h. welches Regelset gerade Gültigkeit beanspruche. ${ }^{290}$

${ }^{286}$ Darüber hinaus sind auch LUTGE und LIN-HI als bekannte Schüler HOMANNS zu nennen. Dabei fokussiert LIN-Hi jedoch relativ stark auf das Themengebiet der Corporate Social Responsibility und entwickelt dadurch die Konzeption HoMANNS nur bedingt weiter (vgl. z. B. Lin-Hi, N. / Suchanek, A. (2011); Lin-Hi, N. (2009)), während LƯTGE HomanNS Wirtschaftsethik programmatisch ohne größere Modifikation weiterführt (vgl. z. B. Lütge, C. / Uhl, M. (2017); Lütge, C. (2014); Lütge, C. (2012); Lütge, C. (2005)). Daher sei auf eine detailliertere Diskussion der Arbeiten der beiden Autoren in diesem Kontext verzichtet. ${ }^{287}$ Suchanek, A. (2007), S. 22, in der Quelle fett herv. Die Formulierung beruht auf den Überlegungen von RAWLS, welcher diese Entwicklung als „fact of pluralism“ (Rawls, J. (1987), S. 1) bezeichnet.

${ }^{288}$ Vgl. Suchanek, A. (2007), S. 20-27 sowie S. 92-95. Hierbei stellt SuCHANEK die moderne Gesellschaft den menschheitsgeschichtlich lange Zeit dominierenden, traditionell kleineren Gruppen gegenüber. Vgl. auch Suchanek, A. (2000), S. 12-16.

${ }^{289}$ Suchanek, A. (2007), S. 49, in der Quelle fett herv.

${ }^{290}$ Vgl. Suchanek, A. (2015a), S. 54-55; Suchanek, A. (2015b), S. 17-18 und S. 39-41; LinHi, N. / Suchanek, A. (2011), S. 65 und S. 75-76. 
Zweitens betont auch SUCHANEK die potentielle Differenz von Können und Sollen, welche es in wirtschaftsethischen Reflexionen stets zu bedenken gelte. Die grundlegenden Überlegungen HoMANNS werden auch hier von SUCHANEK konzeptionell erweitert, indem dieser das mögliche Auseinanderfallen von idealen Moralvorstellungen und dem empirisch Leistbaren mittels einer empirisch/normativen Adaption des klassischen Syllogismus nach ARISTOTELES ${ }^{291}$ analysiert. In diesem Sinne finden sich in einem solchen ,praktischen Syllogismus“292 im Obersatz die hypothetisch/idealfundierten Leitsätze, welche prinzipiell zu befolgen wären, während im Untersatz die empirischen Restriktionen eingeführt werden, die im Sinne eines Filters die Adaption dieser grundlegenden Regeln auf die konkrete Situation darstellen. Dem moralischen Ideal werden folglich die empirischen Handlungsrestriktionen gegenübergestellt. Durch dieses Verfahren erhält man nach SUCHANEK somit im dritten Schritt, analog zur klassisch-logischen Schlussfolgerung, das tatsächlich für das Individuum oder Kollektiv sinnvollerweise verpflichtende „Sollen“. ${ }^{293}$ Folge man diesen Überlegungen nicht, wie dies gerade viele klassisch-appellierende Ethiken regelmäßig aufzeigten, so begehe man einen ,normativistischen Kurz- bzw. Fehlschluss“, indem von dem moralischen Ideal direkt auf das „Sollen“ geschlossen werde. ${ }^{294}$ Diese Überlegungen fasst nochmals die nachfolgende Abbildung 3.9 zusammen.

Neben diesen grundlegenden Erweiterungen befasst sich SUCHANEK auch deutlich stärker mit den mesoethischen Überlegungen zur Begründung einer Unternehmensethik. Während, wie im vorigen Kapitel bereits diskutiert, im Rahmen der klassischen Konzeption HoMANNS das Themengebiet der Unternehmensethik nur relativ am Rande thematisiert wurde, stellt dies SUCHANEK wesentlich stärker in den Fokus der Betrachtung. Zentrale Überlegung SUCHANEKS ist es hierbei, Unternehmensethik in der Praxis als „Investition“ zu verstehen. ${ }^{295}$ So

\footnotetext{
${ }^{291}$ Vgl. hierzu nochmals die Überlegungen bei Aristoteles (2004), 100a 25-27 sowie grundlegend auch Aristoteles (2007).

292 Die Bezeichnung stammt von SuCHANEK. Vgl. hierzu die Ausführungen in Suchanek, A. (2015a); Suchanek, A. (2015b).

${ }^{293}$ Vgl. Suchanek, A. (2015b), S. 42-46; Suchanek, A. (2008b), S. 28; Suchanek, A. (2007), S. 30-31, S. 41 und S. 168; Suchanek, A. (2003), S. 4; Suchanek, A. / Waldkirch, R. (2002), S. 12; Suchanek, A. (2000), S. 32-33. Diese Überlegungen hat auch HOMANN mittlerweile übernommen. Vgl. hierzu Homann, K. (2014), S. 14.

${ }^{294}$ Vgl. Suchanek, A. (2015b), S. 183-184; Suchanek, A. (2007), S. 31; Suchanek, A. (2000), S. 35.

295 Vgl. Suchanek, A. (2015a), S. 51; Suchanek, A. (2015b), S. 12; Suchanek, A. (2015c), S. 60 und S. 69; Suchanek, A. (2008a), S. 3; Suchanek, A. (2007), S. 7.
} 
Abbildung 3.9 Der

normativistische

Fehlschluss nach

SUCHANEK $^{296}$
(1) Moralische Ideale

(2) Empirische Bedingungen

(3) Urteile, Forderungen

\section{Normativistischer Fehlschluss:}

Vernachlässigung relevanter empirischer

Bedingungen bei der Herleitung ethischer

Urteile

verzichte das Unternehmen, welches dabei als handlungsfähige Einheit bzw. korporativer Akteur verstanden wird ${ }^{297}$, heute auf Handlungsoptionen, um damit zukünftige Vorteile zu erzielen. Dies sei notwendig, da reales wirtschaftliches Handeln stets unter mehr oder minder großer Unsicherheit stattfinde. In diesem Sinne könne z. B. der Verzicht auf die Nutzung aller diskretionären Handlungsspielräume $^{298}$ in der Gegenwart zum Aufbau von Vertrauen führen, welches sich in der Zukunft für das Unternehmen, aber auch für alle weiteren Betroffenen auszahle. ${ }^{299}$ Es geht folglich darum, ,auf kurzfristig attraktive Handlungsalternativen zu verzichten, sich also in diesem Sinne selbst zu binden."300 In diesem Kontext hebt SUCHANEK hervor: „Die vielleicht präziseste Möglichkeit, diesen

\footnotetext{
296 In Anlehnung an Suchanek, A. (2015b), S. 184.

${ }^{297}$ Vgl. Suchanek, A. (2015b), S. 255; Suchanek, A. (2007), S. 117-119; Suchanek, A. (2003), S. 12.

${ }^{298}$ Dies gilt, wie SUCHANEK hervorhebt, insbesondere vor dem Hintergrund der heutigen globalisierten Wirtschaftswelt. Vgl. Suchanek, A. (2015c), S. 61.

${ }^{299}$ In diesem Kontext betont SUCHANEK: ,(Corporate) Integrity can be seen as an asset in that a notably upright corporation can more easily find cooperation partners (i.e. customers, suppliers, employees, investors, etc.), and in addition they can expect that decisions and negotiations will be reached relatively quicker and in a less complicated way, which makes the entire process more cost-efficient." (Suchanek, A. (2008a), S. 7). Ähnlich argumentiert auch WIELAND, welcher innerhalb seiner Konzeption die Bedeutung von Unternehmensethik mit einer Senkung von Transaktionskosten begründet. Vgl. hierzu die Ausführungen in Abschnitt 3.4.5.1.

${ }^{300}$ Suchanek, A. (2015b), S. 47, in der Quelle fett herv.
} 
Zusammenhang zu beschreiben, bietet der Begriff der Investition. Jede Investition zielt darauf ab, durch heutigen Verzicht auf Konsum, worin immer dieser bestehen mag, sich künftig besserzustellen.“" ${ }^{301}$ Diese Überlegungen fokussieren demnach auf das Erreichen eines PARETO-superioren Zustandes, womit explizit davon ausgegangen wird, dass (zumindest langfristig) alle Beteiligten durch die unternehmensethisch begründete „Investition“ bessergestellt werden: „Es geht um die eigene und zugleich allgemeine Besserstellung. "302 SUCHANEK verknüpft diese unternehmensethischen Überlegungen mit der klassischen Goldenen Regel der Reziprozität, welche als normative Grundkonstante in der menschlichen Entwicklung gilt. ${ }^{303}$ Hieraus ergibt sich subsumierend nach SUCHANEK schließlich die ,ökonomisch reformulierte und erweiterte Goldene Regel: Investiere in die Bedingungen der gesellschaftlichen Zusammenarbeit zum gegenseitigen Vorteil!"304

Als weiterer zentraler Schüler, welcher die wirtschaftsethische Konzeption HomAnNs weiterentwickelt hat, kann PIES mit seinem explizit als „ordonomische Ethik “305 bezeichneten Ansatz aufgefasst werden. Auch dieser beruht im Kern nach wie vor auf den zentralen Überlegungen von HoMANN, erweitert diese jedoch wieder um konzeptionelle Details. So stellt auch PIES fest, dass moderne Gesellschaften nur noch über einen Regelkonsens und nicht mehr primär über einen existierenden Wertekonsens gedacht werden könnten ${ }^{306}$, welches wie auch bei HOMANN Implikationen hinsichtlich der Gestaltung wirtschaftsethischer Maßnahmen aufweist. So folgt auch PIES der Aufforderung, nicht (im „,vormodernen“ Sinne) individuelles wie kollektives Handeln ${ }^{307}$ mittels Appell

${ }^{301}$ Suchanek, A. (2007), S. 50, in der Quelle fett herv.

302 Suchanek, A. (2007), S. 8, in der Quelle fett herv. Vgl. hierzu auch Suchanek, A. (2003), S. 14.

${ }^{303}$ Vgl. Suchanek, A. (2015b), S. 187.

${ }^{304}$ Suchanek, A. (2007), S. 39, in der Quelle fett herv. Vgl. ähnlich auch Suchanek, A. (2015a), S. 59; Suchanek, A. (2015b), S. 17; Suchanek, A. (2015c), S. 60; Suchanek, A. (2008a), S. 4; Suchanek, A. (2003), S. 9.

${ }^{305}$ Hierdurch wird auch klar, dass sich PIES wiederum vornehmlich mit ordo- bzw. rahmenordnungsethischen Fragestellungen befasst.

${ }^{306}$ Vgl. Pies, I. (2012b), S. IX-XII; Pies, I. (2003), S. 9-15. Inwiefern diese Position jedoch vollständig zu überzeugen vermag, kann mit guten Gründen bezweifelt werden. So weist auch SUCHANEK darauf hin, dass selbst bei der Annahme einer dominierenden Koordination durch Regeln, es immer noch spezifischer Grundwerte, wie z. B. der Toleranz, bedarf. Vgl. hierzu Suchanek, A. (2007), S. 22.

${ }^{307}$ In diesem Sinne betont PIES: ,[T] $]$ he ordonomic model allows actors to be individuals, groups of individuals, organizations, groups of organizations and even states or groups of states [...].“ (Pies, I. (2016b), S. 149). 
zur Änderung der Präferenzen zu beeinflussen, sondern die Rahmenbedingungen (anreizkompatibel) so zu gestalten, dass sich innerhalb dieses modifizierten Rahmens das individuelle oder kollektive Handeln entsprechend ändere. ${ }^{308}$ Die klassische Differenzierung zwischen Handlungen und institutionellen Rahmenbedingungen wird nun von ihm spieltheoretisch interpretiert und in ein dreiteilig erweitertes Mehrebenenschema gebracht ${ }^{309}$ : Die grundlegenden Interaktionen im Wirtschaftssystem werden dabei als Basisspiel rekonstruiert, dessen Regeln wiederum durch das übergeordnete „Metaspiel“ der Politik gestaltet werden. Darüber hinaus, und in Erweiterung der Überlegungen HoMANNS, führt PIES als dritte Ebene schließlich auch noch ein Meta-Metaspiel der Öffentlichkeit ein, welches wiederum die Rahmenbedingungen für das Metaspiel der Politik definiert. ${ }^{310}$

Mithilfe dieses konzeptionellen Rahmens wird nun die Interaktion auf und zwischen den Ebenen analysiert, wobei PIES für jede Stufe im Kontext der modernen Gesellschaftsstrukturen ein spezifisches Versagen diagnostiziert: Während im Basisspiel ein Marktversagen festgestellt wird, konstatiert PIES für das Metaspiel ein Politikversagen, welches sich u. a. durch immer noch dominant appellative Beeinflussungsversuche des Politiksystems gegenüber der Wirtschaft zeige. ${ }^{311}$ Darüber hinaus sei jedoch das Politiksystem selbst durch die Eigenlogik des kurzfristigen Wahlerfolges gekennzeichnet, welches als immanenter Systemzwang im öffentlichen Diskurs immer noch zu wenig Beachtung fände, weshalb PIES für das Meta-Metaspiel der Öffentlichkeit ein Diskursversagen feststellt. ${ }^{312}$ Dabei hänge das Versagen des öffentlichen Diskurses nicht unwesentlich mit durch traditionelle Moralvorstellungen induzierten Denkblockaden zusammen, welche sich wiederum auf der zweiten Stufe in regelmäßigen Handlungsblockaden bei der (Neu-)Gestaltung der wirtschaftlichen Rahmenordnung äußerten. ${ }^{313}$ Zur Lösung der aufgezeigten Versagensprobleme erweise sich der von ihm entwickelte ordonomische Ansatz als besonders fruchtbar. Dieser könne

${ }^{308}$ Vgl. Pies, I. (2015b), S. 1-2.

${ }^{309}$ Vgl. Pies, I. (2010), S. 258.

${ }^{310}$ Vgl. Pies, I. (2017), S. 12; Pies, I. (2016a), S. 26; Pies, I. (2016b), S. 149; Pies, I. (2015a), S. 82; Pies, I. (2015b), S. 13; Pies, I. (2012a), S. 230-235; Pies, I. (2012c), S. 25; Pies, I. (2010), S. 256-257; Pies, I. / Beckmann, M. / Hielscher, S. (2010), S. 268; Pies, I. / Hielscher, S. / Beckmann, M. (2009b), S. 386.

${ }^{311}$ In diesem Sinne kritisiert PIES prägnant die klassisch-individualethisch geprägten Konzeptionen und hebt hervor: „Die überdehnte Individualethik neigt zum überspannten Moralisieren." (Pies, I. (2010), S. 253).

312 Vgl. Pies, I. (2015a), S. 81-82.

313 Vgl. Pies, I. (2015a), S. 106; Pies, I. (2010), S. 258; Pies, I. (2005), S. 1; Pies, I. (2000), S. 315. Vgl. hierzu ähnlich auch: „Die Überdehnung der Individualethik behindert 
darin unterstützen, die genannten faktischen Problemfelder nicht nur konzeptionell zu durchdringen, sondern auch der Gesellschaft Lösungsmöglichkeiten hierzu zu offerieren. Dabei operiert das aufgezeigte Schema primär top-down, d. h. in einem ersten Schritt sei der öffentliche Diskurs so neuzugestalten, dass dieser keine Fehlanreize mehr für das untergeordnete politische System setze, welches dann wiederum die ,richtigen“ Anreize bzw. Rahmenbedingungen für das Wirtschaftsspiel bzw. die einzelnen Akteure zu implementieren vermag. ${ }^{314}$ Diese Überlegungen fasst nochmals die nachfolgende Abbildung 3.10 zusammen.

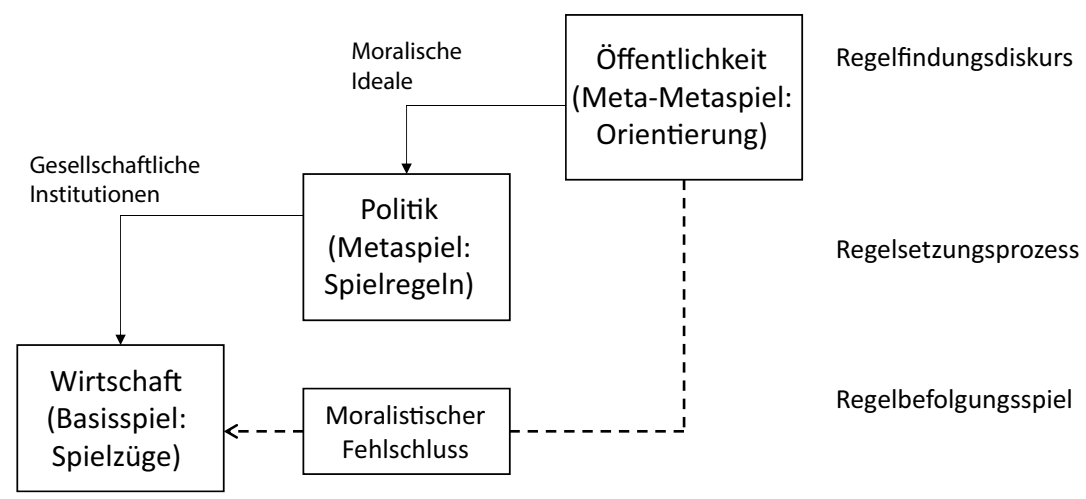

Abbildung 3.10 Das ordonomische Drei-Ebenen-Schema nach PIES ${ }^{315}$

Das im Kontext der Implementierung von Moral sowohl auf der gesellschaftlichen als auch politischen Ebene existierende, originäre Problem führt

konstruktiv(istisch)e Versuche, durch eine (Re-)Formierung des Ordnungsrahmens und seiner Anreizwirkungen moralische Anliegen nachhaltig zu verwirklichen." (Pies, I. (2015b), S. 14).

314 Vgl. Pies, I. (2015a), S. 93-94 und S. 106-107.

315 Pies, I. (2012c), S. 25. Hierbei diskutiert PIES auch die Problematik des ,moralistischen Fehlschlusses“, welcher in seiner Konzeption darauf hinweist, dass von der Öffentlichkeit in der Wirtschaft z. B. nachhaltigeres Handeln häufig appellativ eingefordert, dabei aber die Ebene der Rahmensetzung durch die Politik übersprungen und die Änderung der Wirtschaftspraxis auch ggf. durch Wahlentscheidungen nicht anreizkompatibel für die Politik unterstützt wird. Vgl. zu weiteren Anwendungen des Drei-Ebenen-Schemas auch Pies, I. (2016a), S. 26; Pies, I. (2015a), S. 82; Pies, I. (2015b), S. 13; Pies, I. / Beckmann, M. / Hielscher, S. (2011), S. 20; Pies, I. / Beckmann, M. / Hielscher, S. (2010), S. 268; Pies, I. / Hielscher, S. / Beckmann, M. (2009a), S. 326; Pies, I. / Hielscher, S. / Beckmann, M. (2009b), S. 386; Pies, I. / Hielscher, S. / Beckmann, M. (2009c), S. 193. 
PIES, ähnlich wie auch HOMANN und SUCHANEK, zentral auf ein vormodernes, vornehmlich appellativ operierendes sowie auf Mildtätigkeit und Umverteilung beruhendes intuitives Moralverständnis in der Bevölkerung, welches die ethische Qualität der Marktwirtschaft und die Vorzüge kapitalistischen Eigennutzstrebens noch nicht mit der moralischen Intuition in Deckung gebracht habe, zurück. In diesem Sinne passe die Semantik der Moral vielfach noch nicht zur gesellschaftlichen Realität bzw. Sozialstruktur der Moderne. Grundaufgabe der ordonomischen Ethik ,,als Theorie für die Praxis“316 sei daher auch die doppelte Reflexion der gesellschaftlichen Realitäten wie auch der eingesetzten Implementierungsvorschläge von Moral. Hieraus ergeben sich folglich zwei Aufgaben der ordonomischen Ethik: „Die Ordonomik fragt zum einen nach der Moraltauglichkeit unserer Gesellschaft und zum anderen nach der Gesellschaftstauglichkeit unserer Moral.“317 Sie ist damit ,ein Forschungsprogramm zur Analyse (des Zusammenhangs) von Sozialstruktur und Semantik."318 Die erstere Teilüberlegung dient dabei der kontinuierlich kritischen Reflexion der gesellschaftlichen Realitäten und inwiefern diese moralisch definierten Anforderungen genügen. Der zweite Aspekt fokussiert dann auf das Problem der fehlenden Übereinstimmung moralischer Intuitionen und den „Funktionalitätserfordernissen einer modernen (Welt-)Gesellschaft“319. Es geht in diesem Kontext folglich darum, „die Denkrahmen gesellschaftlicher Kommunikation - die kategorialen ,mindsets ‘, die zugrunde liegenden ,mental models“ oder, intellectual frames ${ }^{\text {‘ }}$ - zu untersuchen und im Bedarfsfall Anstöße für eine Weiterentwicklung anzuregen, so dass eine gemeinsame Problemwahrnehmung und mithin eine gesellschaftliche Verständigung möglich wird." 320

So würden im Rahmen der immer noch herrschenden Moralsemantik häufig unüberbrückbare Zielkonflikte, z. B. zwischen Moral und Gewinnstreben unterstellt, welche jedoch nicht notwendigerweise unauflösbar bleiben müssten. Grundidee der ordonomischen Ethik ist es damit, neben der Problemanalyse auch

\footnotetext{
316 Pies, I. (2015a), S. 79. Ähnlich betonen auch PIES, BECKMANN und HIELSCHER: „Das ordonomische Forschungsprogramm versteht sich nicht als l'art pour l'art, sondern als eine Theorie, die praktisch werden will." (Pies, I. / Beckmann, M. / Hielscher, S. (2009), S. 17). Vgl. auch Pies, I. / Hielscher, S. / Beckmann, M. (2009a), S. 330.

${ }^{317}$ Pies, I. (2015a), S. 79 sowie ähnlich Pies, I. (2016b), S. 150. Vgl. hierzu auch Pies, I. (2013), S. 1.

318 Pies, I. / Beckmann, M. / Hielscher, S. (2009), S. 6.

319 Pies, I. (2015a), S. 79.

${ }^{320}$ Pies, I. / Beckmann, M. / Hielscher, S. (2009), S. 6, in der Quelle ebenfalls herv.
} 
die scheinbaren Zielkonflikte mittels ordnungsethischer Maßnahmen zu überwin$\operatorname{den}^{321}$ und diese wenn möglich in eine PARETO-superiore ${ }^{322}$ (d. h. Win-Win-) Situation $^{323}$ zu transformieren, welches PIES auch als orthogonale Positionierung bezeichnet. ${ }^{324}$ In diesem Sinne geht es im Rahmen der Ordonomik um die Verdeutlichung der Möglichkeiten bzw. Erschließung der „Potenziale wechselseitiger Besserstellung “325. Es sei folglich darauf hinzuweisen, ,dass es nicht darum geht, die eine Interessengruppe zu Lasten der anderen Interessengruppe besserzustellen [...]. Stattdessen kommt es darauf an, eine Zone gemeinsamer Interessen zu identifizieren, in der sich eine wechselseitige Besserstellung ${ }^{326}$, eine Win-WinLösung, organisieren lässt [...]. “327 Dieser Zusammenhang wird nochmals in der nachfolgenden Abbildung 3.11 aufgezeigt.

Neben der detaillierteren konzeptionellen Ausarbeitung der grundlegenden makroethischen Überlegungen HOMANNS, hat sich auch PIES unternehmensethischen Fragen zugewandt. So erkennt PIES trotz der auch in seiner Konzeption zentralen ordoethischen Überlegungen an, dass die wirtschaftliche Rahmenordnung zum einen Regelungslücken aufweise und zum anderen die bisherige

${ }^{321}$ Dies steht ganz in der Tradition HomANNS, denn auch in diesem ,Ansatz geht es darum, zur Verwirklichung moralischer Anliegen beitragen zu helfen - mit Hilfe von institutionellen Arrangements, die unter Umständen völlig kontra-intuitiv funktionieren, so dass eine ethische Aufklärung moralischer (und insbesondere: moralisierender) Vorurteile nötig wird, um mehr Moral möglich zu machen und wirklich werden zu lassen.“ (Pies, I. (2010), S. 259).

${ }^{322}$ Vgl. Pies, I. / Hielscher, S. / Beckmann, M. (2009a), S. 322.

${ }^{323}$ Als klassisches, vielzitiertes Beispiel könnte hier z. B. die Verdoppelung des Arbeitsstundenlohnes auf $5 \$$ bei Ford im Jahre 1914 herangezogen werden, welche zwar eigentlich die Lohnkosten erhöhte, aber durch längere Mitarbeiterbindung und höhere Motivation insgesamt zu einer Produktionskostensenkung sowie zu einer erhöhten Kaufkraft führte, die es den Mitarbeitern wiederum ermöglichte, die eigenen Produkte zu kaufen. Vgl. hierzu Mankiw, N. G. (2015), S. 211; Kaufman, B. E. (2010), S. 95; Pies, I. / Hielscher, S. / Beckmann, M. (2009b), S. 391-392; Pies, I. (2007), S. 2.

${ }^{324}$ Vgl. Pies, I. (2017), S. 10; Pies, I. (2016a), S. 25; Pies, I. (2015a), S. 92; Pies, I. (2012c), S. 23; Pies, I. / Beckmann, M. / Hielscher, S. (2009), S. 17; Pies, I. / Hielscher, S. / Beckmann, M. (2009b), S. 380; Pies, I. (2007), S. 1. In diesem Sinne betonten PIES und BLOME-DrEeS bereits Mitte der 1990er Jahre: „Das erklärte Ziel ist eine Kompatibilität von Gewinn und Moral.“ (Pies, I. / Blome-Drees, F. (1995), S. 177).

325 Pies, I. (2015a), S. 82.

${ }^{326}$ Als ein an die klassischen Überlegungen bei Ford angelehntes Beispiel könnte hier z. B. die bessere Bezahlung von Angestellten in Niedriglohnländern herangezogen werden, welche diese dazu befähigen würde, verstärkt die eigenen Produkte zu kaufen, so dass hierdurch auch neue Absatzmärkte erschlossen werden könnten.

${ }^{327}$ Pies, I. (2015a), S. 92. 
Abbildung 3.11 Die orthogonale Positionierung nach PIES ${ }^{328}$

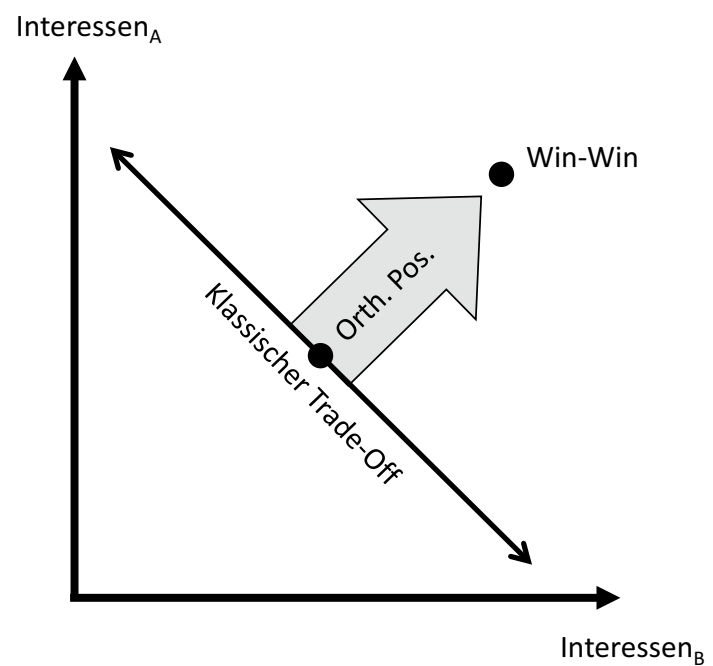

Wirtschaftsrahmenordnung zunehmend nicht mehr hinreichend als alleinig legitimierende Handlungsgrundlage im unternehmerischen Kontext herangezogen werden könne. Hierbei konstatiert PIES gerade im deutschen Kontext ${ }^{329}$ eine signifikante ,Akzeptanzkrise: Ein dramatischer Ansehensverlust betrifft (a) das System der Marktwirtschaft, (b) die Unternehmen als wichtige Akteure in diesem System, (c) die Manager als Führungskräfte in Unternehmen und (d) das Gewinnstreben als Organisationsprinzip der Unternehmen.“330

\footnotetext{
328 In Anlehnung an Pies, I. (2015a), S. 92. Vgl. ähnlich auch Pies, I. (2016a), S. 25; Pies, I. (2015b), S. 16; Pies, I. (2012c), S. 23; Pies, I. / Hielscher, S. / Beckmann, M. (2009b), S. 380; Pies, I. (2007), S. 1.

${ }^{329} \mathrm{Vgl}$. im internationalen Kontext ähnlich auch HORVÁTH und BARTON, welche hervorheben ,that public trust in capitalism has sunk to an all-time low in many developed countries [...].“ (Horváth, D. / Barton, D. (2016), S. 139).

${ }^{330}$ Pies, I. (2007), S. 1. In diesem Sinne betonen auch PIES, HIELSCHER und BECKMANN: „Empirische Untersuchungen zeigen [...], dass das Vertrauen der Bevölkerung in das System der Marktwirtschaft, in die korporativen Akteure der Marktwirtschaft, in deren Führungskräfte und in das handlungsleitende [d. h. ökonomische, d. V.] Prinzip der Unternehmen [...] auf breiter Front historische Tiefststände erreicht hat.“ (Pies, I. / Hielscher, S. / Beckmann, M. (2009a), S. 324).
} 
Aus diesen Gründen ergebe sich die Notwendigkeit originärer Unternehmensethik, welche PIES als Nutzbarmachung von Moral als Produktionsfaktor auffasst. ${ }^{331}$ Vergleiche man jedoch die gestiegenen Anforderungen zur Rechtfertigung wirtschaftlichen Handelns, mit welchen sich Führungskräfte in zunehmendem Maße konfrontiert sähen, mit deren faktischen Fertigkeiten, so zeige sich, dass reale Manager noch vielfach zu wenig ethische Grundlagenbildung besäßen, um in einem wohlbegründeten Diskurs mit den Stakeholdern bestehen zu können ${ }^{332}$, woraus PIES einen klaren Bildungsauftrag für die universitäre Lehre ableitet. In diesem Sinne sei insbesondere auch im wirtschaftswissenschaftlichen Studium ein stärkerer Fokus auf die didaktische Vermittlung von ethischen Grundlagen geboten, welches für die moderne Wirtschaft unentbehrliches Orientierungswissen liefere. ${ }^{333}$ So werde von Führungskräften zunehmend erwartet, ,dass sie ihre Position verständlich vertreten (können), etwa indem sie zu den Folgen ihres Handelns in systemischen Zusammenhängen überzeugend Rede und Antwort stehen. Dazu gehört die Fähigkeit, zu normativ strittigen Auseinandersetzungen konstruktiv Stellung zu beziehen. “334 Dabei sei eine fundierte diskursethische Ausbildung zentral, denn „,a company can engage in win-win

${ }^{331}$ Vgl. Pies, I. / Beckmann, M. / Hielscher, S. (2011), S. 17-18; Pies, I. (2010), S. 259; Pies, I. (2007), S. 2.

332 Ähnliche Überlegungen finden sich bereits in den 1980er Jahren bei STEINMANN und OPPENRIEDER, welche hervorhoben: „Für die Managementaus- und -weiterbildung entsteht neben der Vermittlung ökonomisch-technischer Fertigkeiten mit der Forderung nach einer Unternehmensethik die Aufgabe, die (zukünftigen) Führungskräfte in ethisch-normative Argumentationen (,praktische Urteilsfähigkeit") einzuüben." (Steinmann, H. / Oppenrieder, B. (1985), S. 179). SUCHANEK betont ebenso die Bedeutung einer verbesserten wirtschaftsethischen Managerbildung, wobei sich zwei zentrale Handlungsfelder ergäben: „On one hand the competence to organize the reconciliation of morality and profit by creating appropriate governance structures and on the other hand the ability to communicate authentically the reasons as well as the limits to do so." (Suchanek, A. (2008a), S. 9). Auch an anderer Stelle hebt SuCHANEK hervor: ,An diesem Punkt zeigt sich zugleich die Wichtigkeit der zweiten Fähigkeit, die mit Argumentationskompetenz beschrieben werden kann, der - bedauerlicherweise - im Rahmen betriebswirtschaftlicher Ausbildung kaum Bedeutung beigemessen wird: die Fähigkeit, als Entscheidungsträger in Unternehmen die eigenen Strategien und Entscheidungen nach innen und nach außen kommunizieren und begründen zu können, auch und gerade dann, wenn komplexe moralische Probleme involviert sind." (Suchanek, A. (2008b), S. 27, in der Quelle ebenfalls herv.).

${ }^{333}$ Vgl. detailliert Pies, I. / Beckmann, M. / Hielscher, S. (2010), S. 271-273; Pies, I. / Beckmann, M. / Hielscher, S. (2009), S. 17-19; Pies, I. / Hielscher, S. / Beckmann, M. (2009a), S. 324-329. Vgl. hierzu auch die Überlegungen bei Ulrich, P. (1994), S. 76.

334 Pies, I. / Beckmann, M. / Hielscher, S. (2009), S. 18-19. Vgl. ähnlich auch Pies, I. / Beckmann, M. / Hielscher, S. (2010), S. 272. 
interactions, and thus create value, only if it knows who its relevant stakeholders are and if it understands their interests, which, in a rapidly changing world, is a constant challenge. In order to be successful, the company needs to listen and then learn from what it hears - discourse is essential to this process. “335 In diesem Sinne gehe es letztlich im Rahmen der wirtschaftsethischen Bildung, wie PIES und seine Schüler HIELSCHER und BECKMANN vielfach betonen, gerade nicht darum, Studierende der Wirtschaftswissenschaften (primär) „zu besseren Menschen zu erziehen, sondern sie hat das Ziel, die Studierenden zu besseren (erfolgreicheren) Managern auszubilden.“336

\subsubsection{Die integrative Wirtschaftsethik ULRICHS}

Obschon die zwei aufgezeigten Hauptströmungen der Wirtschaftsethik eine breite Resonanz in der Öffentlichkeit erfahren sowie viele akademische Schüler hervorgebracht haben, so hat sich an beiden Positionen auch deutliche Kritik entwickelt. Einer der bekanntesten Kritiker beider Positionen ist PETER ULRICH, welcher mit seiner integrativen Wirtschaftsethik einen „dritte[n] Weg“337 zur Auflösung des Konflikts zwischen einer freiwilligen Gewinnverzichtslogik und einer dominant makroethischen Anreizsteuerung intendiert. So kritisiert ULRICH sowohl die Position STEINMANNS und LOHRS als auch die Konzeption nach HoMANN, da diese im Kern nicht das traditionelle ökonomische Paradigma überwinden bzw. dieses ungeprüft in ihren Konzeptionen übernehmen würden, weshalb er beiden Positionen einen systematischen „Reflexionsstopp“338 vorwirft. An der Position STEINMANNS und LÖHRS kritisiert ULRICH im Speziellen, dass diese von einer amoralischen bzw. moralfreien Wirtschaft ausgingen, welcher nun die Ethik lediglich als Korrektiv gegenübergestellt werde. Es handele sich in diesem Fall folglich nur um eine situative bzw. ,korrektive Wirtschaftsethik“339. Faktisch sei jedoch

\footnotetext{
335 Pies, I. / Beckmann, M. / Hielscher, S. (2010), S. 270.

336 Pies, I. / Hielscher, S. / Beckmann, M. (2009a), S. 324. Vgl. ähnlich auch Pies, I. / Beckmann, M. / Hielscher, S. (2011), S. 33; Pies, I. / Beckmann, M. / Hielscher, S. (2010), S. 267. Diese Überlegungen finden sich bei PIES und BLOME-DREES bereits Mitte der 1990er Jahre: „Unserem Verständnis nach bedeutet das freilich, sie nicht zu Philosophen, sondern zu besseren Ökonomen in dem Sinne auszubilden, daß sie die normativen Implikationen ihres Tuns reflektiert handhaben können [...].“ (Pies, I. / Blome-Drees, F. (1995), S. 177).

${ }^{337}$ Ulrich, P. (2017), S. 7; Ulrich, P. (1994), S. 78; Ulrich, P. (1989), S. 23, in den Quellen teilweise herv.

338 Ulrich, P. (2017), S. 14; Ulrich, P. (2016a), S. 15. Vgl. auch Ulrich, P. (2004b), S. 17.

${ }^{339}$ Ulrich, P. (2016a), S. 135; Ulrich, P. (2015a), S. 216, in den Quellen fett herv.
} 
z. B. die Entscheidung für eine Wettbewerbsordnung und deren Ausgestaltung stets eine normative Frage, welche durch die Ethik grundlegend reflektiert werden müsse, anstelle nur eine situative Verhaltensänderung (bei STEINMANN und LOHR: Gewinnverzicht) zu fordern. Ein solcher Ansatz bliebe daher, wie viele klassische Ethikkonzeptionen in einem Zwei-Welten-Konzept ${ }^{340}$ verhaftet, in welchem sich Ethik und Ökonomik unversöhnlich gegenüberstünden, welches jedoch gerade nach ULRICH zu überwinden sei. ${ }^{341}$

Aber auch die alternative Position HOMANNS wird von ULRICH überaus kritisch rezipiert. So intendiert HOMANN zwar in einem spezifischen Sinne die genannte Zwei-Welten-Auffassung zu überwinden, setze dabei jedoch nach ULRICH systematisch und methodisch falsch an, indem er die Ethik der ökonomischen Rationalität instrumentell unterzuordnen versuche und damit das ökonomische Prinzip in der Marktwirtschaft überhöhe. In dieser Auffassung diene Ethik lediglich als Hilfsmittel (,funktionalistische Wirtschaftsethik ${ }^{\text {‘342), }}$ um einer ökonomischen Rationalität noch bessere Geltung zu verschaffen. Scheinbar gegenwärtig unauflösbare ökonomische Sachzwänge seien jedoch wiederum nicht unkritisch zur Konzeption einer Wirtschaftsethik zu übernehmen. Gleiches gelte auch für die axiomatische Setzung einer wettbewerbsorientierten Wirtschaftsordnung, da auch diese wiederum letztlich eine normativ zu begründende Entscheidung und keine alternativlose Notwendigkeit darstelle. Vielmehr müsse eine überzeugende Wirtschaftsethik als ,eine Wirtschaftsethik ohne Reflexionsstopp vor irgendwelchen empirischen oder normativen Bedingungen der Marktwirtschaft ${ }^{\star 343}$ konzipiert werden. Aus diesen Überlegungen wird folglich deutlich, dass auch die Konzeption HOMANNS nach ULRICH systematisch in der ethischen Reflexion zu kurz greift. ${ }^{344}$

${ }^{340}$ Vgl. Ulrich, P. (2016a), S. 113; Ulrich, P. (1996), S. 2-3; Ulrich, P. (1994), S. 77; Ulrich, P. (1989), S. 20-21. In der Literatur wird dieser Gegenstandsbereich auch intensiv unter dem Begriff der „separation thesis“ diskutiert. Vgl. hierzu z. B. die kritischen Beiträge in Hartman, E. M. (2011); Freeman, R. E. et al. (2010); Dienhart, J. W. (2008); Harris, J. D. / Freeman, R. E. (2008); Wempe, B. (2008); Wicks, A. C. (1996); Freeman, R. E. (1994). ${ }^{341}$ Vgl. Ulrich, P. (2016a), S. 103-105; Ulrich, P. (2015a), S. 214-216; Ulrich, P. (2011b), S. 2-3; Ulrich, P. (2004b), S. 19-20; Ulrich, P. (1994), S. 80; Ulrich, P. (1989), S. 21-22.

342 Ulrich, P. (2016a), S. 135; Ulrich, P. (2015a), S. 216.

${ }^{343}$ Ulrich, P. (2016a), S. 133, in der Quelle ebenfalls herv.

${ }^{344}$ Vgl. Ulrich, P. (2016a), S. 118-123; Ulrich, P. (2004b), S. 20; Ulrich, P. (1994), S. 8081; Ulrich, P. (1989), S. 22-23. Vgl. auch die Überlegungen bei AßLÄNDER und NUTZINGER, welche pointiert in Abgrenzung zu HoMANN fordern: „Der systematische Ort der Moral ist die Ethik!“‘ (Aßländer, M. S. / Nutzinger, H. G. (2010), S. 226). 
Wie die vorigen Ausführungen darlegten, kritisiert ULRICH im Kern, dass bisherige wirtschaftsethische Ansätze eine faktische ökonomische Rationalität vielfach ungeprüft in ihre Konzeptionen übernommen hätten. Dagegen sei es gerade Aufgabe der Ethik, in einer vernunftgeleiteten Reflexion die wirtschaftliche Realität einer kritischen Würdigung zu unterziehen und damit die faktischen Normenverhältnisse in einem tiefergreifenden Ansatz selbst kritisch zu evaluieren. Wirtschaftsethik sei damit die „kritische Grundlagenreflexion der ökonomischen Vernunft“"345. In diesem Sinne setze die integrative Wirtschaftsethik ,vorbehaltlos grundlagenkritisch an, das heißt sie akzeptiert keine andere Vorgabe als die ethisch-kritische Reflexionsordnung selbst. “" ${ }^{446}$ Es geht dabei im Kern darum, „die ökonomische Rationalität selbst zur (praktischen) Vernunft zu bringen [...]. “347 Diese zentralen Überlegungen sowie die Abgrenzung zu den Konzeptionen von STEINMANN und LÖHR bzw. HOMANN fasst nochmals die nachfolgende Abbildung 3.12 zusammen.

Als zentralen Baustein greift ULRICH bei der Gestaltung seiner wirtschaftsethischen Konzeption auf klassische ethische Überlegungen in der Antike, insbesondere von ARISTOTELES, zurück. In Anlehnung an die aristotelischen Ausführungen im Rahmen der Nikomachischen Ethik sowie der Politik betont auch ULRICH, dass die Ethik als normative Reflexionstätigkeit den wirtschaftlichen oder politischen Realitäten überzuordnen sei. So folgt ULRICH auch konsequenterweise der ,aristotelische[n] Trias von Ethik, Politik und Ökonomik “348, wobei er hervorhebt, dass diese genau ,in dieser lexikalischen Ordnung ${ }^{\text {“349 }} \mathrm{zu}$ begreifen seien, d. h. die Ethik sei systematisch der politischen Reflexion und diese wiederum ökonomischen Überlegungen vorauszustellen. Es gilt damit nach ULRICH

${ }^{345}$ Ulrich, P. (2016a), S. 124. ULRICH bezeichnet seine Konzeption konsequent auch als „Vernunftethik des Wirtschaftens“ (Ulrich, P. (2016a), S. 125, in der Quelle ebenfalls herv.). Nach UlRICH kann sich eine solche ,Vernunftethik des Wirtschaftens“ dabei ,weder mit angewandter Ethik noch mit normativer Ökonomik begnügen, vielmehr geht es ihr im Kern gerade um einen integrativen Ansatz, der den ethischen Vernunftanspruch und den ökonomischen ,Rationalitätsanspruch ' buchstäblich ,zusammendenkt ‘ [...]." (Ulrich, P. (2016a), S. 101-102, in der Quelle ebenfalls herv.).

346 Ulrich, P. (2015a), S. 217.

${ }^{347}$ Ulrich, P. (2015a), S. 217.

348 Ulrich, P. (2015a), S. 218; Ulrich, P. (2003b), S. 154, in der Quelle ebenfalls herv. Vgl. ähnlich auch Ulrich, P. (2011a), S. 174 sowie grundlegend Ulrich, P. (2004a), S. 57-58.

${ }^{349}$ Ulrich, P. (2015a), S. 218; Ulrich, P. (2003b), S. 154. Vgl. auch Ulrich, P. (2011a), S. 174; Ulrich, P. (2006), S. 164. 


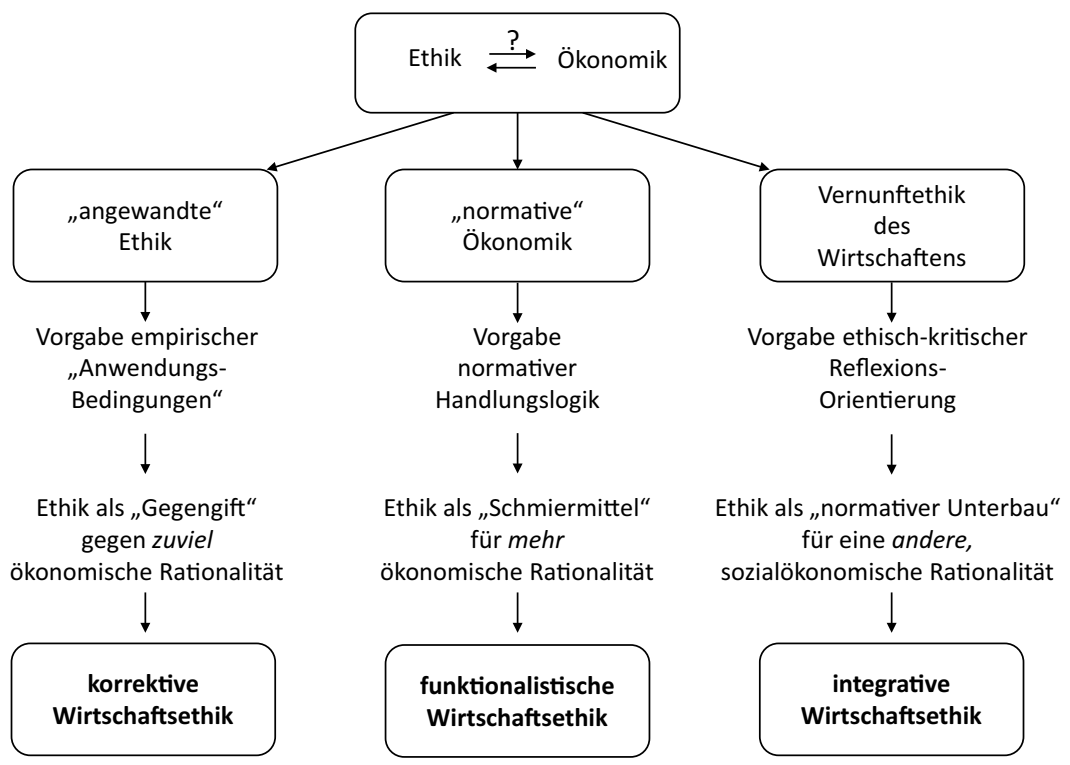

Abbildung 3.12 Positionierung der integrativen Wirtschaftsethik nach ULRICH ${ }^{350}$

das „Primat der Ethik“351. Ziel sei dabei durch eine systematische Grundlagenreflexion der wirtschaftlichen Realitäten und Überwindung einer scheinbaren Sachzwanglogik ${ }^{352}$ zu einer ,lebensdienlichen Wirtschaft“353 bzw. ,zivilisierten Marktwirtschaft“354 zu gelangen. Hierbei greift ULRICH wiederum die bereits

${ }^{350}$ Ulrich, P. (2015b), S. 216. Vgl. ähnlich auch Ulrich, P. (2016a), S. 135; Ulrich, P. (2013), S. 17; Ulrich, P. (2004b), S. 19.

${ }^{351}$ Ulrich, P. (2016a), S. 131. Vgl. auch Ulrich, P. (2011a), S. 167-169; Thielemann, U. / Ulrich, P. (2009), S. 40.

352 In diesem Sinne betont ULRICH: „Das Problem der systematischen Sachzwänge des marktwirtschaftlichen Wettbewerbs wird dabei als normatives Zumutbarkeitsproblem durchleuchtet, statt empirische Hindernisse [...] unhinterfragt in die Theoriekonstruktion zu übernehmen.“ (Ulrich, P. (2015a), S. 213-214, in der Quelle ebenfalls herv.).

353 Vgl. hierzu paradigmatisch Ulrich, P. (2016a), S. 11.

${ }^{354}$ Vgl. hierzu Ulrich, P. (2015a), S. 219; Ulrich, P. (2011c), S. 49; Ulrich, P. (2007), S. 5; Ulrich, P. (2004a), S. 71 sowie grundlegend auch die Ausführungen in Ulrich, P. (2016b); Ulrich, P. (2010). 
bei ARISTOTELES aufzufindende ${ }^{355}$ und auch in der katholischen Soziallehre intensiv rezipierte Forderung ${ }^{356}$ auf, dass im Sinne einer sozialökonomischen Rationalität ${ }^{357}$ die Wirtschaft nie als Selbstzweck aufzufassen sei, sondern lediglich als Mittel zur Erreichung eines guten Lebens in einer ,wohlgeordneten Bürgergesellschaft (civil society)“358 diene. ${ }^{359}$ In diesem Sinne sei auch die marktwirtschaftliche Ordnung nicht grundsätzlich legitimiert, sondern stets kritisch hinsichtlich ihrer Legitimität zu prüfen. ${ }^{360}$ Hierzu rekurriert ULRICH, wie auch STEINMANN und LÖHR, auf klassische diskursethische Überlegungen ${ }^{361}$, mit welchen alleinig die Legitimität von Normen zu prüfen seien, wobei im Gegensatz zu STEINMANN und LOHR bei ULRICH der Diskurs nicht (nur) zur fallspezifischen Konfliktlösung, sondern zur generellen Reflexion der herrschenden Wirtschaftsnormen und -praktiken angewandt wird.

Dabei weist die Konzeption, obschon sich ULRICH auch mit makro-, wie mesoethischen Betrachtungen befass ${ }^{362}$, eine starke individualethische Fundierung auf, denn im Rahmen seiner Konzeption nimmt der gebildete, aufgeklärte Wirtschaftsbürger ${ }^{363}$ als gemeinwohlorientierter citoyen eine zentrale Position ein. ${ }^{364}$ So sei ohne verstärkte wirtschaftsethische Bildungsmaßnahmen eine

\footnotetext{
${ }^{355} \mathrm{Vgl}$. in diesem Kontext auch die aristotelischen Überlegungen zur Differenzierung zwischen einer guten Hausbewirtschaftung bzw. Ökonomik (oíкоvo $\mu$ í $\alpha$ [oikonomía]) und einem

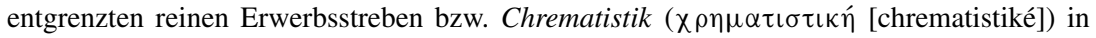
Aristoteles (2014), 1257a.

${ }^{356}$ So z. B. bei Nell-Breuning, O. v. (1992), S. 33-34. Vgl. hierzu auch die Anmerkungen bei Ulrich, P. (2016a), S. 11.

${ }^{357}$ Vgl. hierzu Ulrich, P. (2016a), S. 132; Ulrich, P. (2013), S. 15-16; Ulrich, P. (2011a), S. 173; Ulrich, P. (2004a), S. 63 und S. 70; Ulrich, P. (2003a), S. 157; Ulrich, P. (1994), S. $82-$ 85; Ulrich, P. (1989), S. 24.

${ }^{358}$ Ulrich, P. (2015a), S. 228, in der Quelle ebenfalls herv. Vgl. hierzu auch Ulrich, P. (2011b), S. 7; Ulrich, P. (2002), S. 48-49.

${ }^{359}$ Vgl. Ulrich, P. (2010), S. 11; Ulrich, P. (2004b), S. 13.

${ }^{360}$ Vgl. Ulrich, P. (2017), S. 12-13.

${ }^{361}$ Vgl. Ulrich, P. (2004d), S. 1. In diesem Sinne betont ULRICH, dass ,der systematische Ort der Moral" nicht, wie bei HOMANN, in der Rahmenordnung, sondern vielmehr ,in der unbegrenzten Öffentlichkeit aller Wirtschaftsbürger" (Ulrich, P. (1994), S. 104, in der Quelle ebenfalls herv.) aufzufinden sei.

362 Vgl. zum Überblick auch Nietsch-Hach, C. (2016), S. 92.

${ }^{363}$ Vgl. zum Begriff des Wirtschaftsbürgers auch detailliert Ulrich, P. (2007), S. 3-4.

364 Vgl. Ulrich, P. (2016a), S. 314 und S. 343; Ulrich, P. (2015a), S. 232; Ulrich, P. (2010), S. 71; Ulrich, P. (2004a), S. 62. In diesem Sinne betont auch BESCHORNER, dass UlRICHS Ansatz ,über eine Ethik der Wirtschaftsbürger entwickelt [wird].“ (Beschorner, T. (2013), S. 347).
} 
lebensdienlichere Wirtschaftsweise nicht zu erreichen, müssten doch die Bürger z. B. mit ihren Partizipationsrechten für eine Änderung der Wirtschaftsnormen eintreten bzw. einen verstärkten Fokus auf die Legitimität der Wirtschaftshandlungen legen und übereinstimmend hiermit konsistent handeln. ${ }^{365}$ In diesem Sinne gehe es folglich um die zentrale Bedeutung der Entwicklung bzw. Revitalisierung klassischer Bürgertugenden und die Schaffung eines republikanischen, $d$. $h$. gemeinwohlorientierten (Wirtschafts-)Bürgerethos. ${ }^{366}$ Denn schließlich sei auch ,auf demokratischem Weg buchstäblich kein liberaler Staat zu machen ohne BürgerInnen, die mehrheitlich eine gewisse moralische Orientierung mitbringen, nämlich einen gesunden Bürgersinn. “367

Hierauf aufbauend sei nicht nur verstärkte Verantwortung für das Gemeinwesen im staatlichen Sinne, sondern auch im Kontext unternehmerischen Handelns abzuleiten. So befasst sich ULRICH auch mit den Implikationen seiner integrativen Wirtschaftsethik auf der Mesoebene. Auch diese sei von besonderer Bedeutung, denn eine ,[g]ute Unternehmensführung erschöpft sich nicht im (sozial-)technisch und strategisch rationalen Mitteleinsatz für ,gegebene' Unternehmensziele und -zwecke, sondern setzt ethisch-praktisch vernünftige (d. h. legitime, also mit guten Gründen rechtfertigungsfähige) Zwecke und Grundsätze unternehmerischen Handelns voraus. “368 Die in diesem Zusammenhang entwickelte Unternehmensethik ist dabei grundsätzlich zweistufig aufgebaut. ${ }^{369}$ Auf der ersten Stufe der unternehmerischen Verantwortung (,Geschäftsethik“) ist stets kritisch zu prüfen, inwiefern das Unternehmen dem Zweck einer lebensdienlichen Wertschöpfungsaufgabe gerecht werde, wobei in diesem Zusammenhang, ähnlich wie in klassischen Beiträgen zur Stakeholder Theory, z. B. bei FREEMAN ${ }^{370}$, der Begriff der Wertschöpfung relativ breit im Sinne ,gesellschaftlich wertvoller“ bzw. legitimierter Tätigkeiten aufgefasst wird. ${ }^{371}$ Zentral ist hierbei nach ULRICH die „Suche nach rentablen Wegen sozialökonomisch sinnvollen und

\footnotetext{
365 Vgl. Ulrich, P. (2015a), S. 234.
}

366 Vgl. Ulrich, P. (2016a), S. 317; Ulrich, P. (2015a), S. 232; Ulrich, P. (2015b), S. 252253; Ulrich, P. (2011c), S. 51; Ulrich, P. (2008b), S. 334; Ulrich, P. (2007), S. 4-5; Ulrich, P. (2004a), S. 69; Ulrich, P. (2004b), S. 27.

${ }^{367}$ Ulrich, P. (2004a), S. 68, in der Quelle ebenfalls herv.

368 Ulrich, P. (2004d), S. 2, in der Quelle ebenfalls herv.

${ }^{369}$ Vgl. hierzu detailliert Ulrich, P. (2016a), S. 462-465; Ulrich, P. (2015b), S. 256-260; Ulrich, P. (2013), S. 28-29.

${ }^{370}$ Vgl. z. B. Freeman, R. E. (2017); Freeman, R. E. (2010b); Freeman, R. E. / Martin, K. / Parmar, B. (2007).

${ }^{371}$ Vgl. Ulrich, P. (2016a), S. 132; Ulrich, P. (2004c), S. 9-10; Ulrich, P. (1994), S. 84; Ulrich, P. (1989), S. 25. 
legitimen Wirtschaftens innerhalb der ordnungspolitischen Rahmenbedingungen [.].“372 Dabei sind jedoch auch organisationale Gestaltungsparameter zu berücksichtigen, welchen z. B. in den Ausführungen von HOMANN kaum Beachtung geschenkt wurde. So betont UlRICH in diesem Kontext beispielsweise: „Es gilt die Anreizstrukturen durchgängig so zu gestalten, dass ethisch verantwortungsvolles Handeln belohnt und rücksichtsloses, allein an persönlichen Bereicherungsoder Karrierezielen orientiertes Verhalten demotiviert wird statt umgekehrt.“373

Darüber hinaus wird dem Unternehmen aber auch in einer zweiten Stufe der Verantwortung (,republikanische Unternehmensethik“) eine Mitverantwortlichkeit bei der Gestaltung einer lebensdienlichen Wirtschaftsordnung zugesprochen, so dass hier wiederum eine Verbindung zur wirtschaftsethischen Makroebene deutlich wird. Da Unternehmen vielfach als ressourcenstarke Instanzen aufzutreten vermögen, sei diesen eine ,branchen- und ordnungspolitische Mitverantwortung “ 374 bei der Gestaltung der Wirtschaftsnormen aufgetragen, so z. B. bei der systematischen Analyse, in welchen Situationen wettbewerbsinduzierte Dilemmastrukturen problematisch erscheinen. Dabei seien, wenn möglich, auch Lösungsmöglichkeiten zu offerieren, wie solche Dilemmata möglicherweise zu überwinden wären, und diese wiederum in die politischen Entscheidungsarenen einzubringen. ${ }^{375}$ Unternehmen werden in diesem Sinne folglich nicht - wie z. B. bei RIEGER oder auch traditionellen Vertretern des Shareholder ValueParadigmas - mit dem Primärzweck einer Befriedigung der Ansprüche der Eigenkapitalgeber konzipiert, wobei alle anderen Ansprüche nur instrumentell, z. B. zur Steigerung des Eigenkapitalmarktwertes, berücksichtigt werden. ${ }^{376}$ Vielmehr vertritt ULRICH eine Auffassung ,der gesellschaftlichen Institution ,Unternehmung “ als pluralistischer Wertschöpfungsveranstaltung.“377 Diese haben als quasi-öffentliche Institutionen folglich einen Auftrag, die Ansprüche der Gesellschaft als Ganzes zu berücksichtigen ${ }^{378}$ und analog zum individuellen

372 Ulrich, P. (2016a), S. 465.

${ }^{373}$ Ulrich, P. (2016a), S. 494, in der Quelle ebenfalls herv.

${ }^{374}$ Ulrich, P. (2016a), S. 465, in der Quelle auch fett herv.

${ }^{375}$ Vgl. Ulrich, P. (2016a), S. 469-473; Ulrich, P. (2015b), S. 258; Ulrich, P. / Kaiser, M. (2001), S. 31; Ulrich, P. (1994), S. 100-102.

376 Vgl. hierzu auch die Ausführungen in Abschnitt 2.2.1.

377 Ulrich, P. (2016a), S. 430.

378 Vgl. Ulrich, P. (2016a), S. 489 sowie grundlegend auch Ulrich, P. (1977). Diese Überlegungen finden sich allerdings auch bei HOMANN, welcher hervorhebt: „Doch Unternehmen sind keine private, sondern eine gesellschaftliche Veranstaltung, von der Gesellschaft zur Erfüllung bestimmter Aufgaben eingerichtet und gesichert - zum Nutzen der Gesellschaft.“ (Homann, K. (2004), S. 7). 


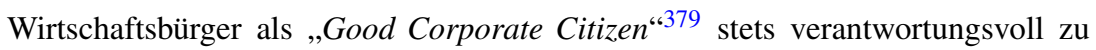
handeln und sich auch politisch gemeinwohlorientiert $\mathrm{zu}$ engagieren.

Zur Unterstützung dieser genuin republikanischen Orientierung sei jedoch schließlich auch die makroethische Ebene der Gestaltung einer anderen Wirtschaftsordnung $\mathrm{zu}$ bedenken, welche als , institutionelle Rückenstützen “380 die Zweckverfolgung lebensdienlichen Wirtschaftens sowohl auf der individual- als auch der mesoethischen Ebene unterstützen sollen, da andernfalls - und hier übernimmt ULRICH wiederum einen Gedanken HOMANNS - eine normative Überforderung der handelnden Akteure drohe: „Eine grenzenlose Moralzumutung an die Wirtschaftsakteure würde diese angesichts der systemischen Sachzwangstruktur der Marktwirtschaft systematisch überfordern.“"381 In diesem Sinne sei es daher ,durchaus nötig und sinnvoll, innerhalb einer grundsätzlich bereits legitim geordneten Marktwirtschaft das ethisch verantwortungsvolle Handeln aller Akteure durch die kluge Institutionalisierung funktionaler Anreize zu erleichtern und zu fördern." 382 Hierbei sei es auch besonders relevant, „,dafür [zu] sorgen, dass Trittbrettfahrer nicht belohnt werden, sondern verantwortliches, rücksichtsvolles Handeln begünstigt wird. “383 Dennoch seien im Gegensatz zu HoMANNS Ansatz weder Individuen noch Kollektive normativ vollständig zu entlasten, so dass bei diesen stets ein gewisses $\mathrm{Maß}$ an originärer ethischer Dispositionsverantwortung verbleibe. ${ }^{384}$ Da zudem, wie bereits hervorgehoben wurde, z. B. die Rahmenordnung in demokratisch-partizipativen Gesellschaften von den Bürgern bestimmt werde, verbleibe auch in diesem Handlungskontext bei diesen ein bedeutsames Maß an Letztverantwortung zur Gestaltung einer adäquaten, lebensdienliche Handlungen unterstützenden Rahmenordnung. ${ }^{385}$

\footnotetext{
${ }^{379}$ Ulrich, P. (2015b), S. 259, in der Quelle ebenfalls herv. Vgl. hierzu auch Ulrich, P. (2016a), S. 473 und S. 499; Ulrich, P. (2011c), S. 51; Ulrich, P. / Kaiser, M. (2001), S. 28-29; Ulrich, P. / Maak, T. (2000), S. 1.

${ }^{380}$ Ulrich, P. (2010), S. 98, in der Quelle ebenfalls herv. Vgl. hierzu auch Nietsch-Hach, C. (2016), S. 211; Thielemann, U. / Weibler, J. (2007a), S. 190.

${ }^{381}$ Ulrich, P. (2015a), S. 233.

382 Ulrich, P. (2017), S. 13.

${ }^{383}$ Ulrich, P. / Kaiser, M. (2001), S. 31.

384 Vgl. Ulrich, P. (2015a), S. 233.

385 Vgl. Ulrich, P. (2015a), S. 234.
} 


\subsubsection{Weitere neuere Entwicklungen der Wirtschaftsethik}

Die deutschsprachige Forschung hat neben den aufgezeigten drei klassischen wirtschaftsethischen Strömungen auch weitere Konzeptionen hervorgebracht, wobei im Folgenden vor allem der governanceethische Ansatz nach WIELAND, die kulturalistische Ethik von BESCHORNER und PFRIEM sowie die analytische Konzeption von KUPPER näher vorgestellt werden, da diese ebenfalls relativ verbreitet und umfassend ausgearbeitet sind.

\subsubsection{Der governanceethische Ansatz WielandS}

Die Konzeption WIELANDS beruht auf mehreren Theoriesträngen. ${ }^{386}$ So zieht WIELAND zur Begründung seiner Konzeption vor allem die soziologische Systemtheorie von LUHMANN, die Ressourcentheorie sowie die Neue Institutionenökonomik (und dort insbesondere die Transaktionskostentheorie ${ }^{387}$ ) heran. ${ }^{388}$ Dabei fokussiert WIELAND spezifisch auf Unternehmen, welche er in Anlehnung an LuHMANN als soziale Systeme begreift. Der Fokus auf Unternehmen sei hierbei gerechtfertigt, da diese in der globalisierten Wirtschaftswelt in immer größerem Maße als dominante Akteure durch fehlende bzw. auch potentiell unmögliche nationalstaatliche Regulierung aufträten: „Die Steuerungsdefizite nationalstaatlicher Rahmenordnungen und die Konkurrenz zwischen diesen führen dazu, dass den Unternehmen gesellschaftspolitische Verantwortung und ein

${ }^{386}$ Dabei grenzt sich WIELAND auch klar von der Konzeption ULRICHS ab und hebt beispielsweise hervor: „In der Wirtschaft gibt es schlicht keine moralischen Fragen, die in ihren Konsequenzen nicht auch wirtschaftliche Fragen sind. Einen Primat der Ethik kann man dann immer noch kommunizieren, aber nicht mehr durchsetzen." (Wieland, J. (2007), S. 31). Vgl. hierzu auch Wieland, J. / Ehrenberger, M. (2015), S. 117; Wieland, J. (2005b), S. 16; Wieland, J. (2001a), S. 25. Zur kritischen Rezeption der Kritik UlRICHS an WIELANDS Konzeption vgl. auch Wieland, J. (2007), S. 88-96. Dennoch existieren auch interessante konzeptionelle Parallelen. So gehe nach ULRICH auch ,die integrative Unternehmensethik vom Zwittercharakter moderner Unternehmen aus [..]: Einerseits sind sie Subsysteme des marktwirtschaftlichen Systems und müssen sich gemäß dessen ordnungspolitisch gewollter Funktionslogik im Wettbewerb behaupten; andererseits aber sind sie multifunktionale Wertschöpfungsinstitutionen, die mitten im Spannungsfeld einer Vielzahl je für sich legitimer, aber untereinander konfligierender und daher nach Fairness-Prinzipien zu berücksichtigender gesellschaftlicher (Stakeholder-)Ansprüche stehen." (Ulrich, P. (2008b), S. 335, in der Quelle ebenfalls herv.).

${ }^{387}$ Grundlegende Arbeiten auf diesem Gebiet finden sich bei WILLIAMSON (vgl. hierzu die Ausführungen bei Williamson, O. E. (1975); Williamson, O. E. (1973)). Vgl. auch die zentralen Überlegungen bei Coase, R. H. (1937).

388 Vgl. Wieland, J. / Ehrenberger, M. (2015), S. 116; Wieland, J. (2007), S. 9. 
Gestaltungsauftrag zugerechnet wird.“389 Aus diesem Grund sei konzeptionell bedeutsam, hervorzuheben, dass gerade durch den Prozess der Globalisierung Unternehmen ,in der entstehenden Weltökonomie und Weltgesellschaft zunehmend eine ökonomische und politische Steuerungsfunktion übernehmen.“390 Im Gegensatz zu LuHMANN, welcher generell die monolinguale Ausrichtung moderner, d. h. ausdifferenzierter sozialer Systeme postuliert, fordert WIELAND, Unternehmen als polylinguale Systeme aufzufassen ${ }^{391}$, welche gerade nicht nur in ökonomischen Größen operieren (müssen). In diesem Sinne betont WIELAND, „dass Unternehmen polylinguale Systeme sind. Anders als der Markt, der jedes Ereignis in Preisen codieren muss, um es kommunizieren zu können, müssen Unternehmen in der Lage sein, relevante Ereignisse in vielen Sprachspielen [...] gleichzeitig oder selektiv zu bewerten und zu verarbeiten. “392

Die Polylingualität sei zum Erhalt des Unternehmens von zentraler Bedeutung, beachte man die von den Stakeholdern an das Unternehmen gerichteten, vielfach auch außerökonomischen Erwartungen. Grundüberlegung der Konzeption von WIELAND ist dabei die Auffassung, dass Ethik nicht als exogenes Element von wirtschaftlichen Transaktionen, sondern vielmehr als integraler Bestandteil dieser Austauschprozesse aufzufassen sei. Moral kann in diesem Falle (ähnlich wie auch bei PIES) als Produktionsfaktor aufgefasst werden bzw. repräsentiert ein ,endogenes Element effizienter Ressourcenallokation.“393 Grundgedanke ist folglich, dass Ethikmaßnahmen im ökonomischen Sinne als Möglichkeit zur Reduktion von Transaktionskosten aufgefasst werden können, welche durch Unsicherheit bei ökonomischen Transaktionen resultieren. ${ }^{394}$ Zentral ist hierbei „die Absicht, stabile Handlungserwartungen und Kontrolle über die Organisations- und Gesellschaftsumwelt des Unternehmens dadurch zu erreichen, dass man sich selbst bindet und damit Anreize für andere schafft, sich auch zu binden.“" ${ }^{395}$ So spiele

\footnotetext{
${ }^{389}$ Wieland, J. (2007), S. 24. Vgl. hierzu auch Wieland, J. (2005b), S. 15; Wieland, J. I Grüninger, S. (2000), S. 160-161.

390 Wieland, J. (2007), S. 13.

${ }^{391}$ Vgl. Wieland, J. / Ehrenberger, M. (2015), S. 126-127; Wieland, J. (2014), S. 3334; Wieland, J. (2007), S. 40 und S. 63; Wieland, J. (2005b), S. 58; Wieland, J. (2004b), S. 8; Wieland, J. (2001b), S. 79. Diese Auffassung divergiert folglich von der klassischen systemtheoretischen Auffassung nach LUHMANN und ist deshalb in der Literatur in dieser Hinsicht auch kritisch rezipiert worden. Vgl. hierzu z. B. Quandt, J. H. (2013), S. 86-87.

392 Wieland, J. (2007), S. 63-64. Vgl. ähnlich auch Wieland, J. (2001b), S. 79.

${ }^{393}$ Wieland, J. (2007), S. 9.

394 Vgl. Wieland, J. / Ehrenberger, M. (2015), S. 130; Wieland, J. (2001a), S. 21-22.

395 Wieland, J. (2007), S. 68.
} 
auch die Zuverlässigkeit bzw. moralische Integrität von Akteuren eine bedeutsame Rolle im Wirtschaftskontext, welche von WIELAND als Ressource aufgefasst wird. ${ }^{396}$ Dabei geht es, ähnlich wie auch bei SUCHANEK, im unternehmerischen Kontext um eine „Stabilisierung vorhandener und Generierung neuer Handlungsmöglichkeiten, also Kooperationschancen [...]." ${ }^{\text {397 }}$ Moral wird in diesem Sinne folglich ,als Bedingungsgarant gelingender ökonomischer Transaktionen verstanden." ${ }^{398}$ Um eine solch verbesserte ökonomische Interaktion zu gewährleisten, sei die Entwicklung und kritische Durchdringung von potentiellen Steuerungsbzw. Governancemechanismen ${ }^{399}$ auf unternehmerischer Ebene zentrale Aufgabe einer wissenschaftlichen Unternehmensethik als Governanceethik, wodurch sich neben deskriptiven Elementen auch ein analytischer Fokus ${ }^{400}$ der Konzeption ergibt. Zusammenfassend gilt für die Governanceethik nach WIELAND die folgende Definition: „Die Governanceethik des Unternehmens ist die Lehre von der komparativen Analyse der moralsensitiven Gestaltung und Kommunikation der Governancestrukturen spezifischer wirtschaftlicher Transaktionen mittels Kooperationen. “401

Die Möglichkeit einer wirksamen Umsetzung moralischer Ansprüche in ökonomischen Transaktionen kann nach WIELAND durch vier Parameter dargestellt werden. So spielen zum einen individuelle Selbstbindungsstrategien (IS), repräsentiert z. B. durch Tugenden oder organisationale Werte, eine bedeutsame Rolle. Darüber hinaus sind nach WIELAND auch formale Institutionen (FI),

${ }^{396}$ Vgl. Wieland, J. / Ehrenberger, M. (2015), S. 116-117. Vgl. hierzu auch Wieland, J. (2005a), S. 261; Wieland, J. (2004b), S. 12; Wieland, J. (2001b), S. 75.

397 Wieland, J. (2007), S. 40. Vgl. auch Wieland, J. (2001a), S. 13. Die Parallele zu SuCHANEK wird in diesem konzeptionellen Detail auch durch die Bezeichnung unternehmensethischer Maßnahmen als „Investition“ (Wieland, J. (2007), S. 67) bzw. zu HomanN im Allgemeinen durch die Hervorhebung der Bedeutung einer ,anreizsensitive[n] Implementierung in die Governancestrukturen wirtschaftlicher Transaktionen“ (Wieland, J. (2007), S. 95) deutlich. Vgl. hierzu auch Wieland, J. / Ehrenberger, M. (2015), S. 114.

398 Wieland, J. (2007), S. 9.

${ }^{399}$ Nach WIELAND wird unter Governance „eine Steuerungsstruktur oder eine Steuerungsmatrix zur Abwicklung wirtschaftlicher und gesellschaftlicher Transaktionen“ (Wieland, J. (2007), S. 13) verstanden.

${ }^{400}$ Vgl. hierzu Wieland, J. / Ehrenberger, M. (2015), S. 114. Der Begriff ,,analytisch“ unterscheidet sich jedoch im Bezug zur Auffassung KUPPERS darin, dass ein geringerer Fokus auf praktisch-normative Begründungszusammenhänge gelegt wird. Vgl. hierzu auch die Ausführungen zur Konzeption KÜPPERS in Abschnitt 3.4.5.3.

${ }^{401}$ Wieland, J. (2007), S. 75. Vgl. auch Wieland, J. (2001a), S. 16. 
wie z. B. staatliche Regulierung und privatwirtschaftliche Branchenvereinbarungen sowie informale Institutionen (IF), bspw. gesellschaftlich existierende, implizite Wertvorstellungen, zu nennen. Abschließend seien jedoch auch organisationale Koordinations- und Kooperationsmechanismen (OKK), wie z. B. unternehmensinterne Codes of Conduct oder Ethikchecklisten, von besonderer Relevanz, wobei gerade hierauf aus governanceethischer Perspektive ein besonderer Fokus läge. ${ }^{402}$ Diese Überlegungen werden von WIELAND nochmals formal in der s. g. Governanceformel dargestellt, welche die Realisierung der moralischen Komponenten in einer ökonomischen Transaktion als Funktion der genannten Einflussfaktoren ${ }^{403}$ darstellt: $^{404}$

$$
T_{m i}=f\left(a I S i j, b F I_{i j}, c I F_{i j}, d O K K_{i j}\right)
$$

Abschließend befasst sich WIELAND auch mit der Implementierung der Governancefragen in der unternehmerischen Praxis, wobei er einen starken Fokus auf die praxisnahe Entwicklung von Ethik- bzw. Wertemanagementsysteme legt, welche er konzeptionell erarbeitet. ${ }^{405}$ In diesem Kontext betont WIELAND zudem die Bedeutung der organisationsinternen Förderung von Moral mittels adäquater Anreizsysteme, welche jedoch nicht nur monetär incentivieren sollten. Nach

402 Vgl. Wieland, J. / Ehrenberger, M. (2015), S. 118-120; Wieland, J. (2008a), S. 305-306; Wieland, J. (2001a), S. 8-11.

${ }^{403}$ WIELAND fasst diese Faktoren in einem tugendethischen Sinne auch als Ermöglichungsfaktoren auf, da diese potentiell gute individuelle wie kollektive Intentionen zusätzlich institutionell unterstützten. Vgl. Wieland, J. (2006), S. 7-8; Wieland, J. (2005b), S. 76 und S. 83-84; Wieland, J. (2004b), S. 10.

${ }^{404} \mathrm{~T}_{\mathrm{m}}$ bezeichnet hierbei die moralische Komponente einer ökonomischen Transaktion, $\mathrm{i}$ eine spezifische Transaktion und $\mathrm{j}$ den spezifischen Kontext. Die Parameter a, b, c und d repräsentieren den gegenwärtigen positiven $(=+1)$, neutralen $(=0)$ oder negativen $(=-1)$ Einfluss der jeweiligen Komponente auf die Verwirklichung der moralischen Ansprüche in einer Transaktion. Vgl. hierzu detailliert Wieland, J. / Ehrenberger, M. (2015), S. 119; Wieland, J. (2008a), S. 307-308; Wieland, J. (2005b), S. 29-31; Wieland, J. (2004a), S. 3; Wieland, J. (2004b), S. 11; Wieland, J. (2001a), S. 9-11.

405 Vgl. Wieland, J. / Ehrenberger, M. (2015), S. 134-140; Wieland, J. (2008b), S. 15-17; Wieland, J. (2007), S. 97-111; Wieland, J. (2004a), S. 17-20; Wieland, J. (2004c), S. 2327; Wieland, J. (2003), S. 13-22; Wieland, J. (2002), S. 4-6; Wieland, J. / Grüninger, S. (2000), S. 160-166. Vgl. hierzu auch die empirischen Ergebnisse anhand eines praktischen Fallbeispiels in Wieland, J. / Fürst, M. (2003). 
WIELAND sind Unternehmen daher gefordert, ,ein umfassendes Anreizmanagement auf[zu]bauen, das sich nicht auf ökonomische Anreize reduzieren lässt““406 und z. B. auch soziale Wertschätzung als extrinsischen Anreiz aufweist. ${ }^{407}$

\subsubsection{Die kulturalistische Ethik Beschorners und Pfriems}

Ein Ansatz, der gewisse Ähnlichkeiten mit den Überlegungen WIELANDS aufweist, in Teilen aber auch über diese hinausgeht, stammt von BESCHORNER und PFRIEM, welche eine kulturalistische Wirtschaftsethik konzipieren. Ausgangspunkt dieser Überlegungen ist, dass das Phänomen der Kultur in den bisherigen wirtschaftsethischen Konzeptionen zu wenig Beachtung gefunden habe. In diesem Kontext stellt BESCHORNER zudem fest, „dass sich in der ökonomischen Praxis eine zunehmende Relevanz von Kultur feststellen lässt. Kultur versteht sich dabei nicht als ein Gegenstandsbereich (Kulturmanagement o.ä.), sondern als eine Dimension des menschlichen Lebens, die alle Lebensbereiche durchzieht $^{408}$ - auch die Ökonomie. “409 Hierbei sei auch zentral, dass der Ansatz durch seinen kulturalistischen Fokus auch nicht-ökonomische Faktoren hervorhebt, welche gleichsam einen Einfluss auf ökonomische Prozesse ausübten. ${ }^{410}$ Diese Überlegungen folgen dem grundlegenden Programm einer „Aufklärung durch Perspektivenpluralität “411, welches zu einem besseren Verständnis der realweltlichen wirtschaftlichen Handlungen führen soll und welches hervorhebt, dass der

406 Wieland, J. (2007), S. 64. Vgl. hierzu auch Wieland, J. (2005b), S. 115; Wieland, J. (2004b), S. 12; Wieland, J. (2001b), S. 80.

407 Vgl. Wieland, J. (2005b), S. 122; Wieland, J. (2004b), S. 15-16 und S. 20-21.

${ }^{408}$ Der Begriff der Kultur ist dabei überaus schillernd. Eine klassische Definition von Kultur stammt hierbei von HOFSTEDE, welcher diese als ,collective programming of the mind“ (Hofstede, G. (2001), S. 1) auffasst und kulturübergreifend in einer empirischen Studie clusteranalytisch fünf zentrale Dimensionen der Kultur extrahiert (vgl. Hofstede, G. (2001), S. 29). Eine weitere bekannte Definition stammt von GEERTZ, welcher Kultur als ,webs of significance" (Geertz, C. (1973), S. 5) auffasst. Vgl. im organisationalen Kontext auch die Ausführungen bei Schein, E. H. / Schein, P. (2017), S. 3-5; Hofstede, G. (2001), S. 391-393 sowie die Ausführungen in Abschnitt 4.7.

${ }^{409}$ Beschorner, T. (2013), S. 350. Vgl. ähnlich auch Beschorner, T. / Hajduk, T. / Schank, C. (2012), S. 87; Beschorner, T. / Nutzinger, H. G. (2007), S. 224. In diesem Sinne postuliert PFRIEM: „Eine kulturalistische oder kulturwissenschaftliche Perspektive liegt im Trend“ und sei zur Zeit im wissenschaftlichen Diskurs „en vogue“ (Pfriem, R. (2007a), S. 65). Auch LUDDEMANN stellt allgemein fest: „Kultur hat [...] Konjunktur.“ (Lüddemann, S. (2019), S. 1). Zur Bedeutung von Kultur im Wirtschaftskontext, insbesondere auch in der Postmoderne, vgl. auch die Überlegungen bei Koslowski, P. (1989), S. 13-16. Vgl. auch Osterloh, M. (1991), S. 154-156.

${ }^{410}$ Vgl. Beschorner, T. (2013), S. 352.

${ }^{411}$ Beschorner, T. (2013), S. 351. 
neoklassische Ansatz nur einen möglichen Zugang zum Verständnis wirtschaftlicher Zusammenhänge darstelle. ${ }^{412}$ So wird auch von PFRIEM die methodische Trennung von Ökonomik und Ethik kritisiert, da kulturelle Praktiken schon immer normativ aufgeladen seien, welches der neoklassische Rigorismus jedoch aus seinen Modellen exkludiere und damit zu kurz greife. ${ }^{413}$

Die Anknüpfungspunkte eines kulturalistischen Programms lassen sich dabei anhand mehrerer Ebenen verdeutlichen. ${ }^{414}$ So wird z. B. auf der Ebene der Handlungen der ,rational choice“"415 -Ansatz sowie das diesem zugrunde liegende Leitbild des Homo oeconomicus als Handlungsparadigma verworfen und durch ein umfassenderes Modell ersetzt. Ferner wird betont, dass Interaktionen nicht nur als (ökonomisch motivierter) Äquivalenztausch aufzufassen seien, welches nur eine Teilmenge reziproker Handlungen darstelle. Hinsichtlich der Institutionen wird zudem nicht nur auf extrinsische Anreize fokussiert, sondern Institutionen werden im klassischen soziologischen Sinne ,,als komplexitätsreduzierende Formen zur Gewährleistung von Erwartungssicherheit ${ }^{\star 416}$ erachtet. Hinsichtlich der Organisationstheorie folgt die kulturalistische Ethik wiederum der Auffassung WIELANDS, dass Organisationen als polylinguale Systeme zu konzipieren seien. Schließlich wird in diesem Kontext hervorgehoben, dass auch die Ökonomie als gesellschaftliches Teilsystem nicht nur einer monetären Monologik folge. ${ }^{417}$

Wie diese Ausführungen bereits darlegten, zeigen sich im Vergleich zur Konzeption WIELANDS einige Parallelen, insbesondere im Kontext der postulierten Polylingualität von Organisationen. So sei auch ,,[a]us der Organisationstheorie [.] hinlänglich bekannt, dass Unternehmen keine einfachen, mechanischen Systeme sind, in denen nur die Sprache der Ökonomie gesprochen wird, sondern [, dass] es sich bei ihnen um komplexe soziale Systeme handelt, bei denen eine Vielzahl von Handlungsroutinen und -orientierungen zum Tragen kommen [...]. “418 In diesem Sinne bauen die Überlegungen BESCHORNERS und PFRIEMS in Teilen auf der Konzeption WIELANDS auf, wobei sie diese im Rahmen einer kulturalistischen Betrachtung jedoch $\mathrm{zu}$ erweitern intendieren. Wie z. B. PFRIEM hervorhebt, geht es der kulturalistischen Wirtschaftsethik folglich

\footnotetext{
412 Vgl. Beschorner, T. (2013), S. 351.

413 Vgl. Pfriem, R. (2015), S. 192.

414 Vgl. Beschorner, T. (2013), S. 352-353.

${ }^{415} \mathrm{Vgl}$. hierzu einführend z. B. die Ausführungen in Rosa, H. / Strecker, D. / Kottmann, A. (2018); Saalmann, G. (2016); Meleghy, T. (2015); De Jonge, J. (2012); Braun, N. (2009).

416 Beschorner, T. (2013), S. 352-353.

417 Vgl. Beschorner, T. (2013), S. 359-360.

418 Beschorner, T. (2013), S. 359.
} 
um ,eine kulturwissenschaftliche Weiterentwicklung der Governanceethik“419. Zudem sei die kulturalistische Ethik, wie auch bei WIELAND, ,in einem ersten Schritt nicht normativ, sondern als sozialwissenschaftlich - genauer: kulturwissenschaftlich - verstehend, beschreibend und erklärend $\mathrm{zu}$ betrachten und will über eine interpretative Handlungs- und zugleich Institutionentheorie zu , dichteren Beschreibungen ' $(G e e r t z)^{420}$ und einem ,Mehr' an Erklärungen gegenüber der traditionellen ökonomischen Lehre gelangen."421 Über diese vergleichbaren konzeptionellen Grundlagen hinaus zeigen sich aber auch Unterschiede zur Auffassung WIELANDS. Neben der Kultur, statt Governancestrukturen als Ausgangspunkt wirtschaftsethischer Betrachtung, werden so z. B. Organisationen im Kontext der kulturalistischen Wirtschaftsethik auch ,nicht durch einen (erweiterten) Transaktionskostenansatz charakterisiert, sondern als Ressourcenbündel, als ein spezifisches Set individueller und organisationaler Wissensbestände und Fähigkeiten (capabilities) betrachtet." 422

Zusätzlich zu diesen grundlegenden konzeptionellen wirtschaftsethischen Überlegungen haben sich BESCHORNER und PFRIEM auch mit den Implikationen auf der Mesoebene beschäftigt, für welche sie ebenfalls die Notwendigkeit eines „,cultural turn“ ${ }^{423}$ herausarbeiten. Auf konzeptioneller Ebene fordern sie dabei eine Abkehr vom neoklassisch-mathematischen Formalismus in der Beschreibung betrieblicher Tätigkeiten und eine Hinwendung zur Kultur als zentralem Einflussfaktor in Unternehmen. ${ }^{424}$ In diesem Sinne wird Unternehmensethik zum einen klassisch zwischen der mikroethischen Individualebene sowie der makroethischen Ordnungsethik verankert. ${ }^{425}$ Zum anderen wird jedoch, ähnlich wie wiederum bei WIELAND, die Bedeutung der institutionellen Unterstützung auf organisationaler Ebene angemahnt: ,Eine moderne Unternehmensethik begnügt sich nicht damit, lediglich an das Gute im Menschen zu appellieren [...]. Ihr geht es vielmehr um die Schaffung institutioneller Arrangements in der Organisation, in deren Rahmen

\footnotetext{
${ }^{419}$ Pfriem, R. (2005), S. 204. Vgl. zudem die Überlegungen auf S. 184 sowie S. 209.

${ }^{420}$ Vgl. hierzu die Ausführungen bei Geertz, C. (1973).

${ }^{421}$ Beschorner, T. (2013), S. 350. Vgl. ähnlich auch Beschorner, T. / Hajduk, T. / Schank, C. (2012), S. 87. Vgl. hierzu auch die weiteren Ausführungen bei BESCHORNER, in welchen dieser nochmals hervorhebt, dass es bei ,dem hier skizzierten Vorschlag einer wirtschaftsethischen Theorie der Anwendung nicht um die Entwicklung einer normativen (Wirtschafts-) Ethik geht.“ (Beschorner, T. (2013), S. 366).

422 Beschorner, T. (2013), S. 360.

${ }^{423}$ Pfriem, R. (2008b), S. 70. Vgl. hierzu auch die grundlegenden Überlegungen bei Hanekamp, G. (2001).

${ }^{424}$ Vgl. Pfriem, R. (2015), S. 191.

${ }^{425}$ Vgl. Beschorner, T. (2008), S. 86.
} 
moralisches Handeln stattfinden kann [.]."426 Auch in diesem Kontext folgen die Autoren prinzipiell wieder den Überlegungen WIELANDS, welcher seine Konzeption als tugendethisch geprägt klassifiziert. So betonen auch BESCHORNER und PFRIEM im organisationalen Kontext die Relevanz von Tugenden ${ }^{427}$ als „moralische Gestaltungskräfte. Sie befähigen einen Akteur (eine Unternehmung), in einem komplexen Feld moralischer Ansprüche und ungewisser Ergebnisse zu handeln. "428 Hierbei seien klassische universalistische Ethiken einer vielschichtigen, kontextsensitiven und „,bunten“"429 Wirklichkeit der Moderne nicht mehr gewachsen, weshalb PFRIEM zu dem Schluss kommt, sich mit aller Nachdrücklichkeit „,von der klassisch abendländischen Pflichtenethik zu verabschieden.““30 Insbesondere sei auch in der modernen, globalisierten Wirtschaftswelt eine zunehmende kulturelle Sensibilität von entscheidender Bedeutung für einen dauerhaften Unternehmenserfolg, welche in diesem Kontext auch als ,kulturelle Kompetenz"431 bezeichnet wird. In diesem Sinne schlussfolgert PFRIEM subsumierend: „Unsere mögliche Moral heißkt kulturelle Bildung. "432 Dabei hebt BESCHORNER abschließend jedoch auch hervor, dass eine reine legalistische Regelkonformität noch nicht als hinreichend $\mathrm{zu}$ erachten wäre, da vielmehr ein umfassenderes Integritätsmanagement von Bedeutung sei. ${ }^{433}$

\footnotetext{
${ }^{426}$ Beschorner, T. (2008), S. 89.

427 Auch LAUTERMANN und PFRIEM betonen eine gegenwärtige „Renaissance der Tugendethik“. Vgl. hierzu Pfriem, R. (2015), S. 204 sowie detailliert Lautermann, C. / Pfriem, R. (2006).

${ }^{428}$ Pfriem, R. (2008b), S. 68. Zur Relevanz einer Unterstützung moralischen Handelns durch institutionelle Stimuli vgl. auch Beschorner, T. (2013), S. 359.

${ }^{429}$ So betont auch BESCHORNER im Kontext der realen Handlungspraxis prägnant: „Die Welt ist nicht weiß, sie ist aber auch nicht schwarz. Die Welt ist bunt." (Beschorner, T. (2013), S. 366).

${ }^{430}$ Pfriem, R. (2008b), S. 66. In diesem Sinne postuliert PFrIEM auch, ,dass die Situiertheit und Kontextgebundenheit jeder ethisch-moralischen Entscheidungssituation nicht hintergangen werden kann“, so dass sich eine „Unbedingtheit über alle historisch und kulturell differenten Konstellationen und Konfigurationen hinweg [..] nach dem hier vertretenen Standpunkt nicht länger aufrechterhalten [lässt].“ (Pfriem, R. (2008b), S. 66).

${ }^{431}$ Pfriem, R. (2005), S. 193. Diese Kompetenzen seien zudem als „Fähigkeiten individueller wie kollektiver Akteure, aktiv an der Veränderung von Gesellschaft mitzuwirken“ (Pfriem, R. (2015), S. 198) von entscheidender Bedeutung.

432 Pfriem, R. (2015), S. 199, in der Quelle ebenfalls herv. Vgl. hierzu auch die detaillierten Überlegungen in Pfriem, R. (2007b).

${ }^{433}$ Vgl. Beschorner, T. (2008), S. 91-92. Vgl. hierzu auch die konzeptionellen Überlegungen bei Noll, B. (2013), S. 185-187; Paine, L. S. (1994).
} 


\subsubsection{Die analytische Ethik KüPPERS}

Einen anderen konzeptionellen Zugang, welcher grundlegend auch für die weiteren Überlegungen dieser Arbeit besonders fruchtbar erscheint, stammt von KUPPER. Zentraler Gedanke ist hierbei mittels analytischen Fokus ethische Probleme im betrieblichen Kontext zu entdecken und einer systematischen Lösung zuzuführen, so dass sich ein starker Fokus auf eine Entscheidungsunterstützung des Managements ergibt. KÜPPERS Konzeption ist dabei als Unternehmensethik klar auf der wirtschaftsethischen Mesoebene ${ }^{434}$ zu verankern. ${ }^{435}$ In diesem Sinne definiert KÜPER den Gegenstandsbereich der analytischen Ethik $^{436}$ wie folgt: „Die analytische Unternehmensethik ist darauf gerichtet, ethisch relevante Fragestellungen in Unternehmungen zu untersuchen. Sie soll Probleme von Unternehmungen aufgreifen, für deren Entstehung und Bewältigung ethische Bezüge von Bedeutung sind, deren Struktur herausarbeiten und Ansätze zu ihrer Lösung aufzeigen. “437 Dies sei besonders für eine anwendungsorientierte Disziplin wie der Betriebswirtschaftslehre von Bedeutung, denn diese ,sollte auch Hilfestellungen zur Analyse und Lösung von Wertfragen leisten [...].“438

KUPPERS analytisch ausgerichtete Konzeption beginnt dabei, wie auch z. B. bei STEINMANN und LÖHR, mit der Feststellung, dass Unternehmen in der heutigen Weltwirtschaft über steigende Handlungsspielräume verfügten, welches wiederum mit einer erhöhten Verantwortung einhergehe. Ferner sei grundsätzlich eine rein legalistische Handlungssteuerung wirtschaftlicher Aktivitäten unmöglich und zudem aus Gründen des Wettbewerbs unpraktikabel: „Die Existenz von Handlungsspielräumen ist damit nicht nur eine Folge einer unvermeidbaren Unvollständigkeit der Rahmenordnung, sondern eines ihrer konstitutiven Elemente.“439 Zunehmende Wichtigkeit erhalte dabei die solide Analyse von ethischen Problemstellungen, welche sich durch wirtschaftliche Handlungen von

\footnotetext{
${ }^{434}$ In diesem Sinne ist nach KÜPPER der Gegenstand der analytischen Unternehmensethik die „Untersuchung ethischer Fragestellungen bei wirtschaftlichen Entscheidungen in Unternehmungen“ (Küpper, H.-U. (2011), S. 34, in der Quelle fett herv.). Sie befasst sich ,,mit der Analyse, Begründung, Anwendung und Folgen von Normen, Werten sowie Werturteilen bei wirtschaftlichen Entscheidungen.“ (Küpper, H.-U. (2011), S. 35).

435 Vgl. Küpper, H.-U. (2011), S. 169.

${ }^{436} \mathrm{Vgl}$. zur konzeptionellen Bedeutung des Terminus ,analytisch“ auch die Ausführungen in Küpper, H.-U. / Schreck, P. (2009), S. 27.

${ }^{437}$ Küpper, H.-U. (2011), S. 169. Vgl. ähnlich auch Küpper, H.-U. (2007), S. 257.

${ }^{438}$ Küpper, H.-U. (2015), S. 320. Vgl. auch Küpper, H.-U. (2015), S. 326; Küpper, H.-U. I Schreck, P. (2009), S. 13.

${ }^{439}$ Küpper, H.-U. (2011), S. 36, in der Quelle fett herv. Vgl. hierzu auch Küpper, H.-U. (2015), S. 326; Küpper, H.-U. / Schreck, P. (2009), S. 25-26.
} 
Unternehmen ergäben, wobei insbesondere die Manager in immer größerem Maße argumentativ gefordert seien. Die analytische Ethik setzt hierbei an, indem sie ein Raster entwickelt, welches der Detektion wirtschaftsethischer Probleme im betrieblichen Kontext dient. Innerhalb dieses Rasters wird so, analog zu KÜPPERS Controllingkonzeption ${ }^{440}$, zwischen Leistungs- und Führungssystem (funktional) differenziert, wobei auch Governancefragen (und damit prinzipiell die Überlegungen WIELANDS) als drittes Problemfeld und übergreifende Steuerungsarchitektur in Unternehmen Berücksichtigung finden. ${ }^{441}$ In diesem Zusammenhang werden nun einige Felder systematisch erarbeitet. Dabei werden etwa ethische Fragestellungen u. a. im Kontext von Verantwortung, den Werten sowie dem organisationalen Zielsystem oder der Personalführung diskutiert. Auf der Ebene des Leistungssystems erfolgt wiederum eine Gliederung nach Produktion, Marketing und Investition. Diese sicherlich nicht als abschließend zu bezeichnende Klassifikation wird nun hinsichtlich ethischer Problemstellungen in verschiedenen Analyseebenen evaluiert. So werden in einem ersten Schritt relevante unternehmensethische Fragestellungen in den oben aufgezeigten betrieblichen Funktionen herausgearbeitet. Hierbei wird bei KÜPPER ein dominanter Fokus auf (v. a. individuelle) Werte als zentrale Einflussfaktoren des Handelns gelegt. In einem zweiten Schritt werden die Wirkungen dieser Werte auf organisationale Zielsetzungen beleuchtet, welche in einem dritten Schritt auf mögliche Beziehungen und Konflikte analysiert werden, wobei ein besonderer Fokus auf ethisch-ökonomischen Konflikten liegt. ${ }^{442}$ Gerade letztere seien durch die Globalisierung und potentiell konfligierenden Wertesystemen, welchen sich weltweit operierende Unternehmen gegenübergestellt sähen, von entscheidender Bedeutung in der Analyse. ${ }^{443}$ Abschließend muss dann in einem vierten Schritt überzeugend begründet werden, welchen dieser Werte gefolgt werden soll. ${ }^{444}$ Dieses Schema stellt nochmals folgende Tabelle 3.1 dar.

Eine solche Entscheidung ist dabei normativ, d. h., sie beruht auf einer korrespondierenden „Sollens“-Aussage, die wie bereits einleitend aufgezeigt wurde, einer überzeugenden Begründung bedarf. Einer solch wissenschaftlich fundierten Begründung widmet sich KÜPER detailliert. So schlägt dieser beruhend

\footnotetext{
${ }^{440}$ Vgl. hierzu Küpper, H.-U. et al. (2013), S. 36.

441 Vgl. Küpper, H.-U. (2011), S. 178-179.

${ }^{442}$ Vgl. Küpper, H.-U. (2011), S. 173; Küpper, H.-U. (1999), S. 63.

443 Vgl. Küpper, H.-U. (2011), S. 174.

${ }^{444}$ Vgl. detailliert Küpper, H.-U. (2016), S. 16-17; Küpper, H.-U. (2015), S. 328-331; Küpper, H.-U. (2011), S. 172-174 und S. 176-179; Küpper, H.-U. (2009), S. 788-789; Küpper, H.-U. / Schreck, P. (2009), S. 28; Küpper, H.-U. (2007), S. 262-264.
} 
Tabelle 3.1 Analytisches Evaluationsframework nach KUPPER ${ }^{445}$

\begin{tabular}{|c|c|c|c|c|c|c|c|c|c|}
\hline \multirow{2}{*}{$\begin{array}{l}\begin{array}{l}\text { Anwendunes } \\
\text { bereche }\end{array} \\
\begin{array}{l}\text { Analse- } \\
\text { dimensionen }\end{array}\end{array}$} & \multirow{2}{*}{$\begin{array}{l}\begin{array}{l}\text { Corporterte } \\
\text { Gover- } \\
\text { nance }\end{array} \\
\end{array}$} & \multicolumn{5}{|c|}{ Fürungessystem } & \multicolumn{3}{|c|}{ Leistunessystem } \\
\hline & & $\begin{array}{l}\text { Enstcheidung } \\
\text { Cund } \\
\text { Verantwortung }\end{array}$ & $\begin{array}{l}\text { Werto-und } \\
\text { Delesstem }\end{array}$ & 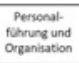 & 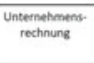 & Controling & Production & Marketing & $\begin{array}{l}\text { Investition und } \\
\text { Finnoniterung }\end{array}$ \\
\hline \multicolumn{10}{|l|}{$\begin{array}{l}\text { Unternehmensethische } \\
\text { Fragestellungen }\end{array}$} \\
\hline \multicolumn{10}{|l|}{ Wirkunesanalyse } \\
\hline \multicolumn{10}{|l|}{$\begin{array}{l}\text { Beriehungs- und } \\
\text { Konfistanulyse }\end{array}$} \\
\hline Begündunesanalse & & & & & & & & & \\
\hline & & & & & & & & & \\
\hline
\end{tabular}

auf seinen grundlegenden wissenschaftstheoretischen Überlegungen vor, konkrete Werthaltungen durch die Rückbindung an anerkannte Basiswertungen ${ }^{446}$ zu rechtfertigen, welche dann formal oder auch empirisch-plausibilisierend zur Begründung von spezifischen Werten genutzt werden können. Die Gründe für die Auswahl der Basiswertungen sind nach KÜPPER wiederum letztlich philosophisch zu fundieren, z. B. durch metaphysische Argumentation, durch das Postulat der Vernunft oder durch konzeptionelle Begründungen, z. B. im Rahmen vertragstheoretischer Überlegungen. ${ }^{447}$ Ein solch konzeptionell strukturiertes Vorgehen könne die Akzeptanz normativer Aussagen deutlich erhöhen. In diesem Sinne postuliert KUPPER: ,An die Stelle der Behauptung, eine bestimmte Norm sei beispielsweise als ethisch gerechtfertigt oder als zweckmäßig anzusehen, tritt ein Begründungszusammenhang, dessen Schritte sich logisch oder empirisch überprüfen lassen und zu einer Norm führen, für die man möglicherweise einen höheren

445 Küpper, H.-U. (2016), S. 17; Küpper, H.-U. (2015), S. 328; Küpper, H.-U. (2011), S. 179; Küpper, H.-U. (2009), S. 789; Küpper, H.-U. / Schreck, P. (2009), S. 28. Vgl. auch Küpper, H.-U. (2007), S. 262.

${ }^{446}$ Eine solche Basiswertung könnte bspw. in einem Weltethos gefunden werden, wie dieses insbesondere in der Tradition von KÜNG erforscht wird. Vgl. Küpper, H.-U. (2016), S. 26; Küpper, H.-U. (2011), S. 213 sowie grundlegend die Überlegungen sowie Ergebnisse in Küng, H. (2016); Küng, H. (2012).

447 Vgl. Küpper, H.-U. (2015), S. 320-321; Küpper, H.-U. (2011), S. 174-176; Küpper, H.U. (2007), S. 260-262. 
Grad an Akzeptanz annehmen kann."448 Diese Überlegungen fasst nochmals die nachfolgende Abbildung 3.13 zusammen.

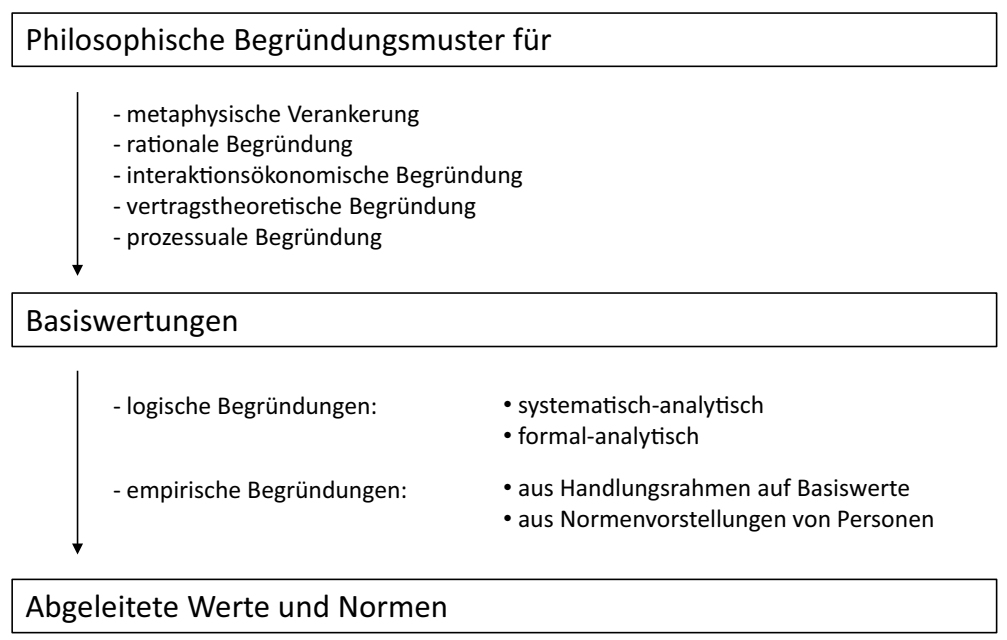

Abbildung 3.13 Begründung von Werten und Normen nach KUPPER ${ }^{449}$

Die Konzeption KÜPPER wird von ihm neben normativen und deskriptiven Ansätzen als dritter konzeptioneller Zugang zu wirtschaftsethischen Fragen erachtet, wobei sie beide Perspektiven konzeptionell innovativ vereine. So sehe die analytische Ethik, ,ihren Gegenstand in der Untersuchung von Wertproblemen und nutzt hierzu sowohl deskriptive als auch normative Aussagen. “450 Dabei geht es ersichtlicherweise nicht um eine Letztklärung normativer Fragen, sondern um eine Unterstützung des Managements in einer transparenteren, konzeptionell unterstützten Durchdringung ethischer Problemstellungen ${ }^{451}$, welches auch für diese Arbeit von besonderer Relevanz ist. Zentral ist in diesem Kontext folglich, ,die in Unternehmungen tätigen und entscheidenden Personen für moralische Probleme in ihrer Tätigkeit zu sensibilisieren und ihnen Instrumente an die Hand

\footnotetext{
448 Küpper, H.-U. (2011), S. 175, in der Quelle fett herv.

${ }^{449}$ Küpper, H.-U. (2016), S. 26; Küpper, H.-U. (2015), S. 321; Küpper, H.-U. (2011), S. 174. Vgl. auch Küpper, H.-U. (2007), S. 261.

${ }^{450}$ Küpper, H.-U. (2011), S. 108.

${ }^{451}$ Vgl. Küpper, H.-U. (2016), S. 25-26; Küpper, H.-U. (2015), S. 331-332; Küpper, H.-U. (2011), S. 164-165; Küpper, H.-U. / Schreck, P. (2009), S. 27; Küpper, H.-U. (2007), S. 265.
} 
zu geben, mit denen sie diese bewältigen können." ${ }^{452}$ Somit sei es letztlich die Aufgabe einer betriebswirtschaftlichen Beschäftigung mit unternehmensethischen Fragestellungen, dem Entscheidungsträger „Wissen zur Verfügung zu stellen, mit dem er die ethische bzw. Wertdimension seiner Entscheidungen bewusster wahrnehmen und hierdurch verantwortlich handeln kann. “453

\subsection{Englischsprachige Beiträge der Business Ethics im Vergleich}

Die vorausgegangenen Ausführungen haben die intensiv geführte und breit gefächerte konzeptionelle Debatte des deutschsprachigen Raumes aufgezeigt, welche zu einer Vielzahl differenziert ausgearbeiteter Wirtschaftsethiken geführt hat. Vergleicht man diesen Forschungsstand mit dem des angelsächsischen Raumes, so zeigen sich einige Unterschiede, welche im Folgenden diskutiert werden, bevor abschließend ein kurzer Überblick über einige wirtschaftsethische Beiträge der Business Ethics gegeben wird.

Im Vergleich zum deutschsprachigen Raum fällt zuerst einmal die bereits relativ frühe Beschäftigung mit wirtschaftsethischen Fragestellungen auf. ${ }^{454}$ In diesem Sinne wurde in der Literatur lange Zeit darauf hingewiesen, dass die deutschsprachige Erforschung wirtschaftsethischer Fragestellungen denen des angelsächsischen Raumes hinterherhinke. So konstatierten STEINMANN und LOHR noch Mitte der 1990er Jahre: „The German debate on business ethics has often been estimated to be at least 10 years behind the state of the art in the United States. “455 Während bei deutschsprachigen Fachvertretern durch den bereits diskutierten historischen Entstehungsprozess der BWL zahlreiche Vorbehalte

\footnotetext{
452 Küpper, H.-U. (2015), S. 326-327.

453 Küpper, H.-U. (2011), S. 165.

454 So finden sich erste systematische Ansätze nach MATTEN und PALAZzo bereits in den 1950er Jahren bei Howard R. Bowen. Vgl. Matten, D. / Palazzo, G. (2008), S. 52 sowie die dort referenzierten Ausführungen in Bowen, H. R. (2013).

455 Steinmann, H. / Löhr, A. (1996), S. 22, in der Quelle ebenfalls herv. In diesem Sinne betont auch VOGEL: ,While interest in this subject was largely confined to the United States during the 1970s, during the 1980s it spread to a number of other capitalist nations as well.“ (Vogel, D. (1992), S. 34). Auch HANSON hebt in diesem Kontext hervor: „Business ethics first appeared as a management concern and as an academic subject in the United States in the late 1970s." (Hanson, K. O. (2018), S. 544). Vgl. auch Bowie, N. E. (2000), S. 7. Vgl. für den spezifisch deutschsprachigen Kontext auch Grabner-Kräuter, S. (2005), S. 141-142.
} 
gegenüber einer Beschäftigung mit normativen Fragestellungen in betriebswirtschaftlichen Konzeptionen existierten, kann im angelsächsischen Bereich eine wesentlich unbefangenere Auseinandersetzung mit dieser Thematik festgestellt werden, welche sich auch in einer bis heute recht unumstrittenen Aufnahme ethischer Kursinhalte in wirtschaftswissenschaftlichen Studiengängen zeigt. So betonen auch KUPPER und SCHRECK: „Während ,Business Ethics“ in vielen Lehrprogrammen und Lehrbüchern anglo-amerikanischer Hochschulen einen festen Platz einnimmt, ist die Verankerung von Unternehmensethik in der deutschen BWL umstritten. “456 Dies zeigt sich auch in der tendenziellen Unterrepräsentation genuin wirtschaftsethischer Lehrstühle im deutschsprachigen Raum, wohingegen angelsächsische Professuren im Bereich der Business Ethics als weitverbreitet und allgemein anerkannt gelten können. In diesem Sinne heben auch STEINMANN und LOHR hervor: „If one compares the research and teaching in the field of Business Ethics with the situation in the United States, then one has to note a clear deficit. Serious arguments and resistance have been to the fore against the establishment of independent university chairs in Business Ethics. “457

Dabei zeigt sich jedoch auch ein konzeptionell im Vergleich zur deutschsprachigen Forschung und Lehre deutlich anderer Zugang zum Themengebiet der Wirtschaftsethik. ${ }^{458}$ Während die deutschsprachige Forschung, wie aufgezeigt, sehr stark auf konsistente, umfangreiche Theoriebildung und -begründung mit der Intention einer Erschaffung widerspruchsfreier (Begriffs-)Systeme fokussiert, ist die angelsächsische Tradition dominant durch eine Praxisorientierung charakterisiert, mit dem Ziel der Lösung praktischer Managementprobleme. So steht weniger die Entwicklung umfassender Konzepte im Vordergrund als vielmehr die empirische Sammlung von Evidenzen und die Entwicklung von Tools ${ }^{459}$, welche Managern in der beruflichen Praxis hilfreich sein können, d. h. für praktische

\footnotetext{
456 Küpper, H.-U. / Schreck, P. (2009), S. 14. In diesem Sinne konstatierten bereits STEINMANN und LOHR: „One should, however, note that ethical aspects have had hardly any attention in economics teaching in the Federal Republic." (Steinmann, H. / Löhr, A. (1992), S. 140). Vgl. ähnlich auch die Ausführungen bei STEINMANN und OPPENRIEDER: „Im Vergleich zu diesen ,forcierten“ Anstrengungen in den USA nehmen sich die europäischen Bemühungen um die Konstituierung einer Lehre von der Unternehmensethik eher bescheiden aus.“ (Steinmann, H. / Oppenrieder, B. (1985), S. 170).

457 Steinmann, H. / Löhr, A. (1992), S. 139-140, in der Quelle ebenfalls herv. Vgl. auch Matten, D. / Palazzo, G. (2008), S. 60; Enderle, G. (1996), S. 43.

${ }^{458}$ Für eine ausführliche konzeptionelle Diskussion, welche auch eine Berücksichtigung französischsprachiger Beiträge enthält, vgl. die Ausführungen in Seidel, F. / Schlierer, H.-J. / Tovey, I. (1998).

${ }^{459}$ PALAZZO bezeichnet in diesem Kontext prägnant den ,,angelsächsische[n] intellektuelle[n] Stil“ als „Turning Theories into Tools“ (Palazzo, B. (2005), S. 184).
} 
Probleme viable Lösungen ${ }^{460}$ offerieren, ohne Anspruch auf strenge Theoriekonsistenz oder eine Geschlossenheit zugrunde liegender theoretischer Systeme zu erheben.

Die Gründe für die unterschiedliche Ausrichtung beider wirtschaftsethischer Traditionen sind dabei intensiv in der Literatur, insbesondere in den 1990er und zu Beginn der 2000er Jahre, diskutiert worden. Aus theoriegeschichtlicher Perspektive kann zum einen ein differierendes philosophisches Fundament zur Begründung der unterschiedlichen Entwicklung herangezogen werden. So argumentiert beispielsweise PALAZZO, dass die angelsächsische Forschung „durch den starken Einfluss des Pragmatismus ${ }^{461}$ als der dominanten erkenntnistheoretischen und philosophischen Tradition ${ }^{\text {“462 }}$ beeinflusst sei. Das Konzept des Pragmatismus, welches insbesondere durch die Arbeiten von PEIRCE ${ }^{463}$ begründet wurde ${ }^{464}$, postuliert hierbei ,einen Vorrang von Praxis vor rein theoretischen Überlegungen“465. Spezifisch gilt z. B. folglich der Standpunkt, dass ,die Wahrheit einer Erkenntnis nichts anderes bedeutet, als dass sie auf lange Sicht von einer Gemeinschaft der Forschenden vertreten werden kann. Eine unbezweifelbare Gewissheitsbasis wird nicht zugelassen bzw. als nicht notwendig erachtet. “466 In diesem Sinne subsumiert auch CAVANAGH, dass es die zentrale Idee des Pragmatismus sei, zu klären, „how to get the job done without much

${ }^{460}$ Der Begriff der Viabilität, welcher ursprünglich aus der Biologie stammt, wurde von ERNST VON GLASERSFELD in den breiteren wissenschaftlichen Diskurs eingeführt und gegen den klassischen Wahrheitsanspruch wissenschaftlicher Erkenntnis positioniert. In diesem Sinne gilt: „Begriffe, Theorien und kognitive Strukturen im allgemeinen sind viabel bzw. überleben, solange sie die Zwecke erfüllen, denen sie dienen [...].“ (Glasersfeld, E. v. (1992), S. 141). Vgl. einführend auch Köck, W. K. (2015), S. 369-373.

${ }^{461} \mathrm{Vgl}$. hierzu paradigmatisch die Ausführungen bei Rosenthal, S. B. / Buchholz, R. A. (2000); Michalos, A. C. (1995).

462 Palazzo, B. (2005), S. 185. Vgl. hierzu auch Enderle, G. (1996), S. 42; Steinmann, H. / Löhr, A. (1992), S. 139. In diesem Sinne hebt auch PALAZZO pointiert die ,different, habits of the heart" underlying German and American business ethics" (Palazzo, B. (2002), S. 195) hervor.

${ }^{463}$ Vgl. einführend Peirce, C. S. (1973). Zentrale klassische Beiträge zum Pragmatismus finden sich auch bei JAMES. Vgl. hierzu James, W. (1995).

${ }^{464}$ Vgl. Fetzer, J. (2004), S. 46. In der Literatur wird PEIRCE daher häufig auch als ,,Vater des Pragmatismus“ (Viola, T. (2018), S. 2) bezeichnet. Vgl. einführend auch Lenk, H. (1975).

465 Fetzer, J. (2004), S. 46.

${ }^{466}$ Fetzer, J. (2004), S. 46. 
concern for inconsistencies. Simply put, pragmatism says that which works is by that fact good and true. “467

Dagegen zeigt die deutschsprachige Tradition eine starke Prägung durch den Deutschen Idealismus, welcher die konzeptionell strenge „Entwicklung von Theorien und Prinzipien in den Vordergrund" ${ }^{\text {“468 }}$ rückt. Dies zeigen auch die großen Systementwürfe der klassischen Autoren wie KANT, FICHTE, SCHELLING und HEGEL mit ihrer intensiven Suche nach umfassenden, geschlossenen und in sich konsistenten philosophischen Begriffssystemen. ${ }^{469}$ Folglich herrscht aus der Perspektive der deutschsprachigen Wirtschaftsethikforschung häufig eine recht deutliche Skepsis bis hin zu Unverständnis gegenüber der stark praktisch bzw. anwendungsorientierten angelsächsischen „Business Ethics“ vor, welcher z. B. oftmals konzeptionelle „Profillosigkeit“470 oder eine schwache theoretische Fundierung vorgeworfen wird. ${ }^{471}$ In Abgrenzung hierzu gelte dagegen ,that Continental Europeans, though in varying degrees, tend to focus first on theoretical issues before they address, if ever, the practical challenges." ${ }^{\text {*47 }}$ In diesem Sinne betonen auch STEINMANN und LOHR die Tendenz der deutschsprachigen Forschung, sich in der „Diskussion um Unternehmensethik immer wieder in theorieträchtigen Grundsatzprogrammen“473 zu verfangen. Darauf aufbauend wird vielfach auch ein differierendes Tätigkeitsverständnis der deutschsprachigen und angelsächsischen Wirtschaftsethiker postuliert. So betonen auch SEIDEL,

467 Cavanagh, G. F. (1976), S. 172. Vgl. ähnlich auch die Ausführungen der neuesten Auflage zum Pragmatismus in Cavanagh, G. F. (2010), S. 339.

468 Palazzo, B. (2005), S. 186.

${ }^{469}$ Ganz in diesem Geiste betont auch STADLER: „Begriffsklarheit ist die Voraussetzung ernster Forschung; die Schaffung einwandfreier Begriffe und ihre Vereinigung zu einem geschlossenen System, erste und grundlegende Aufgabe jeder Wissenschaft." (Stadler, M. (1932), S. 5).

${ }^{470}$ Palazzo, B. (2005), S. 185.

${ }^{471}$ In diesem Sinne subsumieren auch STEINMANN und LOHR, dass klassische Disziplinen „like philosophy and economics, often disqualified the international (and mainly U.S.) debate as theoretically poor and merely instrumental.“ (Steinmann, H. / Löhr, A. (1996), S. 23). Ähnlich argumentiert auch PALAZZO, welche die teils ambiguitive Evaluation der pragmatischen Forschungstradition aus deutschsprachiger Perspektive noch stärker hervorhebt: ,Seen from a distance, the American hands-on activities impress German theorists in business ethics and fill them with a peculiar mixture of feelings: amazement, envy and disapproval." (Palazzo, B. (2002), S. 197). Vgl. hierzu auch Grabner-Kräuter, S. (2005), S. 152-154.

472 Enderle, G. (1996), S. 42.

473 Steinmann, H. / Löhr, A. (1995b), S. 238. Auch WIELAND hebt im deutschsprachigen Raum analog das lange Zeit ,abstrakte[] Niveau“ (Wieland, J. (1993), S. 13) des wirtschaftsethischen Diskurses hervor. 
SCHLIERER und TOVEY: „The pragmatic qualities of the American approach effectively owe as much to the management style and objectives of most American business schools as the highly theoretical approach of the Germans does to the academic and scientific (wissenschaftlich) demands intrinsic to Betriebswirtschaftslehre as it is fostered in German Universities. “474

Während im deutschsprachigen Raum folglich stark die Forschung aus originären Erkenntnisgründen betrieben wird, steht im angelsächsischen Raum wesentlich stärker die didaktische Tätigkeit der Ausbildung zukünftiger Manager im Fokus, welche die Auseinandersetzung mit normativen Fragestellungen im Sinne von ,good business" als Mittel zur erfolgreichen Unternehmensführung auffasst. Dies äußert sich zum einen in dem starken Fokus auf Case Studies, in welchen konkrete Einzelfälle ethischer Probleme aus der Managementpraxis diskutiert werden. So betonen auch FRENCH und GRANROSE die Bedeutung des Case Study-Ansatzes zur Vermittlung wirtschaftsethischer Inhalte und heben hervor: "We believe that the case study approach has proven itself to be the most stimulating and productive method of helping both students and business people develop the skills necessary to cope effectively with the moral or ethical dilemmas that arise in the competitive business world. And we believe that actual cases, rat-

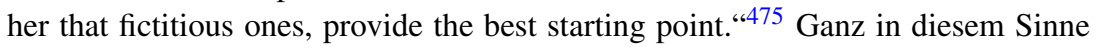
fokussieren auch GOODPASTER, NASH und DE BETTIGNIES in ihrem Einführungswerk auf konkrete Praxisbeispiele und betonen ,teaching and learning ethics by the case method as an activity which, when undertaken with certain epistemological, analytical, and curricular convictions, provides a powerful approach to professional education. “476

Der Fokus auf die Ausbildung künftiger Manager spiegelt sich wiederum in dem ebenfalls stark ausgeprägten Fokus auf die wirtschaftsethische Mikroebene des handelnden Individuums als zentraler Entscheidungsinstanz wider. Während, wie gezeigt wurde, neben führungs- bzw. individualethischen Überlegungen in den deutschsprachigen Konzeptionen auch intensive Bemühungen zur Konzeption von makro- bzw. mesoethischen Ansätzen aufzufinden sind, weisen die zentralen angelsächsischen Werke häufig einen starken Mikrofokus auf. So konstatiert auch ENDERLE: „One of their major tasks consists in educating future managers, which

\footnotetext{
${ }^{474}$ Seidel, F. / Schlierer, H.-J. / Tovey, I. (1998), S. 237-238, in der Quelle ebenfalls herv. ${ }^{475}$ French, W. A. / Granrose, J. (1995), S. 3.

${ }^{476}$ Goodpaster, K. E. / Nash, L. L. / De Bettignies, H.-C. (2006), S. 14. Dabei sei auch ,the case method as a form of pedagogy particularly suited to the subject matter of business ethics." (S. 3, in der Quelle ebenfalls herv.). Vgl. auch die einführenden Überlegungen bei Ladenson, R. F. (2009).
} 
is why they mainly deal with micro-issues and, to a lesser extent, meso-issues. “477 Ähnlich hebt auch VoGEL für den spezifisch US-amerikanischen Kontext hervor: „Americans tend to emphasize the role of the individual as the most critical source of ethical values, while in other capitalist nations relatively more emphasis is placed on the corporation as the locus of ethical guidance. ${ }^{4478}$

Diese recht unterschiedliche methodische Vorgehensweise schlägt sich auch in den vorherrschenden Kompendien der angelsächsischen Wirtschaftsethik wieder. So sind die meisten der klassischen Lehrbücher der Business Ethics erkennbarerweise klar auf die Diskussion einzelner Fallbeispiele fokussiert und weisen meist nur eine geringe spezifisch-konzeptionelle Fundierung auf. ${ }^{479}$ Als wenige Ausnahmen einer expliziten konzeptionellen Positionierung können hier z. B. die Werke von BowIE oder SISON genannt werden, wobei BowIE in seinen Ausführungen auf die Pflichtenethik KANTS rekurriert ${ }^{480}$, während SISON seine wirtschaftsethischen Überlegungen auf der aristotelischen Tugendethik ${ }^{481}$ aufbaut. ${ }^{482}$ Dennoch kann letztlich auch bei diesen Wirtschaftsethikern konstatiert werden, dass diese vor allem einen Transfer klassischer ethischer Paradigmen auf praktische Fälle vornehmen und dabei keine umfassende eigenständige Theorieentwicklung betreiben. ${ }^{483}$ Aus der vielfach praktisch-instrumentellen Ausrichtung kann schließlich auch ein Fokus auf die Entwicklung von „Ethiktools“, welche eine konzeptionell fundierte Entscheidungsunterstützung normativer Fragen intendieren, aufgefunden werden. Ein bekanntes Beispiel stammt hierbei von

${ }^{477}$ Enderle, G. (1996), S. 44. Vgl. hierzu auch Matten, D. / Palazzo, G. (2008), S. 51.

478 Vogel, D. (1992), S. 44. Vgl. ähnlich auch Vogel, D. (1993), S. 169.

${ }^{479}$ Vgl. hierzu z. B. die Ausführungen in Crane, A. et al. (2019); Ferrell, O. C. / Fraedrich, J. / Ferrell, L. (2017); Shaw, W. H. (2017); Stanwick, P. A. / Stanwick, S. D. (2016); Velasquez, M. G. (2014); Weiss, J. W. (2014); De George, R. T. (2010); Goodpaster, K. E. / Nash, L. L. / De Bettignies, H.-C. (2006); French, W. A. / Granrose, J. (1995).

${ }^{480}$ Vgl. Bowie, N. E. (2017); Bowie, N. E. (2013); Bowie, N. E. (2000); Bowie, N. E. (1998a); Bowie, N. E. (1998b). Vgl. auch die Ausführungen bei Freeman, R. E. (2012), welcher BowIES Ansatz als pragmatische Interpretation der Lehre KANTS deutet.

${ }^{481}$ Vgl. Sison, A. J. G. / Ferrero, I. / Guitián, G. (2019); Sison, A. J. G. (2015); Sison, A. J. G. / Fontrodona, J. (2012); Sison, A. J. G. (2011a); Sison, A. J. G. (2011b); Sison, A. J. G. (2008). Ein weiterer bekannter Vertreter einer tugendethischen Wirtschaftsethik ist Solomon. Vgl. hierzu die Ausführungen in Solomon, R. C. / Martin, C. (2004); Solomon, R. C. (1999); Solomon, R. C. (1992a).

${ }^{482}$ Vgl. z. B. Neuhäuser, C. (2011c), S. 164, welcher neben diesen zwei klassischen Strömungen auch die Beiträge von RAWLS als dritte bedeutsame Theorietradition auffasst.

${ }^{483}$ In diesem Sinne konstatiert auch BowIE: ,,[.] I am simply using Kantianism as a theoretical normative framework for telling a story about what a Kantian vision of capitalist business organizations would be like.“ (Bowie, N. E. (1998a), S. 38). 
FISHER und LOVELL, welche ein Framework entwickeln, das die verschiedenen konzeptionellen Perspektiven (z. B. Tugend- und Pflichtenethik, aber auch das persönliche Gewissen) in ein integratives Framework einbindet, so dass hierauf aufbauend eine ausgewogene, transparente normative Argumentation erleichtert wird. ${ }^{484}$ Dies wiederum korrespondiert mit dem aufgezeigten starken Fokus der „Business Schools“ auf eine angewandte Managementlehre, welche eine praxisorientierte Ausbildung anstelle einer eher akademischen Betrachtung wirtschaftsethischer Zusammenhänge intendieren.

Die vorausgegangenen Überlegungen haben nach Erörterung der zentralen terminologischen Grundlagen der Ethik die im deutschsprachigen Raum intensiv geführte Diskussion um die konzeptionelle Ausrichtung der Wirtschaftsethik aufgezeigt, welche eine Vielzahl konkurrierender Konzeptionen hervorbrachte. In diesem Zusammenhang wurde auch deutlich, dass konträr hierzu die angelsächsische Wirtschaftsethiktradition ein wesentlich stärker praktisch orientiertes Forschungsprogramm verfolgt und daher kaum eigenständige wirtschaftsethische Ansätze erarbeitet hat, welche wiederum in konzeptionellen Diskursen umfassend kritisierbar wären. ${ }^{485} \mathrm{Im}$ Nachfolgenden werden daher insbesondere die deutschsprachigen Konzeptionen einer kritischen Würdigung unterzogen. Dabei zeigt sich neben der umfangreichen Kontroverse um die „richtige“ Ausrichtung der Wirtschaftsethik, auch in der Zusammenschau mit den pragmatischen angelsächsischen Überlegungen, die Notwendigkeit einer Neukonzipierung von Unternehmensethik, welche als originäre Mesoethik überzeugend eine praktische Relevanz und theoretische Fundiertheit verbindet.

\footnotetext{
484 Vgl. Fisher, C. / Lovell, A. (2009), S. 139-140. In der neuesten Auflage wurden die detaillierten Ausführungen deutlich gekürzt und es findet sich ein Internetlink, welcher zu einer interaktiven Applikation des nochmals überarbeiteten Frameworks führt. Vgl. hierzu Fisher, C. / Lovell, A. (2013), S. 142-143. Vgl. in diesem Kontext auch die Frameworks von Jiambalvo, J. (2020), S. 19; Scharding, T. (2018), S. 54-56; Stuart, I. / Stuart, B. / Pedersen, L. J. T. (2014), S. 46; Gampel, E. H. (2009), S. 55-64.

${ }^{485}$ Eine Ausnahme bildet hier die Stakeholder-Theory, welche vielfach auch zum Gegenstandsbereich der Business Ethics gerechnet wird. Vgl. z. B. Crane, A. et al. (2019), S. 59-65; Ferrell, O. C. / Fraedrich, J. / Ferrell, L. (2017), S. 30-52; Fisher, C. / Lovell, A. (2013), S. 348-357 sowie konzeptionell auch Grabner-Kräuter, S. (2005), S. 154-157. Vgl. auch die Überlegungen bei Weiss, J. W. (2014), welcher diese explizit mit dem Stakeholderkonzept fundiert.
} 


\subsection{Kritische Würdigung des Forschungsstandes}

Wie die vorausgegangenen Ausführungen zeigten, hat sich insbesondere die deutschsprachige Forschung auf die Erarbeitung detaillierter Konzeptionen fokussiert. Im Weiteren werden nun neben den konzeptionellen Verdiensten auch die potentiellen Limitationen ${ }^{486}$ der zuvor aufgezeigten Schulen der Wirtschaftsethik problematisiert sowie auch die kritische Auseinandersetzung zwischen den einzelnen Theorietraditionen aufgezeigt.

\subsubsection{Reflexion der klassischen Ansätze des deutschsprachigen Raums}

Die klassische diskursethische Konzeption von STEINMANN und LÖHR kann in vielerlei Hinsicht als wegweisend erachtet werden. Die Autoren betonen zum einen die zentrale Notwendigkeit, sich als Unternehmen aktiv um die gesellschaftliche Legitimität betrieblicher Handlungen zu bemühen. Legales Handeln kann dabei als nicht hinreichend zum Erhalt der gesellschaftlichen Legitimität erachtet werden. In diesem Sinne gilt richtigerweise: „Compliance is not enough“! 487 Darüber hinaus wenden sich STEINMANN und LÖHR aber auch explizit gegen manipulative oder rein monologische Legitimierungsversuche und betonen die Notwendigkeit eines integrativen Diskurses als zentrales konzeptionelles Fundament ihrer Ethik. Dabei gilt beispielsweise, dass hinsichtlich des zu führenden Diskurses potentiell alle Betroffenen Zugang besitzen müssen und letztlich das bessere Argument ${ }^{488}$ zählt.

${ }^{486}$ Eine umfassende Diskussion der zentralen Wirtschaftsethiken des deutschsprachigen Raums findet sich auch bei Küpper, H.-U. (2011), S. 130-164.

${ }^{487}$ Lingnau, V. / Fuchs, F. (2021), S. 11; Kennedy-Glans, D. / Schulz, R. (2005), S. XIX; Wheeler, W. A. (1992), S. 10. Diese Auffassung wird sogar in der Schule HomanNs geteilt, so heben beispielsweise LUTGE und UHL hervor, dass Compliance lediglich ,als ethische Minimalanforderung “ (Lütge, C. / Uhl, M. (2017), S. 171) aufzufassen wäre. Dabei erscheint jedoch grundsätzlich unproblematisiert, dass (gerade in illiberalen Regierungssystemen) auch illegitime rechtliche Regelungen existieren können, so dass die Legalität nicht vollständig innerhalb der Sphäre der Legitimität verortet sein muss bzw. keine notwendige Teilmenge hiervon darstellt. Vgl. hierzu auch die konzeptionellen Überlegungen in Abschnitt 2.5.1.

${ }^{488}$ Hierbei sind, wie bereits in den Grundlagen aufgezeigt, die Existenz guter bzw. persuasiver Gründe zentral. Gute Gründe liegen nach STEINMANN und LÖHR , dann vor, wenn über sie ein allgemeiner und freier Konsens aller Betroffenen über ihre Berechtigung erzielt worden ist.“ (Steinmann, H. / Löhr, A. (1997), S. 10). 
Die Konzeption von STEINMANN und LÖHR wurde jedoch auch einer umfassenden kritischen Würdigung unterzogen. Denn zuerst einmal ist fraglich, ob stets alle Betroffenen faktisch in einem Diskurs partizipieren können. Insofern bleibt der herrschaftsfreie Diskurs, analog zur Kritik an der zugrunde liegenden Konzeption von HABERMAS, stets ein Ideal, welches es praktisch zu approximieren gilt. ${ }^{489}$ Neben der diskursiven Ausrichtung der Konzeption wurde jedoch auch die generelle Methodik als ,angewandte Ethik ${ }^{\text {“490 }}$, insbesondere von den zwei konkurrierenden Schulen um HOMANN sowie ULRICH, kritisch rezipiert. ${ }^{491}$ So verbleibe die Konzeption von STEINMANN und LÖHR aus der Perspektive HomANns sowie seiner Schüler auf der reinen Ebene des Appellierens und vernachlässige damit den Wettbewerbsdruck, welchem sich Unternehmen ausgesetzt sähen. Als Kernkritikpunkt wird in diesem Sinne in der Literatur meist geschlussfolgert, dass hier die Ethik ,gegen die Ökonomie in Stellung gebracht [werde]“, welches letztlich „auf Kosten der Wirtschaftlichkeit [gehe].“492 Auch aus der Schule um UlRICH verharrt die Konzeption von STEINMANN und LÖHR lediglich auf der Stufe eines Korrektivs bzw. einer Reparaturethik, ohne das zugrunde liegende Problem bzw. die Ursachen fragwürdiger Handlungen näher zu erörtern („Reflexionsstopp“493). Auf diese Vorwürfe haben wiederum STEINMANN und LOHR in einer Vielzahl von Publikationen reagiert, in welchen sie ihre Position verteidigen und beispielsweise hervorheben, dass auch, aber nicht nur (oder primär) die Rahmenordnung als Ort der Moral aufzufassen wäre. ${ }^{494}$ Abschließend kann damit festgehalten werden, dass in der Konzeption von STEINMANN und

${ }^{489}$ Dies betonen auch STEINMANN und LOHR. Sie weisen darauf hin, dass Ideale als normative Leitlinien trotzdem von konzeptioneller Relevanz seien, an welchen letztlich die Realität gemessen werden könne. Vgl. Steinmann, H. / Löhr, A. (1994a), S. 80-81.

${ }^{490}$ Dabei ist aus fachwissenschaftlicher Sicht die Kritik als ,,angewandte Ethik“ allerdings irreführend, da, wie aufgezeigt wurde, die gesamte wirtschaftsethische Forschung als angewandte Disziplin aufgefasst werden kann. Vgl. hierzu nochmals Abschnitt 3.2.3.

${ }^{491}$ Vgl. im Überblick Göbel, E. (2020), S. 83-84.

492 Göbel, E. (2020), S. 83.

493 Ulrich, P. (2017), S. 14; Ulrich, P. (2016a), S. 15. Vgl. auch Ulrich, P. (2004b), S. 17.

${ }^{494}$ So heben STEINMANN und LOHR hervor: „Die Kritik an unserer Position entsteht hier also erst aus der unzulässigen definitorischen Unterstellung, wir würden den ,Ort der Moral ' nur auf der Unternehmensebene festmachen, die Regeln der Rahmenordnung dagegen nicht als Quelle moralisch gerechtfertigter Orientierungen ansehen. Dabei haben wir doch immer und unmißverständlich darauf hingewiesen, daß letztere zum Regelungsbereich der Wirtschaftsethik gehören und insofern - bei guter Begründung - als legitime Ansprüche an die Unternehmen gerichtet werden!“ (Steinmann, H. / Löhr, A. (1994a), S. 134, in der Quelle fett herv.). 
LÖHR der Fokus auf die Grenzen des reinen ökonomischen Prinzips (unter vornehmlich legalistischer Argumentation) aus legitimatorischer Perspektive sowie die Hervorhebung der Relevanz eines diskursethischen Fundaments bedeutsam erscheinen. Dabei stellt jedoch, unbeschadet der Frage, welcher Betriebszweck nun als unternehmenskonstitutiv erachtet wird, der Fokus auf den legitimen Gewinn eine zu problematisierende Verengung dar, lässt dieser doch wesentliche Ansprüche, z. B. hinsichtlich des Umgangs mit Personal und Zulieferern, welche auch außerökonomische Größen umfassen, außen vor. In diesem Sinne zeigt die Konzeption zwar eine intensive Auseinandersetzung mit einer kritischen Reflexion des unternehmerischen Gewinnstrebens. Darüber hinaus erscheint es jedoch sinnvoll, dass eine Mesoethik wesentlich breiter und direkt mit einer kritischen Reflexionsmöglichkeit aller Stakeholderansprüche beginnt und diese einer Legitimitätsprüfung unterzieht. Zudem weist die Konzeption in der Tat einen durchaus appellativ geprägten Charakter auf. So betont auch STEINMANN explizit: ,,[C]orporate ethics should be understood [...] as a moral duty [...]. "495 In diesem Zusammenhang bleibt jedoch die Frage offen, wie die Notwendigkeit von Unternehmensethik überzeugend zu begründen wäre, ohne lediglich normativ besseres Handeln einzufordern, sondern auch die praktische Notwendigkeit für die Unternehmensführung persuasiv zu begründen. Dieser Frage wird sich die Arbeit im weiteren Verlauf dezidiert widmen.

Einen anderen konzeptionellen Zugang wählen wiederum HOMANN und seine Schüler, welche vor allem die ordnungspolitische Ebene als zentrales, wirtschaftsethisch wirksames Regelset auffassen. Hierbei gehe es gerade ,nicht um eine Ersetzung der Individual-, Handlungs- oder Tugendmoral [.], sondern um deren Stützung [...]." ${ }^{496}$ Zentrale Überlegung der Konzeption ist folglich der Schutz vor Ausbeutung durch für alle Akteure gleichsam wirksame, verbindliche Standards in Dilemmasituationen. HOMANN hebt dabei richtigerweise die potentielle Ausbeutbarkeit von Moral hervor, welche durch die Instabilität bzw. mögliche Asymmetrie einer freiwilligen, moralisch motivierten Kontribution resultiert. So reicht möglicherweise bereits die potentielle Defektion eines einzelnen Akteurs, um alle anderen Akteure aus Eigenschutz zu einer präventiven Gegendefektion zu zwingen ${ }^{497}$, wodurch eine institutionelle Absicherung gegen Opportunismus

495 Steinmann, H. (2008b), S. 136. Ähnlich betont auch an anderer Stelle STEINMANN die Übernahme von Verantwortung als ,moralische Pflicht“ (Steinmann, H. (2008a), S. 339, in der Quelle ebenfalls herv.).

496 Homann, K. (2014), S. 64.

497 Vgl. hierzu nochmals Homann, K. (2015), S. 43; Homann, K. / Lütge, C. (2013), S. 27; Homann, K. / Suchanek, A. (2005), S. 36; Homann, K. (2002f), S. 14; Homann, K. (1990), S. 35 . 
zentral erscheint. ${ }^{498}$ Diese wird jedoch dann vornehmlich auf der makroethischen Ebene der Wirtschaftsordnung verortet.

Die Konzeption Homanns weist neben diesen Erkenntnisgewinnen einige kritikwürdige Annahmen auf und ist in der Literatur besonders aufgrund ihrer ökonomischen Argumentationsstruktur durchaus kritisch rezipiert worden. Neben dem klassischen Kritikpunkt, dass HOMANN moralisch motivierte Handlungen anhand des Motivs „Eigennutz“ zu implementieren intendiert ${ }^{499}$, wurde auch die generelle Steuerungsproblematik über die Rahmenordnung einer kritischen Analyse unterzogen. Insbesondere wird dabei vielfach auf die Steuerungsgrenzen des Rechts als Implementationssphäre moralischer Normen zurückgegriffen. ${ }^{500}$ So betont ebenso GÖBEL die Grenzen einer Verschärfung von Gesetzen, denn Gesetze würden teilweise ignoriert ${ }^{501}$ (unvollständige Kontrollmöglichkeiten), Gesetze enthielten immer auch Grauzonen und bedürften der Auslegung ${ }^{502}$, die Gesetzgebung sei stets lückenhaft ${ }^{503}$ und die Globalisierung ermögliche ein Ausweichen einzelstaatlicher Regelungen. Des Weiteren sei die Wirtschaft vielfach an der Gestaltung der Rahmenordnung mitbeteiligt, so dass ein Regulierungsergebnis hinsichtlich seiner gesamtgesellschaftlichen Legitimität zu hinterfragen sei und zudem bestehe abschließend auch stets die potentielle Gefahr einer

${ }^{498}$ Vgl. Homann, K. (2002f), S. 18: „Ohne Sicherung durch i.w.S. ,ökonomische“ Vorteile kann die Moral keinen Bestand haben.“

${ }^{499}$ So betont auch GÖBEL: ,Die einseitige und unbändige Verfolgung ihrer eigenen Interessen reicht nicht aus, um das Gemeinwohl zu fördern, auch nicht im Rahmen einer regulierten Marktwirtschaft." (Göbel, E. (2017), S. 24). In diesem Sinne betonen auch STEINMANN und LOHR, dass ,schon A. Smith richtigerweise darauf hingewiesen [hat], daß jede Marktwirtschaft nur auf der Grundlage gemeinsam geteilter Moralvorstellungen (Vertragstreue, Zahlungsmoral, Respekt für den Marktpartner etc.) funktionsfähig ist.“ (Steinmann, H. I Löhr, A. (1994a), S. 27).

${ }^{500}$ Vgl. hierzu auch klassisch Stone, C. D. (1975).

501 So ist auch evident, dass eine praktizierte, ernsthafte Beachtung von Rechtsnormen neben ihrer formalen Existenz zweifellos der Moral einer Gesellschaft zufällt. So konstatiert auch HORAZ in seinem berühmten Zitat: „Quid leges sine moribus vanae proficiunt [...]?“ (Horaz (2002), lib. 3, 24, 35-36).

502 Ein klassisches Beispiel wäre hier z. B. auch die Auslegung von „Treu und Glauben“ oder dem ,sittenwidrigen Rechtsgeschäft“ im Rahmen des Bürgerlichen Gesetzbuches. Vgl. hierzu $\S 138$ Abs. 1, 242 BGB (2021).

503 Ähnliche Überlegungen finden sich bereits in den 1990er Jahren bei PIES und BLOMEDREES, welche feststellten, ,daß die marktwirtschaftlichen Rahmenordnungen, die notwendig lückenhaft sind, in modernen Gesellschaften immer größere Defizite aufweisen und daher ihre moralische Entlastungsfunktion für unternehmerisches Handeln immer weniger erfüllen.“ (Pies, I. / Blome-Drees, F. (1993), S. 752). 
Regelungswut $^{504}$, die der freien Marktwirtschaft wiederum entgegenstehe. ${ }^{505}$ Etliche weitere Autoren haben in diesem Kontext ähnliche Argumente gegen die Konzeption HOMANNS hervorgebracht. So kritisiert beispielsweise auch FENNER die Unterschätzung unternehmerischer Handlungsspielräume, den zeitlichen Verzug der Gesetzgebung, wodurch die Gesetze möglicherweise den aktuellen Legitimitätsvorstellungen einer Gesellschaft hinterherhinkten sowie die Grenzen einzelstaatlicher Regulierung im Rahmen der Globalisierung. ${ }^{506}$ Auch die Schule um STEINMANN folgt der oben ausgeführten Kritik, stellt hierbei aber nochmals verstärkt die Problematik der vielfachen Abstraktheit von Gesetzen heraus und problematisiert die Grenzen der Steuerung durch Rechtsnormen aufgrund faktischer Vollzugsdefizite. ${ }^{507}$ Die aufgezeigten Kritikpunkte potenzieren sich dabei im Rahmen der zunehmenden Globalisierung unternehmerischer Wertschöpfungsketten, für welche kein einheitlicher, global legitimierter Gesetzgeber existiert. Global wirtschaftende Unternehmen können sich so einzelstaatlichen Kontrollen vielfach entziehen. In diesem Sinne betont STEINMANN: „Diese Frage wird mit der Globalisierung immer dringender; denn auf der übernationalen Ebene fehlt ja, wie angedeutet, ein Weltgesetzgeber, der die gemeinsamen Spielregeln

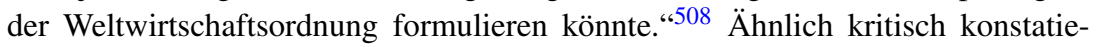
ren auch WETTSTEIN und WADDOCK: „The ongoing economic globalization, however, has rendered this state-centered view increasingly inadequate. [...] $[\mathrm{P}]$ owerful transnational corporations must bear more and more direct responsibility for the impact of their actions [...]." ${ }^{\text {509 }}$

${ }^{504}$ Vgl. hierzu auch die Ausführungen bei Heidenreich, F. (2012), S. 72-73.

505 Vgl. Göbel, E. (2017), S. 21-24.

506 Vgl. Fenner, D. (2010), S. 397-398.

${ }^{507}$ Vgl. Steinmann, H. / Löhr, A. (1997), S. 31; Steinmann, H. / Kustermann, B. (1996), S. 13; Steinmann, H. / Löhr, A. (1994a), S. 115-116; Steinmann, H. / Oppenrieder, B. (1985), S. 178.

508 Steinmann, H. (2004), S. 109, in der Quelle ebenfalls herv. Vgl. hierzu ähnlich auch Steinmann, H. (2011), S. 100 sowie die Ausführungen bei Steinmann, H. (2008b), welcher darauf hinweist, dass ,the law itself is often (and more and more so) unable to settle [conflicts] satisfactorily in the public interest. This is already true for the nation state but is still more relevant today for the global economy, and this for many reasons, one being that there is no world wide political institution which can legitimately and effectively decide upon and enforce global rules (world law giver)." (S. 133-134).

${ }^{509}$ Wettstein, F. / Waddock, S. (2005), S. 304. Auch dieser Kritikpunkt wurde interessanterweise wiederum ebenfalls von den Schülern HOMANNS thematisiert. So betonen auch PIES, BECKMANN und HIELSCHER: ,Nowadays, however, there are definitely global problems, but there is no global government.“ (Pies, I. / Beckmann, M. / Hielscher, S. (2010), S. 274). 
Die aufgezeigte Problematik eines rechtlichen time-lag gilt damit nicht nur für die Verabschiedung einzelner Gesetze, sondern vielmehr auch für die Entwicklung eines globalen wirtschaftspolitischen Systems in noch fundamentalerem Maße, worauf z. B. BESCHORNER hinweist: „Für wirtschaftsethische Fragestellungen relevant ist dabei, dass das für die Steuerung der politischen Rahmenordnung wichtige Rechtssystem zeitlich hinter den Entwicklungen der anderen Teilsysteme hinterher hinkt. “510 Hierdurch kann zumindest faktisch eine primäre Regulierung über eine Wirtschaftsrahmenordnung heute als wenig aussichtsreich eingestuft werden, vielmehr erscheint damit die Konzeption HoMANNS auch wiederum eher als Idealtheorie, welche die Möglichkeit einer hinreichenden Regulierbarkeit auf globaler Ebene voraussetzen muss, will sie denn faktisch über die Rahmensetzung ihre Wirkmächtigkeit auf das unternehmerische Handeln entfalten. Zudem wäre zu bezweifeln, ob die reine Einhaltung von Gesetzen bereits eine hinreichende unternehmerische Legitimitätswirkung entfaltet. So wiesen bereits PIES und BLOME-DrEes in den 1990er Jahren darauf hin, ,daß Legalität und Legitimität zunehmend auseinanderfallen: Gewinnorientiertes Handeln kann heute seine moralische Rechtfertigung immer weniger allein dadurch sicherstellen, daß es die bestehenden Gesetze und geltenden Wettbewerbsregeln beachtet. Die im klassischen Konzept an die Rahmenordnung delegierte Legitimation wirtschaftlichen Handelns fällt unter solchen Bedingungen an die Unternehmen zurück." Betrachtet man das Werk HomanNs, so fällt auf, dass dieser das Themenfeld der Unternehmensethik nur meist relativ kurz und subsidiär ausführt. Hieran anknüpfend subsumiert auch STEINMANN: „Für Unternehmensethik (im hier gemeinten Sinne) wäre kein Platz, jedenfalls kein systematischer Platz.“512 Damit könnte letztlich, wie LÖHR hervorhebt, eine Unternehmensethik „nur die Funktion eines empirisch mehr oder weniger bedeutsamen Lückenbüßers oder eines Anstoßes

Auch SUCHANEK stellt fest: „Die Globalisierung stellt Unternehmen heute vor das Problem, dass Nationalstaaten eben nicht mehr in dem Maße wie früher jenen Ordnungsrahmen bereitstellen [...].“ (Suchanek, A. (2008b), S. 18, in der Quelle ebenfalls herv.).

510 Beschorner, T. (2013), S. 363.

${ }^{511}$ Pies, I. / Blome-Drees, F. (1993), S. 753, in der Quelle ebenfalls herv. Vgl. ähnlich auch Lingnau, V. (2011a), S. 39. Interessanterweise wiesen bereits HOMANN und BLOME-DREES in ihrem klassischen Lehrbuch darauf hin, ohne jedoch konsequent die zunehmende Bedeutung einer originären Unternehmensethik herauszustellen: „Die in der klassischen Konzeption an die Rahmenordnung delegierte Legitimationsverantwortung wirtschaftlichen Handelns fällt bei Defiziten in der Rahmenordnung an die Unternehmen zurück." (Homann, K. / BlomeDrees, F. (1992), S. 126, in der Quelle ebenfalls herv.).

512 Steinmann, H. (2004), S. 109, in der Quelle ebenfalls herv. 
von Gesetzen zukommen [... $]^{\text {“513 }}$ - zur Vermeidung einer ,negativen Grenzmoral“ ${ }^{514}$. Dies erscheint zwar aus der konzeptionellen Perspektive HOMANNS sicherlich methodisch stringent, unter der faktischen Realität einer begrenzten Steuerung durch formale (Rechts-)Normen zur (dominanten) Lösung ethischer Problemstellungen jedoch zu kurz gegriffen. Vielmehr scheint es sinnvoll, so zu argumentieren, dass basale, allgemeingültige Regeln in Rechtsnormen möglichst wirksam (und zukünftig sicherlich auch zunehmend im globalen Maßstab) abgedeckt werden (sollten), der forscherische Fokus allerdings unter den realen Bedingungen der modernen Wirtschaft mindestens ebenso stark auf die Mesoebene gelegt wird, andernfalls droht eine Vernachlässigung der real vielfach existierenden unternehmerischen Handlungsspielräume.

Ein weiterer klassischer Kritikpunkt wirft zudem die Frage auf, inwiefern sich moralisches Handeln rechnen muss. Wie aufgezeigt wurde, nutzt HOMANN häufig zwei Argumente, welche jedoch genauer differenziert werden sollten: So wird auf der einen Seite betont, moralisches Handeln müsse unter Nutzung des Eigeninteresses der Akteure motiviert werden bzw. solle sich „rechnen“ (Besserstellung), andererseits wird argumentiert, dieses Handeln dürfe nicht zu einer (dauerhaften) Schlechterstellung führen. Diese beiden Perspektiven fokussieren allerdings auf zwei unterschiedliche Sachverhalte. So erscheint es einsichtig, dass sich moralisches Handeln nicht immer rechnen $\mathrm{kann}^{515}$ (oder gar: muss! ${ }^{516}$ ). Dagegen kann sehr wohl formuliert werden, dass institutionelle Vorkehrungen so zu treffen sind, dass ein solches Handeln nicht zu einer systematischen, dauerhaften (gar existenziellen) Schlechterstellung bzw. Ausbeutbarkeit führt, was als eigentliche, zentrale Erkenntnis der Konzeption HomANNS aufgefasst werden kann. In diesem Sinne folgern auch AßLÁNDER und NUTZINGER: „Mit anderen Worten: es geht hier nicht darum, die Moral entsprechend den Vorteilserwartungen eigennutzorientierter Akteure auszugestalten, sondern darum, der Moral jene Steine aus dem Weg zu räumen, die ihre Befolgung erkennbar behindern oder zumindest erschweren." ${ }^{\text {(517 }}$

${ }^{513}$ Löhr, A. (2004), Sp. 1516. Vgl. auch Steinmann, H. (2008a), S. 341 sowie ebenfalls Ulrich, P. (1996), S. 20.

514 Steinmann, H. / Löhr, A. (1994a), S. 28.

515 In diesem Sinne betonen auch ROWLEY und BERMAN: „Only the most naïve (or blindly hopeful) among us will assume that poor (good) social behavior will always have negative (positive) financial implications.“ (Rowley, T. / Berman, S. (2000), S. 406).

516 Dies wäre die Auffassung einiger klassischer Ethikkonzeptionen, so z. B. auch bei KANT. ${ }^{517}$ Aßländer, M. S. / Nutzinger, H. G. (2010), S. 242. Dies entspricht der Auffassung ULRICHS von staatlichen Institutionen als „Rückenstützen“ (Ulrich, P. (2010), S. 98) des ethischen Handelns. Vgl. hierzu auch Thielemann, U. / Weibler, J. (2007a), S. 190. 
Die Konzeption HomanNs weist darüber hinaus auch noch weitere kritische Elemente bzw. Forschungslücken auf. So stellt sich, abgesehen von der primären Verortung von Moral in der Rahmenordnung, z. B. die Frage, wer genau für die Rahmensetzung legitimerweise Verantwortung übernehmen würde. Dies wäre klassischerweise der Staat mit dem korrespondierenden Gewaltmonopol zur Durchsetzung der Normen, welches jedoch in den Ausführungen HOMANNS recht kurz kommt. ${ }^{518}$ Zudem wäre indirekt auch letztlich die Bürgerschaft in Demokratien gefordert, partizipativ die Regeln einer solch verbesserten Wirtschaftsordnung mitzubestimmen, welches ebenfalls in HoMANNS Werk konzeptionell vernachlässigt wird. So konstatieren auch AßLÄNDER und NUTZINGER: „Woher sollen die höheren moralischen Vorstellungen der Gesellschaft kommen, wenn nicht von den Individuen, die sie konstituieren?"519 Des Weiteren stellt der Ansatz HomanNs auch eine tendenzielle Minimalethik dar, d. h. durch reine Regulierung werden i. d. R. lediglich die Mindeststandards im gleichermaßen modell-idealtypischen Grenzwettbewerb unter den Unternehmen erhöht, sofern nicht grundsätzlich staatliche Anreize bestimmte Handlungen per se fördern. Solch positive Förderungen sind jedoch stets aus fiskalischen Gründen limitiert, so dass auch hier letztlich kein (extrinsischer) Anreiz besteht, über das maximal geförderte Maß hinauszugehen.

Abschließend ist auch ein weiterer, bisher in der Literatur in Bezug auf HOMANNS Konzeption kaum diskutierter Aspekt hervorzuheben. So befasst sich die motivationstheoretische Grundlagenforschung schon des Längeren mit dem s. g. Crowding-Out-Effekt, d. h. der Verdrängung intrinsischer Motivation durch extrinsische Anreize. ${ }^{520}$ Ähnlich erscheint allerdings auch eine zu starke Regulierung über sanktionsbewährte Regeln problematisch, da diese den Akteuren den Eindruck vermitteln könnte, dass mit genuin intrinsischer, d. h. verinnerlichter Moral bzw. einem echten Ethos nicht mehr gerechnet wird ${ }^{521}$ und dieses

\footnotetext{
${ }^{518}$ Vgl. Heidenreich, F. (2012), S. 74-75.

${ }^{519}$ Aßländer, M. S. / Nutzinger, H. G. (2010), S. 243-244.

${ }^{520} \mathrm{Vgl}$. hierzu die zentralen Arbeiten in diesem Gebiet von Frey, B. S. (2012); Frey, B. S. / Jegen, R. (2001); Frey, B. S. / Oberholzer-Gee, F. (1997); Frey, B. S. (1994). Eine der bekanntesten, vielfach referenzierten Studien stammt in diesem Kontext von TITMUSS, welcher bereits in den 1970er Jahren Indizien für einen Crowding-Out-Effekt im Kontext von Blutspenden fand. Vgl. hierzu Titmuss, R. (1997). Grundlegend kann der Crowding-OutEffekt im Rahmen der Self-Determination Theory durch die Empfindung einer verminderten Selbstbestimmung erklärt werden. Vgl. hierzu Deci, E. L. / Ryan, R. M. (2008); Ryan, R. M. / Deci, E. L. (2002); Ryan, R. M. / Deci, E. L. (2000). Vgl. grundlegend auch Jost, P.-J. (2008), S. 499-502; North, K. / Güldenberg, S. (2008), S. 87.

${ }^{521}$ Vgl. hierzu auch Heidenreich, F. (2012), S. 73.
} 
demzufolge an handlungsleitender Bedeutung verlöre. Folglich könnte eine zu stark formalisierte Normenimplementierung über staatliche Sanktionswege, neben der potentiellen Wirksamkeitsproblematik an sich, auch das Phänomen eines moralischen Crowding-Out-Effekts evozieren.

Insgesamt kann es als das akademische Verdienst von HOMANN gelten, die Notwendigkeit der Berücksichtigung von Dilemmastrukturen im Wirtschaftskontext herausgearbeitet sowie das Augenmerk auf (staatlich) institutionalisierte Regeln gelegt zu haben, welche diese Dilemmata zum Teil überwinden helfen könnten. Wie HOMANN richtigerweise hinweist, können durch reine Idealverfolgung untergehende Unternehmen auch keinen gesellschaftlichen Bedürfnissen mehr dienen. Der dominante Fokus auf die Rahmenordnung erscheint jedoch (zumindest alleinig betrachtet) zu kurz zu greifen, externalisiert Verantwortung und führt zu einer zu starken Vernachlässigung der Mesoebene. Insbesondere im Kontext einer fehlenden Weltgesetzgebung erscheint der Ansatz daher aus einer Idealperspektive wichtig und durchaus konzeptionell wegweisend, in der Umsetzung aber bisher auf der globalen Ebene vielfach utopisch. Zudem bleibt schließlich auch der Fokus auf Rechtsnormen aus der Legitimitätsperspektive unternehmerischen Handelns problematisch, denn nicht alles, was legal ist, ist notwendigerweise auch bereits als legitim zu klassifizieren.

Die ausgeführten Kritikpunkte gelten dabei auch prinzipiell für die Schüler Homanns, welche seine Konzeption weiterentwickelt und in der Theoriebildung eigene Akzente gesetzt haben. Bei PIES konnte neben der Möglichkeit der institutionsökonomischen Ermittlung von Win-win-Situationen insbesondere die Hervorhebung der Notwendigkeit einer verstärkten didaktischen Beschäftigung mit ethischen Fragestellungen in wirtschaftswissenschaftlichen Veranstaltungen als genuine Aufgabe einer soliden Bildung zukünftiger Führungskräfte erachtet werden. ${ }^{522}$ Besonders interessant erscheint hierbei die Überlegung, Studierende der Wirtschaftswissenschaften durch Einbezug ethischer Lehrinhalte nicht (nur) zu besseren Menschen sondern (auch) zu besseren Managern auszubilden. Kritisch könnte in der Weiterentwicklung der Konzeption durch PIES allerdings der vorherrschende Top-down-Fokus in der ordonomischen Ethik sowie die Frage, inwiefern institutionell tatsächlich regelmäßig ethische Dilemmata in Winwin-Situationen transformiert werden können, erachtet werden. ${ }^{523}$ SUCHANEK

${ }^{522}$ Vgl. auch die Ausführungen bei Aaken, D. v. / Küpper, H.-U. / Schreck, P. (2011), S. 39 und S. 46-56, welche ebenso verstärkte Bildungsmaßnahmen im betriebswirtschaftlichen Curriculum als notwendig erachten, so dass die Studierenden nicht nur die konzeptionelle Breite ethischer Theorien kennen würden, sondern auch konkrete ethische Problemstellungen in der Praxis lösen könnten.

${ }^{523}$ Vgl. hierzu auch die Diskussion in Beschorner, T. / Kolmar, M. (2016), S. 447-451. 
befasst sich wiederum verstärkt mit unternehmensethischen Fragen, allerdings nach wie vor basierend auf den konzeptionellen Grundlagen HoMANNS. Zentrale Überlegung SUCHANEKS war hierbei, neben der Einführung eines praktischen Syllogismus sowie der Hervorhebung der Berücksichtigung des real vorherrschenden Regelverständnisses unter den Akteuren, auch die Fundierung unternehmensethischen Handelns als Investition. Während die ersten beiden Überlegungen SUCHANEKS als eher unproblematisch aufgefasst werden können, erscheint die Begründung von Ethik als „Investition“ in Vertrauen kritikwürdig. Obschon SUCHANEK stets überaus differenziert argumentiert ${ }^{524}$, erscheint der Begriff stark ökonomisch geprägt - welches sicherlich auch eine Anschlussfähigkeit an den klassischen wirtschaftswissenschaftlichen Diskurs intendiert. Damit steht im Kern der Argumentation letztlich ein kurzfristiger Gewinnverzicht, welcher sich später wiederum (für möglichst viele) ,auszahlen“ soll. Ethik muss sich folglich, wie auch in einer der Argumentationslinien bei HomANN, „lohnen“.525 So betont SUCHANEK: „Der Gewinnverzicht muss sich als Investition darstellen lassen." 526 Eine solche Argumentation im Sinne eines „Return on Ethics“ läuft jedoch stets Gefahr, nicht kategorienkonform zu argumentieren. So führt eine auf ökonomischen Größen aufbauende Unternehmensethik zu einem Kategorienfehler ${ }^{527}$, denn im Kern steht statt des eher strategischen Investitionsbegriffs besser der Begriff der Legitimität, dessen Sicherung im Rahmen eines normativen Managements als Rahmensetzung allen strategischen wie auch operativen Entscheidungen übergeordnet ist. Legitimität lässt sich so auch generell nicht sinnvoll in monetären Größen operationalisieren, denn die ,dabei anzuwendenden Kriterien entziehen sich weitgehend einer Diskontierung. Auf dieser Ebene stellt daher die Frage, ,ob sich das rechnet', einen Kategorienfehler dar, da Legitimität (ähnlich wie Vertrauen und Reziprozität) und (diskontierte) Zahlungsströme unterschiedlichen Kategorien angehören. Die Frage, um wieviel Euro sich die Legitimität denn nun durch eine Sozial- oder Umweltmaßnahme, deren Kosten bekannt sind, erhöhen werde (bzw. wieviel Euro an Legitimitätsverlust dadurch vermieden werden), ist offensichtlich unsinnig und nicht $\mathrm{zu}$ beantworten. “528 Zudem nimmt auch SUCHANEK an, dass eine solche „ethische Investition“ stets im

\footnotetext{
524 Vgl. z. B. Suchanek, A. (2015a), S. 51.

525 So betont auch klassisch ALBACH: „Opportunistisches Verhalten zerstört Vertrauen. Vertrauen aber ,lohnt sich“"“ (Albach, H. (2008), S. 7).

526 Suchanek, A. (2015a), S. 58, in der Quelle ebenfalls herv. Vgl. hierzu auch Osterloh, M. / Weibel, A. (2006), S. 17.

${ }^{527}$ Vgl. Lingnau, V. / Fuchs, F. (2021), S. 16; Ulrich, P. (2016a), S. 117.

${ }^{528}$ Lingnau, V. / Fuchs, F. (2021), S. 16.
} 
Sinne eines für alle Beteiligten gegenseitig wirksamen Vorteils gedacht werden sollte. ${ }^{529}$ Hierbei gilt letztlich eine ähnliche Kritik wie auch bei PIES, insofern zu problematisieren wäre, ob ethische Problemstellungen letztlich stets vollständig in eine Win-win-Situation aufzulösen sind. Schließlich kann auch kritisch aufgefasst werden, dass sich SUCHANEK in seinen konzeptionellen Überlegungen zwar auf die Mesoebene bezieht, die institutionellen Ausführungen zu wettbewerblich induzierten Dilemmasituationen aber nach wie vor von HOMANN makroethisch übernimmt. Damit erscheint gerade die naheliegende Frage, wie denn institutionelle Arrangements auf der Mesoebene gestaltet sein müssten, um erwünschtes Handeln zu unterstützen bzw. insbesondere auch unerwünschtes Handeln aus potentiellen Dilemmasituationen (z. B. ,wer kurzfristig betrügt, gewinnt“) zu demotivieren, ungeklärt. Dies wäre jedoch gerade für die Gestaltung unternehmerischer Anreizsysteme von zentraler Bedeutung, welches jedoch SUCHANEK nicht thematisiert. ${ }^{530}$ Daher wird sich die vorliegende Arbeit im Weiteren auch mit der möglichen Prävention adverser Effekte durch Anreizsysteme beschäftigen. ${ }^{531}$ Abschließend erfolgt auch bei SUCHANEK keine kritische Würdigung existierender Normen, mit welcher sich Unternehmensethik im Sinne normativer Führungsunterstützung verstärkt befassen müsste. ${ }^{532}$

Als dritte der klassischen Ethiken wurde schließlich die Konzeption ULRICHS erörtert, welcher sich auf die zwei aufgezeigten großen Strömungen der deutschsprachigen Wirtschaftsethik bezieht und diese kritisch rezipiert. Besonders hervorzuheben war in diesem Kontext neben der Bearbeitung aller drei Ebenen der Wirtschaftsethik aus mesoethischer Sicht die Herausstellung der Verantwortungsrelation von Unternehmen gegenüber ihren Stakeholdern (,,quasi öffentliche Institution") sowie die Bedeutung der Mitarbeit an einer verbesserten Rahmenordnung. Zentral war hierbei auch die kritische Grundlagenreflexion der bestehenden

${ }^{529}$ Vgl. nochmals Suchanek, A. (2015a), S. 59; Suchanek, A. (2015b), S. 17; Suchanek, A. (2015c), S. 60; Suchanek, A. (2008a), S. 4; Suchanek, A. (2007), S. 39; Suchanek, A. (2003), S. 9.

${ }^{530}$ Die fehlende Diskussion adverser Effekte durch klassische Anreizsysteme erscheint besonders verblüffend, bedenkt man, dass bereits HOMANN und BLOME-DREES auch auf der Organisationsebene (allerdings etwas unscharf) zwischen einer Individual- und Institutionenethik differenzierten. Vgl. Homann, K. / Blome-Drees, F. (1992), S. 118-120. Vgl. zu einer solchen Differenzierung auch Fenner, D. (2010), S. 8; Zimmerli, W. C. / Aßländer, M. S. (2010), S. 51 sowie die Überlegungen in Göbel, E. (2020), S. 116-117.

${ }^{531} \mathrm{Vgl}$. hierzu die Ausführungen in Abschnitt 4.6.2.3.

${ }^{532}$ Hierzu wird im Weiteren ein zweistufiger Ansatz entwickelt, welcher die instrumentelle Notwendigkeit einer Legitimitätssicherung auf Moralbasis mit einer kritisch-deskriptiven sowie potentiell ethisch-normativen Reflexion vereint. Vgl. hierzu auch die Ausführungen in Abschnitt 4.5.2. 
Normen, welche sich z. B. in gegenwärtigen Wirtschaftsrahmenordnungen niederschlagen, mit dem Ziel einer gemeinwohlorientierten bzw. lebensdienlichen Wirtschaft.

Auch an der Konzeption UlRICHS hat sich eine Fülle an kritischen Diskursen entzündet. So hebt z. B. BESCHORNER hervor, dass UlRICHS Ansatz vielfach aufgrund der stark theoretischen Ausrichtung und der angenommenen Idealkommunikationssituation gebildeter Wirtschaftsbürger kritisiert werde, da dieser zu wenig konkrete Anknüpfungspunkte für die Praxis biete. ${ }^{533}$ Auch GÖBEL reflektiert die idealbasierte Konzeption UlRICHS kritisch und betont, dass in dieser die ,faktische Auseinanderentwicklung von Ethik und Ökonomik zu wenig beachtet wird.“"534 Betrachtet man die Ausführungen UlRICHS in seinem Gesamtwerk, so ist in der Tat eine normativ-ideelle Vorprägung einer besseren Wirtschaftsordnung unverkennbar, welche seine Konzeption durchzieht. Dies wird insbesondere in der Basis seiner Ausführungen, dem aufgeklärten und gemeinwohlorientierten Wirtschaftsbürger, deutlich. So betont ULRICH paradigmatisch: „Als sich so verstehende Bürger sind sie [...] motiviert, auch ihr wirtschaftliches Vorteilsund Erfolgsstreben den Legitimitätsbedingungen der von ihnen mitgetragenen Gesellschaftsordnung zu unterstellen; sie wollen dann überhaupt keinen anderen privaten Erfolg als jenen, den sie im ,öffentlichen Vernunftgebrauch' (Kant, Rawls) unter freien Bürgern auch andern zubilligen und daher für sich selbst vertreten können. Genau dies ist der moralische Kern des republikanischen Wirtschaftsbürgerethos.“535 Auch an anderer Stelle hebt ULRICH im Kontext der Wirtschaftsbürger hervor, dass diese ,,von ihrem ganzen (ungespaltenen) Selbstverständnis her [..] überhaupt keinen anderen persönlichen Erfolg [wollen] als solchen, den sie im Lichte des Leitbilds der Gesellschaft, in der sie leben möchten und als deren Mitträger sie sich verstehen, vernünftigerweise als legitim vertreten können." ${ }^{536}$ Im Prinzip erscheint ULRICHS Konzeption damit überaus stark idealistisch bzw. normativ vorgeprägt. ${ }^{537}$ Allerdings existieren in ULRICHS Werk auch

${ }^{533}$ Vgl. Beschorner, T. (2006), S. 132. In diesem Sinne formuliert auch HEIDENREICH: „Anders als bei Homann oder Wieland erhalten Praktiker in Unternehmen wenig direkt umsetzbare Konzepte." (Heidenreich, F. (2012), S. 60).

534 Göbel, E. (2020), S. 94, in der Quelle fett herv.

535 Ulrich, P. (2007), S. 5, in der Quelle ebenfalls herv.

${ }^{536}$ Ulrich, P. (2004a), S. 69, in der Quelle ebenfalls herv.

${ }^{537}$ Wie in den wissenschaftstheoretischen Grundlagen bereits erörtert, kann kein wissenschaftliches System ohne normative Aussagen im Basisbereich existieren. In diesem Sinne betont auch ULRICH: „Irgendeine normativ orientierte ,preanalytic vision “ $[\ldots]$ ist wohlgemerkt jedem wissenschaftlichen Ansatz eigen, auch wenn er nicht auf normative (praktische) Handlungsorientierung, sondern allein auf theoretische Erklärung der vorgefundenen 
zentrale Passagen, in welchen eine normativ-fordernde und empirisch-deskriptive Argumentation ineinander übergehen. So hebt ULRICH beispielsweise hervor, dass Unternehmen ihre Existenz und den betrieblichen Erfolg sichern sollen (und wollen!) indem sie ,im Wettbewerb ausschliesslich mit gesellschaftlich legitimen und sinnvollen Strategien unternehmerischer Wertschöpfung "538 tätig werden. Hierbei wird ersichtlicherweise nicht klar, ob empirisch die faktischen Vorstellungen in einer Gesellschaft hinsichtlich Legitimität und Sinnhaftigkeit gemeint sind, oder vielmehr (vermutlich) eine normativ vorgeprägte Vorstellung einer philosophisch prädeterminierten idealen, aufgeklärten Gesellschaft - in welchem Falle jedoch wieder zu klären wäre, wer über diese „Vernünftigkeit“ letztlich zu urteilen hätte.

Hieran anknüpfend wurden auch die zentralen Begriffe der Konzeption ULRICHS einer kritischen Reflexion unterzogen. So konstatiert beispielsweise HEIDENREICH, dass ULRICHS Wirtschaftsethik eine Vielzahl von schillernden, aber letztlich unterbestimmten Begriffen aufweise, z. B. hinsichtlich der Begriffe der Lebensdienlichkeit oder der Vernünftigkeit. ${ }^{539}$ Der ontologische Status der Begriffe bleibt dabei vielfach unklar, gerade wenn ULRICH auf die Diskursethik zur Klärung dieser Terminologien Bezug nimmt, welche prozedural und damit (unter bestimmten Rahmenbedingungen) potentiell ergebnisoffen argumentiert. Zudem, so kritisieren STEINMANN und LÖHR, sei das diskursethische Element der Konzeption nicht praxisnah ausgelegt und impliziere, dass in der unternehmerischen Praxis ,im luftleeren Raum und ganz abstrakt über Betroffenheiten geredet werden könne." 540 Hinsichtlich der Praxisorientierung wurde ULRICH auch vielfach kritisiert, zu wenig mit einer Reflexion der realen ökonomischen

Wirklichkeit zielt.“ (Ulrich, P. (2004a), S. 63, in der Quelle ebenfalls herv.). Darüber hinaus betont UlRICH: ,Jede Vorstellung von einer ,lebensdienlichen“ Bewirtschaftung [..] im weitesten Sinn, jeder Begriff rationalen Wirtschaftens und jedes Konzept der Wirtschaftssubjekte impliziert also unausweichlich einen solchen normativen Vorgriff." (Ulrich, P. (2004a), S. 64). Dabei ist jedoch ersichtlich, dass seine Konzeption nicht nur über normative Hintergrundannahmen hinsichtlich einer besseren Wirtschaftsweise verfügt, sondern diese auch im Aussagenbereich einfordert. Denn so nimmt die Konzeption ULRICHS nicht nur an, sondern ,fordert also eine moderne Wirtschaftsbürgerethik von den Wirtschaftsakteuren [...] eine ethisch integrierte Erfolgsorientierung [.].“(Ulrich, P. (2004a), S. 69, in der Quelle nur z. T. herv.).

538 Ulrich, P. (2016a), S. 463.

${ }^{539}$ Vgl. Heidenreich, F. (2012), S. 60-61.

540 Steinmann, H. / Löhr, A. (1994b), S. 159, in der Quelle ebenfalls herv. Ganz im Sinne ihrer Konzeption fügen STEINMANN und LÖHR in diesem Kontext hinzu, ,daß das Gewinnprinzip eine historisch-strukturelle Vorgabe in der Wettbewerbswirtschaft [sei], die für ein Unternehmen nicht beliebig zur Disposition steh[e].“ (Steinmann, H. / Löhr, A. (1994b), S. 160). 
Praxis zu beginnen, sondern vielmehr diese angenommene „Praxis“ als Karikatur aus der neoklassischen Theorie zu übernehmen. In diesem Sinne betont auch BESCHORNER: „The crucial mistake of Integrative Business Ethics $[\ldots]$ is that it empiri-cally assumes ,business lifeworld“ as it is seen from orthodox economic and management perspectives - that is, value-free corporations and a value-free market system.“541 Damit kritisiere die Konzeption jedoch primär die ,ökonomische Theorie (z. B. in der Variante von Homann et al.), nicht jedoch [die] ökonomische Praxis. “542

Abschließend kann auch die intendierte Abgrenzung zu ,,instrumentalistischen" Theorien kritisch rezipiert werden. So postulieren THIELEMANN und UlRICH, dass integres Wirtschaften mit unternehmerischem Erfolg einhergehe, jedoch diese Erkenntnis nicht zur Motivation der Konzeption dienen soll: „Im Unterschied zum Instrumentalismus wird die Berücksichtigung legitimer Ansprüche nicht etwa davon abhängig gemacht, dass sich das für das Unternehmen auszahlt. Vielmehr ist das Unternehmen gerade darum nachhaltig erfolgreich, weil es ohne Wenn und Aber integer wirtschaftet." ${ }^{\text {"543 }}$ Unbeschadet der Feststellung, dass die Frage, ob sich integres Handeln „rechne“ in Gefahr eines Kategorienfehlers läuft, erscheint es zumindest fraglich, warum nicht, wenn eine integre Unternehmensführung das Unternehmen nachhaltig in seiner Existenz sichern könnte, diese Erkenntnis als praktisch relevante Begründung zur Verankerung ethischer Überlegungen in der Praxis herangezogen werden sollte. Diese Überlegung wird die Arbeit im Weiteren ebenfalls nochmals aufgreifen.

Zusammengefasst zeichnet sich die Konzeption UlRICHS durch mehrere zentrale Beiträge aus. Neben dem Einbezug aller drei Ebenen der Wirtschaftsethik kann auch die Auseinandersetzung mit unternehmensethischen Fragestellungen als bedeutsam erachtet werden. Darüber hinaus erscheint die Aufforderung, real existierende Werthaltungen kritisch zu überprüfen und nicht in einen unkritischen „Reflexionsstopp“ zu verfallen, von Relevanz und ist auch für die im Weiteren auszuarbeitende Konzeption einer zweistufigen Unternehmensethik, welche sowohl faktisch bestehende Werturteile einbezieht als auch zusätzlich einer kritischen Analyse unterziehen kann, von Bedeutung. Zudem kann der tendenziell eher praxisferne Ansatz ULRICHS kritisiert werden, welcher eher geringe Anhaltspunkte für konkrete betriebliche Entscheidungen offeriert und damit vielfach in der Idealsphäre einer anzustrebenden besseren Wirtschaftsrealität als kollektive zukünftige Änderungsanstrengung einer Gesellschaft verbleibt. Eine wichtige

541 Beschorner, T. (2006), S. 132.

542 Beschorner, T. (2013), S. 348.

543 Thielemann, U. / Ulrich, P. (2009), S. 41, in der Quelle ebenfalls herv. 
Ausnahme bildet jedoch die Aufforderung, sich mit den vorherrschenden Incentives in Unternehmen zu beschäftigen. So hebt ULRICH die Notwendigkeit einer Gestaltung ethisch reflektierter Anreizstrukturen in Unternehmen hervor, nach welchen ,ethisch verantwortungsvolles Handeln belohnt und rücksichtsloses, allein an persönlichen Bereicherungs- oder Karrierezielen orientiertes Verhalten demotiviert wird statt umgekehrt. “544 Dies erscheint auch für diese Arbeit von zentraler Bedeutung.

Abschließend ist noch zu klären, inwiefern, dem Arbeitstitel folgend, ULRICHS Konzeption tatsächlich eine Integration ethischer und ökonomischer Denkwelten vornimmt. Durch den vielfach betonten Vorrang bzw. des aristotelischen Primats der Ethik vor der Ökonomie scheint die Konzeption weniger eine Integration von Wirtschaft und Ethik zu repräsentieren. Vielmehr wird die Ökonomie durch die Ethik kritisch reflektiert bzw. ethisch geprägt, wodurch im strengen Sinne letztlich die Ethik - ähnlich wie bei STEINMANN und LOHR, allerdings fundamentaler vorgeschaltet wird.

\subsubsection{Reflexion der neueren Ansätze des deutschsprachigen Raums sowie der angelsächsischen Forschungstradition}

Neben den drei klassischen Strömungen werden im Weiteren nun auch zuerst einmal die neueren Entwicklungen der deutschsprachigen Wirtschaftsethikforschung jeweils einer kurzen, kritischen Reflexion unterzogen. Die Konzeption WIELANDS fokussiert dabei auf Unternehmen, welche als polylinguale soziale Systeme rekonstruiert wurden, wobei zur Stützung moralischer Handlungen die Notwendigkeit von Governancestrukturen, wie z. B. formale Institutionen aber auch freiwillige Selbstbindungsmaßnahmen, erarbeitet wurden. Grundgedanke war, dass moralbasierte Governanceregimes durch Vertrauensbildung zu einer Senkung der Transaktionskosten führen, wodurch dem Unternehmen langfristige Vorteile durch leichtere Interaktion mit seinen Stakeholdern zukommen können. In diesem Zusammenhang stellt WIELAND auch richtigerweise die zunehmende Bedeutung originärer unternehmerischer Verantwortung durch die Grenzen nationalstaatlicher Regulierung in der globalisierten Ökonomie heraus. ${ }^{545}$ Neben dem expliziten Fokus auf die Mesoebene und einer guten systemtheoretischen

${ }^{544}$ Ulrich, P. (2016a), S. 494, in der Quelle ebenfalls herv.

545 Vgl. Wieland, J. (2007), S. 24. 
Fundierung erscheint gerade die intendierte Praxisrelevanz ${ }^{546}$ vorzugswürdig, welches sich z. B. in Gestaltungsempfehlungen von Wertemanagementsystemen niederschlägt. So betont WIELAND, dass Ethik immer auch betriebswirtschaftlich relevant sein sollte, um in der Praxis Anwendung zu finden: „Ethik, die sich davor schützen und um ihrer selbst willen betrieben werden will, ist in den Unternehmen der Wirtschaft irrelevant. “" ${ }^{\text {"577 }}$ Interessant und innovativ erscheint darüber hinaus auch die aufgezeigte Überlegung WIELANDS, bei der Gestaltung von Anreizsystemen über die monetäre (oder i. w. S. materielle) Incentivierung hinaus, ebenfalls die Möglichkeit nichtmaterieller Anreize wie z. B. Anerkennung und Wertschätzung herauszuarbeiten ${ }^{548}$, welches sich für das Design zukünftiger, weniger dysfunktionaler Anreizsysteme als fruchtbar erweisen könnte. ${ }^{549}$

Als Kritikpunkt der Konzeption WIELANDS könnte der relativ formalistische Zugang zur Ethik diskutiert werden, welcher sich z. B. in der aufgezeigten Governanceformel niederschlägt. Zudem lässt die bisherige Governanceformel nur eine diskrete Ausprägung von Einflussfaktoren $(+1,0,-1) \mathrm{zu}$, so dass sich ein gradueller, mehr oder minder starker Einfluss von Governancestrukturen nicht abbilden lässt. Auch die Frage, inwiefern mehrere Governancestrategien, z. B. formale Institutionen, potentiell untereinander interagieren (z. B. Komplementarität, Konflikt usw.) und wie dies aggregativ im Modell abzubilden wäre, bleibt im Grundmodell WIELANDS bisher ausgeblendet. Des Weiteren fokussiert die Konzeption stark auf die Errichtung von Regelsystemen, welches die moralische Kompetenz von Individuen und Kollektiven z. T. unterschätzen sowie potentiell auch unterminieren könnte. ${ }^{550}$ In diesem Kontext weist BESCHORNER darauf hin, dass eine Ethik zudem auch stets die kritische Reflexion von Moral umfassen sollte ${ }^{551}$, weshalb dieser es ,bei dem derzeitigen Entwicklungsstand des

\footnotetext{
546 In diesem Sinne betont auch BESCHORNER, dass WIELAND einen dominanten Fokus auf „Anwendungs- und weniger auf Begründungsfragen“ (Beschorner, T. (2002), S. 125) legt.

${ }^{547}$ Wieland, J. (2007), S. 64. Vgl. ähnlich auch die Ausführungen bei Wieland, J. (2001b): ,[A]n ethics that seeks to protect itself against this and wants to be undertaken for its own sake is irrelevant in the real-life firms of the economy." (S. 80).

${ }^{548}$ Vgl. hierzu nochmals Wieland, J. (2007), S. 64; Wieland, J. (2005b), S. 122; Wieland, J. (2004b), S. 15-16 und S. 20-21.

${ }^{549} \mathrm{Vgl}$. hierzu auch die Ausführungen in Abschnitt 4.6.2.3.

${ }^{550} \mathrm{Vgl}$. hierzu nochmals die vorige Kritik an der Konzeption HoMANNS in Bezug auf einen möglichen moralischen Crowding-Out-Effekt.

${ }^{551}$ WIELAND versteht jedoch die von ihm entwickelte Governanceethik explizit als deskriptiv und kontextbezogen (vgl. Wieland, J. (2007), S. 29). In diesem Sinne wäre auch, insbesondere hinsichtlich des in der Fachwissenschaft faktisch existierenden Begriffs der deskriptiven
} 
Ansatzes von Josef Wieland für angemessener [hält], von einer Governancemoral, nicht aber von einer Governanceethik zu sprechen." ${ }^{452}$ Abschließend scheint jedoch auch die Überlegung, Ethik als Mittel zur Transaktionskostensenkung zu erachten, problematisch. So läuft auch diese Argumentationslinie, welche mit rein ökonomischen Kalkülen operiert, ähnlich wie bei SUCHANEK, Gefahr, nicht kategorienkonform zu argumentieren.

Die Konzeption nach BESCHORNER und PFRIEM wiederum rückt das Konzept der Kultur als zentrale Einflussgröße des kollektiven wie individuellen Handelns in den Fokus, wobei gerade in einer globalisierten Wirtschaftswelt die Relevanz kultureller Kompetenzen als zentraler unternehmerischer Erfolgsfaktor herausgearbeitet wird. Diese Hervorhebung der Bedeutung von Kultur im Wirtschaftskontext wird in der Literatur auch durch andere Konzeptionen aufgegriffen - wenngleich nicht im gleichen konzeptionellen, kulturwissenschaftlichen Detailgrad erörtert. So scheint die organisationale Kultur ebenso aus unternehmensethischer Perspektive nach STEINMANN und LÖHR relevant: „Immer wichtiger wird für eine erfolgreiche Handlungskoordination die sog. ,Unternehmenskultur “" "553 Gleichsam betont auch SUCHANEK im Rahmen seiner Konzeption die Relevanz von Unternehmenskultur: „Die formellen Institutionen allein vermögen jedoch nicht, alle anstehenden Abstimmungsprozesse - und die dabei auftretenden Dilemmastrukturen mit ihren Informations- und Anreizproblemen - zu regeln. Stets entwickeln sich auch informelle Regeln einschließlich gemeinsamer Denk-, Wahrnehmungs- und Verhaltensmuster der Mitarbeiter eines Unternehmens, die als Unternehmenskultur bezeichnet werden." ${ }^{554}$ Schließlich befasste sich auch KOSLOWSKI mit der Bedeutung von Kultur zur praktischen Umsetzung unternehmensethischer Überlegungen: „Das Unternehmen muss Unternehmenskultur entfalten, das heißt eine vor seinen Mitgliedern vertretbare Deutung seiner Aufgabe und Arbeitsweise und eine rechtfertigbare Lebensund Arbeitsordnung für die Teilnehmer am Unternehmensprozeß schaffen. “555 Die Konzeption der kulturalistischen Ethik ist allerdings in der Literatur bisher - womöglich aufgrund ihres jüngeren Entstehungsdatums - nur an wenigen

Ethik zu klären, ob - bereits rein terminologisch - nicht ein (ggf. sogar: rein!) empirisch ausgerichteter Ansatz auch als Ethik gelten kann.

552 Beschorner, T. (2010), S. 118-119. Vgl. hierzu auch Beschorner, T. (2002), S. 130-131 sowie den Überblick bei Heidenreich, F. (2012), S. 85-88.

553 Steinmann, H. / Löhr, A. (2002b), S. 96, in der Quelle ebenfalls herv.

554 Suchanek, A. (2007), S. 120, in der Quelle fett herv.

${ }^{555}$ Koslowski, P. (1989), S. 78. 
Stellen kritisch rezipiert worden. ${ }^{556}$ Als Kritikpunkt könnte hier z. B. der nach wie vor stark auf einer kulturwissenschaftlichen Grundlagenebene geführte Diskurs, welcher noch stärker konkret die praktischen Implikationen von Kultur in Unternehmen ausarbeiten sollte, hervorgehoben werden. So erscheint gerade das schillernde Konzept der Kultur hinsichtlich des Erfolges einer Verankerung bzw. Institutionalisierung ethischer Leitlinien in Unternehmen bedeutsam. In diesem Sinne gilt nach wie vor das klassische Zitat von HAAS: „[Y]ou can't force ethical conduct into an organization. Ethics is a function of the collective attitudes of our people." ${ }^{\text {557 }}$ Die konkreten kulturellen Randbedingungen, unter welchen ethische Überlegungen fruchtbar organisational institutionalisiert werden könnten, erscheinen somit für die zukünftige Forschung von besonderer Relevanz. Zusammenfassend könnte damit kritisch hervorgehoben werden, dass der Ansatz noch keine spezifische unternehmensethische Prägung aufweist, sondern eher recht allgemein das Konstrukt „Kultur“ in den Fokus der Betrachtung rückt, welches jedoch auf allen Ebenen der Gesellschaft handlungswirksam erscheint. Auf diese Weise könnte der Ansatz von BESCHORNER und PFRIEM auch als „Unternehmenskulturethik" bezeichnet werden, welche allerdings noch nicht zwingend eine eigenständige, allgemeine Unternehmensethikkonzeption darstellt.

Abschließend wurde auch mit KÜPPERS analytischer Ethik eine zentrale neue Entwicklung im Rahmen der deutschsprachigen Unternehmensethikforschung erörtert. Als besonders bedeutsam kann hier, neben einer expliziten und umfangreichen wissenschaftstheoretischen Fundierung, der Fokus auf die Analyse und Entscheidungsunterstützung realer ethischer Problemstellungen erachtet werden. Darüber hinaus erscheint auch die Überlegung, normative Aussagen mit Rückgriff auf grundlegendere, allgemein geteilte Werte fruchtbar und eröffnet z. B. einen Anknüpfungspunkt an die Forschungsergebnisse von KUNG im Rahmen des Weltethos. Als kritisch könnte in der Konzeption KÜPPERS der relativ starke Fokus auf individuelle Werte herausgestellt werden ${ }^{558}$, da hierbei der Einfluss kollektiver Werte tendenziell vernachlässigt wird. Zudem operiert KUPPER hinsichtlich der Terminologien nicht immer vollständig konsistent und gebraucht z. B. zentrale Begriffe wie Werte und Ziele relativ undifferenziert. ${ }^{559}$ Die Konzeption des analytischen Frameworks erscheint dabei überaus wegweisend zur Verbindung konzeptioneller und praktischer Relevanz, greift aber mit dem Fokus auf eher unspezifische „ethische Probleme“ noch zu kurz. So erscheint auch

\footnotetext{
556 Eine Ausnahme stellt hier z. B. der Beitrag von Pies, I. (2016b) dar.

${ }^{557}$ Haas, R. D. (1998), S. 216, in Bezug auf das Unternehmen Levi Strauss \& Co.

558 Vgl. nochmals z. B. Küpper, H.-U. (2011), S. 172-173.

${ }^{559}$ Vgl. z. B. Küpper, H.-U. (2011), S. 172.
} 
die Restriktion auf individuelle (Mitarbeiter-)Werte und Konfliktpotentiale durch unterschiedliche Werthaltungen als konzeptionell zu eng und fokussiert lediglich auf die internen Stakeholder. Fruchtbarer erscheint dagegen, aus unternehmensethischer Perspektive mit sämtlichen Stakeholdern des Unternehmens zu beginnen und deren Ansprüche auf normativer Ebene des Managements einer Legitimitätsprüfung zu unterziehen, um fundiert zu begründen, welche Stakeholderansprüche in welchem Ausmaß in das organisationale Zielsystem aufgenommen werden. ${ }^{560}$

Schließlich diskutierte die Arbeit auch die angelsächsischen Beiträge der Business Ethics. Hierbei fiel die starke Praxisorientierung der Beiträge auf, welches vor dem Hintergrund des dominierenden Wissenschaftsparadigmas des Pragmatismus zu erklären ist. In diesem Kontext ist insbesondere die Herausstellung der praktischen Bedeutung von wirtschaftsethischen Überlegungen zentral, welches sich z. B. in der Entwicklung dezidierter „Ethiktools“ zur normativen Entscheidungsunterstützung manifestiert. Dabei kann jedoch (aus Perspektive der deutschsprachigen Forschung) die teilweise unklare bzw. fehlende theoretische Fundierung der Beiträge kritisiert werden. Zudem erscheint, gerade vor dem Hintergrund der zunehmenden Entscheidungsmacht von Kollektiven, der dominante Fokus der angelsächsischen Tradition auf die Individualebene, welcher originäre mesoethische Überlegungen vielfach ausklammert, problematisch. Dennoch kann abschließend wiederum - analog zu den Ausführungen bei PIES - die Betonung der didaktischen Vermittlung wirtschaftsethischer Reflexionsund Argumentationskompetenzen an zukünftige Führungskräfte im Rahmen des wirtschaftswissenschaftlichen Studiums als Stärke dieser Forschungs- und Lehrtradition hervorgehoben werden. 561

\subsection{Schlussfolgerung - Oder: Die Relevanz und Motivation einer neuen, genuinen Mesoethik}

Nachdem die bisherigen Ausführungen die zentralen Konzeptionen der Wirtschaftsethik einer kritischen Würdigung unterzogen haben, wird im Weiteren nochmals ein integrativer Gesamtblick auf den Forschungsstand herausgearbeitet und zudem die Notwendigkeit einer neuartigen Konzeption der Unternehmensethik aufgezeigt. Eine zentrale Frage, welche die kritische Würdigung der

\footnotetext{
${ }^{560}$ Diese fundamentalen Überlegungen wird die Arbeit in Abschnittt 4.5 wieder aufgreifen und detailliert ausarbeiten.

${ }^{561} \mathrm{Da}$ die angelsächsische Forschungstradition, wie aufgezeigt wurde, originär konzeptionellen Überlegungen einen eher geringen Stellenwert zuweist, sei hier auf eine nochmalige detailliertere Diskussion der Grundlagentexte der wesentlichen Autoren verzichtet.
} 
bisherigen Konzeptionen aufwerfen könnte, wäre, inwiefern die diskutierten Kritikpunkte in einem absoluten Sinne als konzeptionelle Schwächen aufzufassen wären - wie dies gerade die häufig zwischen den wirtschaftsethischen Schulen intensiv geführten Kontroversen um „den“ richtigen Zugang zur Wirtschaftsethik nahelegen könnten. Dabei stellt sich die Frage, ob die verschiedenen Konzeptionen nicht weniger auf streng gegenseitig inkompatiblen Basisannahmen beruhen, sondern vielmehr lediglich einen potentiellen Zugang zu Fragen der Wirtschaftsethik repräsentieren. ${ }^{562}$ Wirft man einen genaueren Blick auf die Auseinandersetzung der einzelnen Schulen, so wird deutlich, dass viele kritische Rezeptionen auf eine undifferenzierte Zuspitzung von Argumentationslinien hinauslaufen, welches jedoch in einem Gesamtblick betrachtet nicht zwingend notwendig erscheint. Auch HomanN beklagt im deutschsprachigen Raum in diesem Sinne ,the development of numerous misunderstandings, reciprocal assumptions, and deep and misguided antagonisms“"563, welche prinzipiell unnötig seien. ${ }^{564}$ So vernachlässigen, wie diskutiert wurde, z. B. auch STEINMANN und LOHR nicht die Notwendigkeit, grundlegende Normen in der Rahmenordnung zu implementieren, fokussieren jedoch im Kontext unvollkommener Rahmenordnungen auf die Unternehmensethik, während sich HOMANN vornehmlich mit der Implementierung von Normen in der Wirtschaftsrahmenordnung beschäftigt, im Rahmen unvollkommener Rahmenordnungen jedoch auch die Relevanz originärer unternehmerischer Verantwortung herausstellt. Damit scheinen in der Tat die verschiedenen Schulen schlicht einen verschiedenen Fokus auf wirtschaftsethische Probleme zu nehmen, allen geht es im Kern jedoch um „mehr“ bzw. bewusstere Reflexion und Implementation von Ethik im wirtschaftlichen Handeln von Individuen wie auch Kollektiven und damit letztlich um genuin humanistische Zielsetzungen. Die verschiedenen Theoriestränge und ihre zentralen konzeptionellen Beiträge subsumiert nochmals die nachfolgende Abbildung 3.14.

Wie diskutiert wurde, können die zentralen Erkenntnisse der verschiedenen Wirtschaftsethiken auch für die Entwicklung der Unternehmensethikkonzeption im Rahmen dieser Arbeit wichtige Impulse liefern. Dabei erscheint gerade die im

562 Sie können damit nach POPPER im metaphorischen Sinne als „Scheinwerfer“ aufgefasst werden, mit dem die jeweilige Theorie schlichtweg einen spezifischen Realitätsausschnitt hervorhebt. Vgl. hierzu Popper, K. R. (2011), S. 466; Popper, K. R. (2002), S. 171; Popper, K. R. (1979), S. 346.

563 Homann, K. (2016), S. 37.

${ }^{564}$ In diesem Sinne äußert sich auch WIELAND nach einer kritischen Rezeption der Konzeption HOMANNS versöhnlich und betont, dass ,nicht der beträchtliche Grad an Komplementarität und Übereinstimmung zwischen den beiden Ansätzen übersehen werden [sollte].“ (Wieland, J. (2001a), S. 33). 


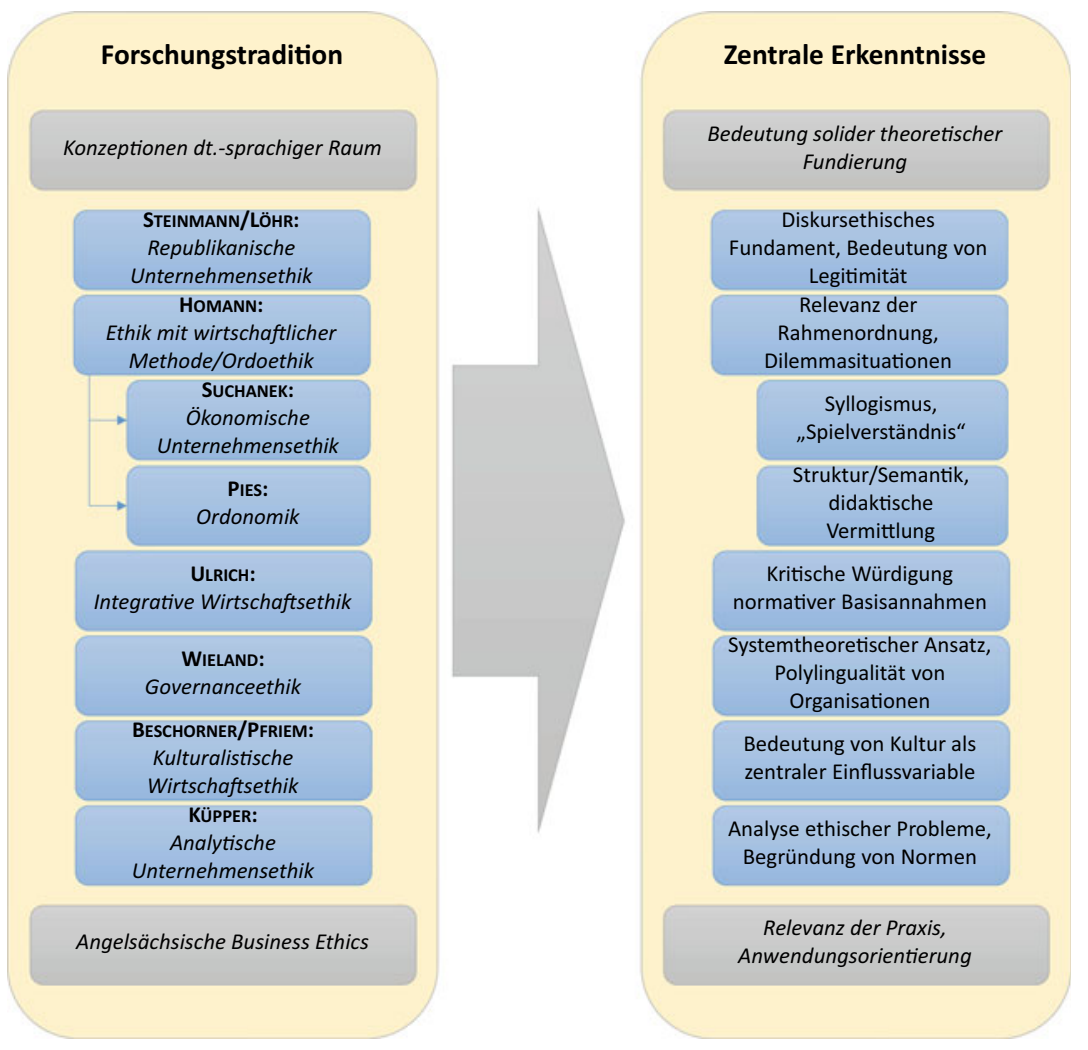

Abbildung 3.14 Zusammenfassung der zentralen wirtschaftsethischen Forschungstraditionen im deutschsprachigen und angelsächsischen Raum und ihre Erkenntnisse

deutschsprachigen Raum vertretene Forschung durch ihren Fokus auf solide theoretische Grundlagenarbeit zur Fundierung der zu entwickelnden Unternehmensethikkonzeption fruchtbar - so z. B. im Rahmen der Konzeption von STEINMANN und LÖHR der Fokus auf den unternehmerischen Legitimitätserhalt sowie das diskursive Fundament. Dagegen zeigt sich in der Herausarbeitung und Reflexion möglicher Dilemmastrukturen die zentrale Stärke der Schule um HomanN. In den verschiedenen Weiterentwicklungen des Ansatzes ist für die zu entwickelnde Konzeption auch die Überlegung SUCHANEKS interessant, Stakeholderansprüche strukturiert in einem Framework hinsichtlich ihrer möglichen Umsetzbarkeit zu 
reflektieren - obschon SUCHANEK wiederum in der ökonomischen Terminologie der Ethik als „Investition“ nicht gefolgt wird. Aus der Weiterentwicklung von PIES kann dabei insbesondere die Relevanz der didaktischen Vermittlung von Wissen und diskursiven Kompetenzen im wirtschaftsethischen Kontext hervorgehoben werden. ULRICHS Konzeption bietet darüber hinausgehend durch ihre kritische Reflexion faktisch herrschender Normen einen wichtigen Anstoß zu einer zweistufigen Reflexion der Ethik, welche neben einer rein faktisch-moralischen Ebene auch im zweiten Schritt eine kritisch-deskriptiv fundierte sowie eine potentiell ethisch-normative Reflexionsbasis offeriert. WIELANDS Ansatz wiederum erscheint besonders durch seinen systemtheoretischen Zugang, wenngleich nur auf die soziale Dimension des Unternehmens beschränkt, fruchtbar für die zu entwickelnde Unternehmensethikkonzeption. Auch die Hervorhebung, dass Unternehmen nicht nur ökonomisch, sondern auch moralisch überzeugend kommunizieren und handeln müssen, erscheint - wie die Betonung der Erweiterung von Anreizsystemen in der moralischen Dimension - relevant. Die kulturwissenschaftlich geprägte Ethik von BESCHORNER und PFRIEM hebt dagegen mit dem Konstrukt der Kultur eine zentrale Einflussgröße auf individuelles wie kollektives Handeln hervor. Diese Überlegungen erscheinen gerade im Sinne einer erfolgreichen Institutionalisierung ethischer Überlegungen in Unternehmen bedeutsam. Aber auch der Ansatz von KÜPPER kann mit seinem analytischen Fokus zur normativen Managementunterstützung, speziell durch sein differenziertes wissenschaftstheoretisches Fundament, als zentraler Impulsgeber für die Gestaltung der Unternehmensethikkonzeption in dieser Arbeit erachtet werden.

Betrachtet man den bisherigen Forschungsstand und die kritische Rezeption der Forschung, so zeigt sich bei aller Fülle konzeptioneller Zugänge zum Forschungsfeld der Wirtschaftsethik allerdings, dass insbesondere hinsichtlich der Begründung von Unternehmensethik noch keine überzeugende Konzeption gefunden wurde. So verbleiben Ansätze, welche spezifisch auf die Unternehmensebene fokussieren, bisher stark in der Domäne der reinen Appelle (STEINMANN und LOHR), skizzieren lediglich eine idealisierte, lebensdienlichere Wirtschaftswelt aus normativer Perspektive (ULRICH) oder folgen einer dominant ökonomischen Argumentation, welche Unternehmensethik als Investition zur gegenseitigen Besserstellung (SUCHANEK) oder zur Senkung von Transaktionskosten (WIELAND) auffasst. In dieser Zusammenschau zeigt sich, dass bis heute keine Konzeption existiert, welche als originäre Mesoethik die intrinsische Bedeutung von praktisch relevanter Ethik im Sinne einer Verantwortungsübernahme im Unternehmenskontext überzeugend herauszuarbeiten vermag, ohne wiederum auf traditionelle ökonomische Argumentationslinien oder reine Appelle zurückzufallen. Die Relevanz dieser aktuell offenen Forschungsfrage betont auch NEUHÄUSER: „In der 
Praxis muss allerdings einiges geleistet werden, damit Unternehmen tatsächlich in die Lage kommen, mehr Verantwortung zu tragen. Sie mögen dazu zwar grundsätzlich fähig sein, sind aber offensichtlich kaum motiviert, ethisch zu handeln. Woher aber soll diese Motivation kommen?" 565 Genau dieser originär konzeptionellen Begründungsfrage der Unternehmensethik wird sich diese Arbeit dezidiert annehmen, wobei, wie im Weiteren noch gezeigt wird, hierzu ein neuartiger systemtheoretischer Zugang fruchtbar erscheint, um eine konzeptionelle Begründung intrinsischer Relevanz der Unternehmensethik zu erarbeiten.

Hierbei soll die Konzeption jedoch nicht nur theoretisch wohlfundiert sein. Sie intendiert damit nicht nur ein vertieftes theoretisches Verständnis wirtschaftsethischer Zusammenhänge auf der Mesoebene, sondern ist zudem auch durch die praxisorientierten Überlegungen der angelsächsischen Business Ethics beeinflusst und soll in diesem Sinne auch für die Praxis wertvolle Hilfestellungen offerieren. Die Arbeit folgt damit aus wissenschaftstheoretischer Perspektive einem Mittelweg, der zwischen einer reinen Grundlagenforschung einerseits und einer vornehmlich anwendungsgetriebenen Forschung andererseits eingeordnet werden kann, welches STOKES auch als „Use-inspired basic research“ (anwendungsgetriebene Grundlagenforschung) bezeichnet. Zentral ist folglich zum einen die solide theoretische Fundierung der Konzeption, zum anderen aber auch deren Praxisrelevanz. Ziel ist demzufolge, dass die Konzeption ein detailliert ausgearbeitetes theoretisches Fundament aufweist, intendiert dieses jedoch nicht nur der reinen Erkenntnis wegen, sondern im Sinne einer reflektierten praktisch-normativen BWL auch zur fundierten, anwendungsorientierten Lösung realer unternehmerischer Problemstellungen im normativen Kontext. Diese Überlegungen fasst die nachfolgende Abbildung 3.15 nochmals zusammen.

Die Relevanz solcher Überlegungen stellen auch STEINMANN und LOHR heraus. So sollte in deren Perspektive „das vorzuschlagende Konzept von Unternehmensethik aber auch eine - von der mit Ethik befaßten ,praktischen Philosophie" häufig vergeblich angemahnte - Praxisrelevanz besitzen."566 Es geht hierbei folglich um die Entwicklung einer fundierten Konzeption mit Bezug zur „,betrieblichen und allgemein gesellschaftlichen Praxis. “567 Schließlich betont ebenso BESCHORNER: „In diesem an dieser Stelle lediglich andeutbaren Sinne

\footnotetext{
565 Neuhäuser, C. (2011a), S. 181. Hierbei wird die Arbeit im Nachfolgenden zeigen, dass eine solche Motivation in der überlebenskritischen Sicherung der gesellschaftlichen Legitimität als normativer Vorsteuerungsgröße aufgefunden werden kann.

566 Steinmann, H. / Löhr, A. (1988), S. 301, in der Quelle ebenfalls herv.

567 Steinmann, H. / Löhr, A. (2015), S. 282.
} 
Beachtung der praktischen Nutzbarkeit?

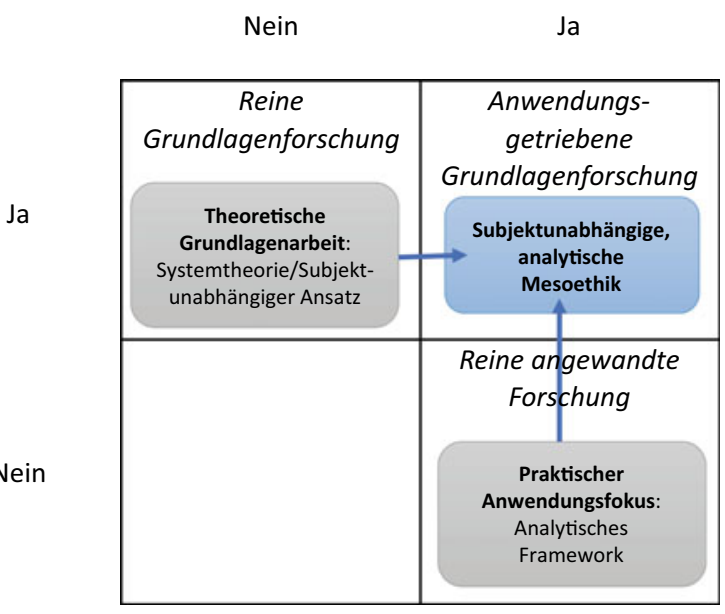

Abbildung 3.15 Einordnung der zu entwickelnden unternehmensethischen Konzeption in das Klassifikationsschema der Ausprägungen wissenschaftlicher Forschung nach STOKES 568

würde ich mir für die Wirtschaftsethik in den kommenden Jahren eine stärkere Brückenbildung zwischen begründungs- und anwendungstheoretischen Frage [sic!] wünschen. Ich sehe sie weder in der deutschsprachigen Diskussion noch international noch in den eigenen Arbeiten hinreichend bearbeitet. “569

Fasst man damit abschließend die vorausgegangenen Ausführungen zusammen, so sind mehrere offene Forschungsfragen $\mathrm{zu}$ konstatieren, welche bisher in der Literatur noch nicht eingängig bzw. im Zusammenhang erforscht wurden. So ist erstens die Frage zu klären, wer unter den Bedingungen der heutigen, modernen Wirtschaftswelt als (primärer) Träger von Verantwortung aufzufassen wäre. In klassisch-ethischer Betrachtung sind dies vornehmlich menschliche Individuen, wobei zur Begründung der Verantwortungsrelation ein bestimmter aufklärerischer Subjektbegriff zugrunde gelegt wird. Dieser wird jedoch seit einiger Zeit mit einer Vielzahl von Gründen selbst in der philosophischen Literatur zunehmend kritisch rezipiert. Darüber hinaus werden mit dem Wandel

568 In Anlehnung an Stokes, D. E. (1997), S. 73.

${ }^{569}$ Beschorner, T. (2013), S. 367. 
hin zu einer globalisierten Wissensgesellschaft weitere Restriktionen einer solchen klassischen Individualattribution von Verantwortung deutlich. In diesem Zusammenhang ist nun allerdings kritisch festzustellen, dass sich die bisherigen Konzeptionen mit den Implikationen der aufgezeigten gesellschaftlichen Transformation kaum befasst haben, so dass in diesem Kontext eine gewisse konzeptionelle „Sprachlosigkeit“ zu konstatieren ist. Dabei stoßen gerade auch die ordoethischen Konzeptionen durch die abnehmende Regulierungsmacht der Einzelstaaten zunehmend an ihre argumentativen Grenzen. Da bisher noch keine wirtschaftsethische Konzeption existiert, welche die genannten gesellschaftlichen Veränderungen hinreichend zu berücksichtigen vermag, sind im Weiteren die folgenden Aspekte näher zu untersuchen:

- Welche Grenzen ergeben sich aus den Limitationen einer klassischen subjektphilosophischen Verantwortungszuschreibung auf Individual- bzw. Mikroebene aus dem philosophischen Diskurs sowie insbesondere auch vor dem Hintergrund des Faktums einer globalisierten Wissensgesellschaft?

- Warum kann eine alternative Verortung der Verantwortungsinstitutionalisierung auf vornehmlich der gesellschaftlichen Ebene bzw. Makroebene, z. B. durch staatliche Regulierung, ebenfalls als nicht hinreichend aufgefasst werden?

- Könnten dann ggf. alternativ Unternehmen als zentrale Verantwortungsträger auf der Mesoebene aufgefasst werden?

Falls gezeigt werden kann, dass Unternehmen in zunehmendem Maße selbst Verantwortung übernehmen sollten, wäre jedoch zweitens die bisher ungeklärte Frage zu beantworten, wie eine solche Verantwortungsrelation überzeugend und kategorienkonform begründet werden kann. Bisherige Ansätze greifen dabei einerseits auf Moralappelle zurück, wie bspw. bei STEINMANN und LÖHR oder ULRICH, als Unternehmen mehr Verantwortung zu übernehmen, oder rekurrieren andererseits auf vornehmlich staatliche Interventionen auf der Makroebene, so die Schule von Homann. In diesem Zusammenhang ist hinsichtlich der „Motivation“ der Unternehmen folglich der nachfolgende Aspekt zu klären:

- Wie kann die Relevanz einer Übernahme von unternehmerischer Verantwortung begründet werden, ohne

a) diese rein appellativ einzufordern (,Einsicht“ bzw. altruistische Präferenzen) oder andernfalls, 
b) auf traditionelle ökonomisch-basierte Argumentationsmuster im Sinne eines „Return on Ethics“ zurückzufallen, womit jedoch ein Kategorienfehler droht?

Aus konzeptioneller Perspektive ist in diesem Zusammenhang zudem drittens festzustellen, dass bisher kaum eine vertiefte Auseinandersetzung hinsichtlich der Begründung einer genuinen Mesoethik stattgefunden hat. So handelt es sich bei den meisten existierenden Ansätzen entweder um die Problematisierung ethischer Fragen in Unternehmen oder um eher aggregative Individualethiken. ${ }^{570}$ Ein grundsätzliches Problem der bisherigen Konzeptionen dürfte auch sein, dass diese sich bisher kaum um ein vertieftes Verständnis ihres Erfahrungsobjektes, das Unternehmen (bzw. verallgemeinert: der Betrieb), bemüht haben bzw. implizit auf älteren, heute weniger zweckmäßigen Konzeptionen aufbauen und damit folgerichtig auch keine genuine Ethik auf einer eigenständigen betrieblichen bzw. unternehmerischen Ebene entwickelt werden konnte. Betriebe, sowie im Spezifischen Unternehmen, stellen jedoch, wie noch detailliert gezeigt wird, nicht nur Ansammlungen von Menschen und technischen Mitteln dar, sondern weisen als dauerhaftes sozio-technisches System einen eigenen Status auf, welchen es konzeptionell zu berücksichtigen gilt. Daraus folgt dann zum einen die Notwendigkeit einer eigenständigen konzeptionellen Fundierung des Unternehmens sowie hierauf aufbauend zum anderen die Möglichkeit einer eigenständigen ethischen Fundierung, wobei gerade nicht auf einen Begründungstransfer von problematischen Subjekteigenschaften auf die Mesoebene zurückgegriffen werden soll. Es wären damit spezifisch die folgenden Fragen zu klären:

- Wie kann eine Ethikkonzeption der Mesoethik fundiert werden, welche

a) eine originäre Mesoethik darstellt, also auf eine Entität rekurriert, welche „mehr ist“ als die Summe ihrer Komponenten sowie

b) nicht auf dem klassischen Subjektparadigma basiert?

- Wie können vor diesem Hintergrund und in Beachtung der Fragestellungen zuvor die Erkenntnisse der neueren Systemtheorie für eine alternative Begründung der intrinsischen Relevanz von Unternehmensethik fruchtbar gemacht werden?

\footnotetext{
${ }^{570}$ Am ehesten konzipiert noch WIELAND eine systemische Ethik, begründet diese jedoch mit dem ökonomischen Argument einer Transaktionskostensenkung, d. h. primär auf operativer Ebene. Vgl. hierzu nochmals die Ausführungen in Abschnittt 3.4.5.1.
} 
Schließlich kann viertens noch aus methodischer Perspektive festgestellt werden, dass ein gegenseitiger Austausch der deutschsprachigen, relativ theoriefokussierten sowie angelsächsischen, eher pragmatisch wie praktisch orientierten, Forschungstraditionen bisher nur in geringem Maße stattgefunden hat, so dass diese Richtungen bislang relativ unverbunden koexistieren. Es wäre folglich zu klären, inwiefern eine neue Konzeption geschaffen werden kann, welche beide Forschungsrichtungen integriert. Demnach stellt sich die Frage:

- Wie kann eine Konzeption der Unternehmensethik entwickelt werden, welche eine Brücke zwischen solider theoretischer Grundlagenforschung und zugleich praktischer Relevanz und Umsetzbarkeit der Erkenntnisse zu schlagen vermag?

Mit diesen aufgezeigten, offenen Forschungsfragen wird sich die Arbeit im folgenden vierten Kapitel beschäftigen.

Open Access Dieses Kapitel wird unter der Creative Commons Namensnennung 4.0 International Lizenz (http://creativecommons.org/licenses/by/4.0/deed.de) veröffentlicht, welche die Nutzung, Vervielfältigung, Bearbeitung, Verbreitung und Wiedergabe in jeglichem Medium und Format erlaubt, sofern Sie den/die ursprünglichen Autor(en) und die Quelle ordnungsgemäß nennen, einen Link zur Creative Commons Lizenz beifügen und angeben, ob Änderungen vorgenommen wurden.

Die in diesem Kapitel enthaltenen Bilder und sonstiges Drittmaterial unterliegen ebenfalls der genannten Creative Commons Lizenz, sofern sich aus der Abbildungslegende nichts anderes ergibt. Sofern das betreffende Material nicht unter der genannten Creative Commons Lizenz steht und die betreffende Handlung nicht nach gesetzlichen Vorschriften erlaubt ist, ist für die oben aufgeführten Weiterverwendungen des Materials die Einwilligung des jeweiligen Rechteinhabers einzuholen.

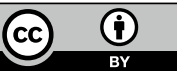

